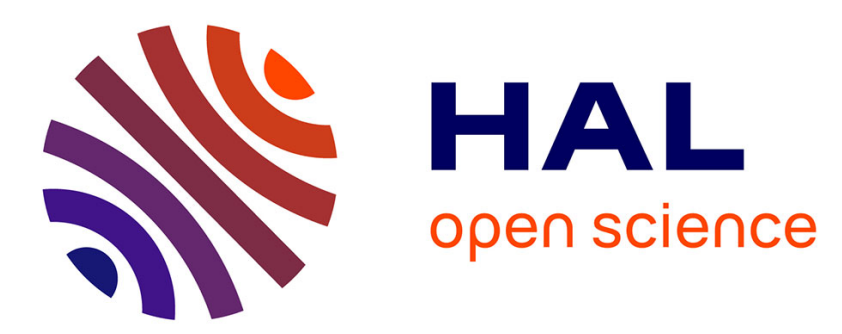

\title{
The 3D time-dependent Oseen system: link between L p -integrability in time and pointwise decay in space of the velocity
}

Paul Deuring

\section{To cite this version:}

Paul Deuring. The 3D time-dependent Oseen system: link between L p -integrability in time and pointwise decay in space of the velocity. 2020. hal-02465649v2

\section{HAL Id: hal-02465649 \\ https://hal.science/hal-02465649v2}

Preprint submitted on 17 Mar 2020

HAL is a multi-disciplinary open access archive for the deposit and dissemination of scientific research documents, whether they are published or not. The documents may come from teaching and research institutions in France or abroad, or from public or private research centers.
L'archive ouverte pluridisciplinaire HAL, est destinée au dépôt et à la diffusion de documents scientifiques de niveau recherche, publiés ou non, émanant des établissements d'enseignement et de recherche français ou étrangers, des laboratoires publics ou privés. 


\title{
The 3D time-dependent Oseen system: link between $L^{p}$-integrability in time and pointwise decay in space of the velocity.
}

\author{
Paul Deuring
}

Université du Littoral Côte d'Opale, Laboratoire de Mathématiques Pures et Appliquées Joseph Liouville, F-62228 Calais, France.

\begin{abstract}
A representation formula without pressure term is derived for regular solutions to the 3D time-dependent Oseen system in exterior Lipschitz domains. This formula is valid even if no boundary conditions are imposed. It is used in order to exhibit how the velocity decays pointwise in space. It turns out that the rate of this decay depends on $L^{p}$-integrability in time of the velocity. In addition, this work is the basis for successor papers dealing with spatial decay of $L^{q}$-weak solutions and mild solutions to the time-dependent Oseen system, and with $L^{2}$-strong solutions to the stability problem related to the Navier-Stokes system with Oseen term.
\end{abstract}

AMS subject classifications. 35Q30, 65N30, 76D05.

Key words. Oseen system, integral representation, decay.

\section{Introduction}

We consider the time-dependent Oseen system

$$
\partial_{t} u-\Delta_{x} u+\tau \partial_{x_{1}} u+\nabla_{x} \pi=f, \operatorname{div}_{x} u=0 \quad \text { in } \bar{\Omega}^{c} \times\left(0, T_{0}\right),
$$

where $\bar{\Omega}^{c}:=\mathbb{R}^{3} \backslash \bar{\Omega}$ denotes an exterior domain, with $\Omega \subset \mathbb{R}^{3}$ being an open bounded set with Lipschitz boundary. This set is given, as are the quantities $T_{0} \in(0, \infty]$ and $\tau \in(0, \infty)$, as well as the function $f: \bar{\Omega}^{c} \times\left(0, T_{0}\right) \mapsto \mathbb{R}$. The unknowns are the functions $u: \bar{\Omega}^{c} \times\left(0, T_{0}\right) \mapsto \mathbb{R}^{3}$ and $\pi: \bar{\Omega}^{c} \times\left(0, T_{0}\right) \mapsto \mathbb{R}$. The Oseen system is a linearization of the Navier-Stokes system with Oseen term

$$
\partial_{t} u-\Delta_{x} u+\tau \partial_{x_{1}} u+\tau\left(u \cdot \nabla_{x}\right) u+\nabla_{x} \pi=f, \operatorname{div}_{x} u=0 \quad \text { in } \bar{\Omega}^{c} \times\left(0, T_{0}\right),
$$

which models the flow of a viscous incompressible fluid around a rigid body moving with constant velocity and without rotation. In this model, the reference frame used to describe the flow is supposed to adhere to the rigid body. The functions $u$ and $\pi$ correspond respectively to the velocity and the pressure field of the fluid, the function $f$ stands for an exterior force acting on the fluid, and $\tau$ may be interpreted as the Reynolds number of the fluid.

In the work at hand, we want to exhibit pointwise decay in space of the velocity part $u$ of a solution to (1.1). More precisely, if $u$ is the velocity part of a regular solution to (1.1) as specified in Corollary 5.2 and Theorem 6.1, and if $|f(x, t)|$ and $|u(x, 0)|$ decrease sufficiently fast for $|x| \rightarrow \infty$, then the estimate

$$
\left|\partial_{x}^{\alpha} u(x, t)\right| \leq \mathfrak{C}\left[(|x| \nu(x))^{-(3+|\alpha|) / 2+1 /\left(2 \min \left\{\sigma_{1}^{\prime}, \sigma_{2}^{\prime}, \sigma_{3}^{\prime}\right\}\right)}+|x|^{-(\gamma+|\alpha|)}\right]
$$


holds for $x \in B_{R_{0}}^{c}:=\mathbb{R}^{3} \backslash B_{R_{0}}, t \in\left(0, T_{0}\right), \quad \alpha \in \mathbb{N}_{0}^{3}$ with $|\alpha|:=\alpha_{1}+\alpha_{2}+\alpha_{3} \leq 1$, where the function $\nu(x):=1+|x|-x_{1}\left(x \in \mathbb{R}^{3}\right)$ should be considered as a mathematical manifestation of the wake extending downstream from the rigid body. The real number $R_{0}$ must be sufficiently large so that $\bar{\Omega} \subset B_{R_{0}}$. The condition $|\alpha| \leq 1$ means that inequality (1.3) covers the velocity $u$ itself as well as its spatial gradient $\nabla_{x} u$. The number $\gamma$ in the term $|x|^{-(\gamma+|\alpha|)}$ equals 3 if the zero flux condition

$$
\int_{\partial \Omega} u(t) \cdot n^{(\Omega)} d o_{x} \quad\left(t \in\left(0, T_{0}\right)\right)
$$

holds; otherwise $\gamma=2$. (By $n^{(\Omega)}$, we denote the outward unit normal to $\Omega$.) The parameters $\sigma_{1}, \sigma_{2}, \sigma_{3} \in[1, \infty], q \in(1, \infty)$ in (1.3) are introduced via the assumptions $u\left|Z_{R_{1}, T_{0}} \in L^{\sigma_{1}}\left(0, T_{0}, L^{q}\left(\Omega_{R_{1}}\right)^{3}\right), \quad \nabla_{x} u\right| Z_{R_{1}, T_{0}} \in L^{\sigma_{2}}\left(0, T_{0}, L^{q}\left(\Omega_{R_{1}}\right)^{3}\right)$ and $f \mid Z_{R_{1}, T_{0}} \in$ $L^{\sigma_{3}}\left(0, T_{0}, L^{q}\left(\Omega_{R_{1}}\right)^{3}\right)$, where $\Omega_{R_{1}}:=B_{R_{1}} \backslash \bar{\Omega}$ and $Z_{R_{1}, T_{0}}:=\Omega_{R_{1}} \times(0, \infty)$, for some $R_{1} \in$ $(0, \infty)$ with $\bar{\Omega} \subset B_{R_{1}}$. The appearance of these parameters $\sigma_{j}$ in (1.3) means that the spatial decay of $u$ and $\nabla_{x} u$ depends on $L^{p}$-integrability in time of $u\left|Z_{R_{1}, T_{0}}, \nabla_{x} u\right| Z_{R_{1}, T_{0}}$ and $f \mid Z_{R_{1}, T_{0}}$. This is the link between $L^{p}$-integrability in time and pointwise decay in space of $u$ alluded to in the title of this work. Another key point of our theory is that we do not impose any boundary conditions on $u$ or $\pi$, except that we consider the case that the zero flux condition (1.4) is valid. If that condition holds and if $|f(x, t)|$ and $|u(x, 0)|$ decrease sufficiently fast for $|x| \rightarrow \infty$, then inequality (1.3) implies that

$$
\left|\partial_{x}^{\alpha} u(x, t)\right| \leq \mathfrak{C}(|x| \nu(x))^{-(3+|\alpha|) / 2+1 /\left(2 \min \left\{\sigma_{1}^{\prime}, \sigma_{2}^{\prime}\right\}\right)}
$$

for $x, t, \alpha$ as before. For more details, we refer to Theorem 6.1.

We chose our assumptions exclusively in view of establishing (1.3), without any intent of proving existence results for solutions to (1.1). But of course, solutions to (1.1) fulfilling our requirements should be known to exist. We state some results in this respect in Theorem 6.2 and 6.3, with suitable references as proof.

In a successor paper [17], we will show that inequality (1.3) remains valid if $u$ is only (the velocity part of) a $L^{q}$-weak solution to (1.1). A further successor paper [19] extends (1.3) to mild solutions to (1.1), under homogeneous Dirichlet boundary conditions. In still another successor paper [18], we deal with the nonlinear stability problem

$$
\begin{aligned}
& \partial_{t} u-\Delta_{x} u+\tau \partial_{x_{1}} u+\tau\left(U \cdot \nabla_{x}\right) u+\tau(u \cdot \nabla) U+\tau\left(u \cdot \nabla_{x}\right) u+\nabla_{x} \pi=f, \\
& \operatorname{div}_{x} u=0 \text { in } \bar{\Omega}^{c} \times\left(0, T_{0}\right),
\end{aligned}
$$

where $U: \bar{\Omega}^{c} \mapsto \mathbb{R}^{3}$ is the velocity part of a solution to the stationary Navier-Stokes system

$$
-\Delta U+\tau \partial_{1} U+\tau(U \cdot \nabla) U+\nabla \Pi=F, \operatorname{div} U=0 \text { in } \bar{\Omega}^{c} .
$$

Note that (1.6) reduces to (1.2) if $U=0$. We show in [18] that $L^{2}$-strong solutions to (1.6) satisfy (1.3) with $p=2$. These successor papers [17], [19], [18] build on the results established here, so the work at hand is of interest not only in its own right, but also because it is the foundation of the theory derived in these latter articles.

Let us compare the results and the method of proof in the work at hand with related theories available in literature. What has been established up to now are estimates of the 
form

$$
\left|\partial_{x}^{\alpha} u(x, t)\right| \leq \mathfrak{C}(|x| \nu(x))^{-1-|\alpha| / 2} \quad \text { for } x \in B_{R_{0}}^{c}, \quad t \in\left(0, T_{0}\right),
$$

starting with Mizumachi [36, Theorem 2], who identified a class of initial data $U_{0}$ and a class of solutions $(u, \pi)$ to the nonlinear problem (1.2) with $f=0$ such that $u$ satisfies (1.8) in the case $\alpha=0$. In [10] and [13], we established (1.8) for $\alpha \in \mathbb{N}_{0}^{3}$ with $|\alpha| \leq 1$ if $u$ is an $L^{2}$-weak solution to the Oseen system (1.1) under Dirichlet boundary conditions with data verifying (1.4), and in [14], we derived (1.8) for the same range of $\alpha$ in the case that $u$ is an $L^{2}$-strong solution to the stability system (1.6), also under Dirichlet boundary conditions with data satisfying (1.4).

The proofs in all those references, and also in the work at hand, rely on integral representations of the velocity part $u$ of solutions to the time-dependent Oseen system (1.1). When equations (1.2) or (1.6) are studied, the additional terms in these systems are considered as part of the right-hand side of (1.1). However, these articles differ with respect to the choice of such a representation. Mizumachi [36] used a Green's formula, a variant of which appears in the present article as equation (5.4). Such an equation has the disadvantage that it involves an integral on $\partial \Omega \times\left(0, T_{0}\right)$ of $\nabla_{x} u$ and $\pi$. This is the reason why in [36], the restrictive integrability conditions mentioned above are imposed on $\nabla_{x} u \mid \partial \Omega \times\left(0, T_{0}\right)$ and $\pi \mid \partial \Omega \times\left(0, T_{0}\right)$. In [10], [13] and [14], we circumvented this difficulty by solving an integral equation in a certain subspace of $L^{2}\left(0, T_{0}, L^{2}(\partial \Omega)^{3}\right)$. This approach provides a representation formula for solutions to (1.1) which does not contain the critical integrals mentioned above. In addition it even yields existence of a solution to (1.1) under Dirichlet boundary conditions satisfying (1.4). However, it is limited to an $L^{2}$-framework, and even in that context, the decay rate $-1-|\alpha| / 2$ it provides as indicated in (1.8) is not optimal. Concerning this latter point, in the case of homogeneous Dirichlet boundary conditions, the theory in the present paper yields the stronger decay rate $-5 / 4-|\alpha| / 2$, albeit under assumptions on $U_{0}$ and $f$ somewhat different from those in [10] and [13]. We refer to Theorem 6.2 and the comment preceding it for more details. In [17], the theory presented in the work at hand is extended to a more general framework covering the situation in [10] and [13]. It turned out that we again obtain the higher rate $-5 / 4-|\alpha| / 2([17$, Theorem $6.1,6.2])$, compared with $-1-|\alpha| / 2$ in [10] and [13].

A similar comparison is valid between [14] and the successor paper [18] to the present work: As already indicated further above, it is shown in [14] that for $L^{2}$-strong solutions to (1.6) satisfying (1.4), the estimate in (1.8) holds, so the velocity decays with the rate $-1-|\alpha| / 2$. On the other hand, in [18] we will prove that (1.3) holds with $\sigma_{1}=\sigma_{2}=2$, hence the velocity decreases with the rate $-5 / 4-|\alpha| / 2$ if (1.4) is satisfied.

The improvements derived in the present work, in [17], [19] and [18] compared to existing theory in [36], [10], [13] and [14] - higher decay rates, no boundary conditions involved except (1.4) if so chosen, transition from an $L^{2}$ - to an $L^{q}$-framework in the linear case, decay estimates for mild solutions to (1.1) - are essentially due to a representation formula (equation (5.7)) which is different from the ones used in those earlier references. Since it is derived from a Green's formula, this equation does not require solving an integral equation. Still it does not contain the critical boundary integrals mentioned above because they are removed by partial integration and a cut-off procedure; see the proof of Corollary 5.1. Although this approach introduces a restriction, too - the velocity is represented at a 
point $(x, t)$ only if $x$ is located outside a fixed ball around $\bar{\Omega}-$, this does not matter in our context because we are interested in the behaviour of the velocity in $(x, t)$ for large values of $|x|$.

A similar representation formula was derived in [15] for solutions to the time-dependent Stokes system; see [15, Theorem 4.3]. However, this formula only leads to pointwise decay estimates of the velocity itself, but not of its spatial gradient, and is valid only if $f$ vanishes and homogeneous Dirichlet boundary conditions are satisfied. Moreover the theory in [15] is essentially restricted to an $L^{2}$-framework and is based on maximal regularity of solutions to the time-dependent Stokes system. Maximal regularity cannot be expected to hold for solutions to the Oseen system (1.1). In fact, according to [20], the velocity part $U$ of a solution $(U, \Pi)$ to the Oseen resolvent system $-\Delta U+\tau \partial_{1} U+\lambda U+\nabla \Pi=F, \operatorname{div} U=0$ in the whole space $\mathbb{R}^{3}$ does not satisfy the estimate $\|U\|_{2} \leq C_{0}|\lambda|^{-1}\|F\|_{2}$ with a single constant $C_{0}>0$ for all $F \in L^{2}\left(\mathbb{R}^{3}\right)^{3}$ and all $\lambda \in \mathbb{C}$ with $\Re \lambda>0$. As a consequence of this negative result, which arises since small values of $|\lambda|$ are admitted, an analogous resolvent estimate cannot be expected to hold for solutions to the Oseen resolvent problem in $\bar{\Omega}^{c}$, under whatever boundary conditions. Therefore, in view of [40, Theorem 4.2, point 3.)], it is a safe guess that maximal regularity is not valid for solutions of problem (1.1), not even in an $L^{2}$-framework.

Let us mention some references more distantly related to the work at hand. Knightly [31] considered even the case that the velocity of the rigid body changes with time. However, his results are valid only under various smallness assumptions. Takahashi [43] deals with (1.6) in the case $\Omega=\emptyset$ under a smallness condition. In [2], [3], solutions to (1.1) and (1.6) are estimated in weighted $L^{p}$-norms, with the weights adapted to the wake in the flow field downstream to the rigid body. Reference [16] by the present author combines decay estimates in time and in space, as a continuation of [13] (Oseen system (1.1)) and [14] (problem (1.6)), with the same assumptions and methods as in those latter articles. Various technical aspects of the theory in [10], [13], [14] and [16] are dealt with in predecessor papers [6] - [9], [11], [12]. Questions of existence, regularity and stability related to (1.1), (1.2) or (1.6) are addressed in [23], [24], [25], [28], [29], [32], [34], [35], [39], [42].

\section{Notation. Some auxiliary results.}

The symbol || denotes the Euclidean norm of $\mathbb{R}^{n}$ for any $n \in \mathbb{N}$, the length $\alpha_{1}+\alpha_{2}+\alpha_{3}$ of a multi-index $\alpha \in \mathbb{N}_{0}^{3}$, and the Borel measure of measurable subsets of $\mathbb{R}^{3}$. For $R \in$ $(0, \infty), x \in \mathbb{R}^{3}$, put $B_{R}(x):=\left\{y \in \mathbb{R}^{3}:|x-y|<R\right\}$. In the case $x=0$, we write $B_{R}$ instead of $B_{R}(0)$.

Recall that in Section 1, we introduced the function $\nu: \mathbb{R}^{3} \mapsto[1, \infty)$ by setting $\nu(x):=$ $1+|x|-x_{1}$ for $x \in \mathbb{R}^{3}$.

We fix numbers $S_{0}, R_{0} \in(0, \infty)$ with $S_{0}<R_{0}$, as well as a function $\varphi_{0} \in C_{0}^{\infty}\left(B_{\left(R_{0}+S_{0}\right) / 2}\right)$ with $0 \leq \varphi_{0} \leq 1$ and $\varphi_{0} \mid B_{S_{0}+\left(R_{0}-S_{0}\right) / 4}=1$. The real number $R_{1}:=\left(R_{0}+S_{0}\right) / 2$ will be used frequently.

The parameters $T_{0} \in(0, \infty]$ and $\tau \in(0, \infty)$ as well as the open bounded set $\Omega \subset \mathbb{R}^{3}$ with Lipschitz boundary, all of them introduced in Section 1, will be kept fixed throughout. 
Further recall that the outward unit normal to $\Omega$ is denoted by $n^{(\Omega)}$. Set $\Omega_{R}:=B_{R} \backslash \bar{\Omega}$ for $R \in\left[S_{0}, \infty\right)$. We assume that $\Omega \subset B_{S_{0}}$. In particular the case $\Omega=B_{S_{0}}$ is admitted.

If $R, S \in(0, \infty)$ with $S<R$, we write $A_{R, S}$ for the annular domain $B_{R} \backslash \overline{B_{S}}$. For $n \in$ $\mathbb{N}, I \subset \mathbb{R}^{n}$, let $\chi_{I}$ stand for the characteristic function of $I$ in $\mathbb{R}^{n}$. If $A \subset \mathbb{R}^{3}$, we denote by $A^{c}$ the complement $\mathbb{R}^{3} \backslash A$ of $A$ in $\mathbb{R}^{3}$. Put $e_{l}:=\left(\delta_{j l}\right)_{1 \leq j \leq 3}$ for $1 \leq l \leq 3$ (unit vector in $\mathbb{R}^{3}$ ). If $A$ is some nonempty set and $\gamma: A \mapsto \mathbb{R}$ a function, we set $|\gamma|_{\infty}:=\sup \{|\gamma(x)|: x \in A\}$.

Let $p \in[1, \infty)$ and $m \in \mathbb{N}$. For any open set $A \subset \mathbb{R}^{3}$, the norm of the Lebesgue space $L^{p}(A)$ is denoted by \|\|$_{p}$, and the expression \|\|$_{m, p}$ stands for the usual norm of the Sobolev space $W^{m, p}(A)$ of order $m$ and exponent $p$. The spaces $L_{l o c}^{p}(A)$ and $W_{l o c}^{m, p}(A)$, again for $A \subset \mathbb{R}^{3}$ open, are defined as the set of all functions $V$ from $A$ into $\mathbb{R}$ such that $V \mid K \in L^{p}(K)$ and $V \mid K \in W^{1, p}(K)$, respectively, for any open, bounded set $K \subset \mathbb{R}^{3}$ with $\bar{K} \subset A$. We put $\nabla V:=\left(\partial_{k} V_{j}\right)_{1 \leq j, k \leq 3}$ for $V \in W_{l o c}^{1,1}(A)^{3}$.

Let $\mathcal{V}$ be a normed space, and let the norm of $\mathcal{V}$ be denoted by \|\| . Then we will use the same notation \|\| for the norm on $\mathcal{V}^{n}$ defined by $\left\|\left(f_{1}, \ldots, f_{n}\right)\right\|:=\left(\sum_{j=1}^{n}\left\|f_{j}\right\|^{2}\right)^{1 / 2}$ for $\left(f_{1}, \ldots, f_{n}\right) \in \mathcal{V}^{n}(n \in \mathbb{N})$. The space $\mathcal{V}^{3 \times 3}$, as concerns its norm, is identified with $\mathcal{V}^{9}$.

Again for open sets $A \subset \mathbb{R}^{3}$, we define $C_{0, \sigma}^{\infty}(A):=\left\{V \in C_{0}^{\infty}(A)^{3}: \operatorname{div} V=0\right\}$, and we write $L_{\sigma}^{p}(A)$ for the closure of $C_{0, \sigma}^{\infty}(A)$ with respect to the norm of $L^{p}(A)^{3}$, where $p \in(1, \infty)$. This function space $L_{\sigma}^{p}(A)$ ("space of solenoidal $L^{p}$-functions") is equipped with the norm \|\|$_{p}$.

Let $p \in[1, \infty]$ and $\mathcal{B}$ a Banach space. For any interval $J \subset \mathbb{R}$, the norm of $L^{p}(J, \mathcal{B})$ is denoted by \|\|$_{L^{p}(J, \mathcal{B})}$. Let $a, b \in \mathbb{R} \cup\{\infty\}$ with $a<b$, and let $q \in[1, \infty)$. We write $L^{p}(a, b, \mathcal{B})$ and $W^{1, q}(a, b, \mathcal{B})$ instead of $L^{p}((a, b), \mathcal{B})$ and $W^{1, q}((a, b), \mathcal{B})$, respectively. The space $L_{l o c}^{p}([a, b), \mathcal{B})$ is to contain all functions $v:(a, b) \mapsto \mathcal{B}$ such that $v \mid(a, T) \in L^{p}(a, T, \mathcal{B})$ for any $T \in(a, b)$. Obviously this space is different from the standard space $L_{l o c}^{p}(a, b, \mathcal{B})$, which will also appear. The space $W_{l o c}^{1, q}([a, b), \mathcal{B})$ is to be defined analogously as $L_{\text {loc }}^{p}([a, b), \mathcal{B})$ and is to be distinguished from the space $W_{\text {loc }}^{1, q}(a, b, \mathcal{B})$. If $v \in W^{1,1}(a, b, \mathcal{B})$, then, possibly after a modification on a subset of $(a, b)$ with measure zero, the function $v$ belongs to $C^{0}([a, b), \mathcal{B})([44$, Lemma 3.1.1]). If the latter relation is already valid, we write $v \in W^{1,1}(a, b, \mathcal{B}) \cap C^{0}([a, b), \mathcal{B})$.

Let $T \in(0, \infty], A \subset \mathbb{R}^{3}$ open, $p, q \in[1, \infty], n \in \mathbb{N}$. Then we will write \|\|$_{q, p ; T}$ instead of \|\|$_{L^{p}\left(0, T, L^{q}(A)^{n}\right)}$. Of course, for an interval $J \subset \mathbb{R}$, a function $v \in L^{p}\left(J, L^{q}(A)^{n}\right)$ may be considered also as a function on $A \times J$, although there is a minor issue with respect to measurability on $A \times J$, settled in [11, Lemma 2.1] and Lemma 2.3. For $x \in A, t \in J$, we will write $v(t)(x)$ or $v(x, t)$ depending on whether $v$ is considered as a function on $J$ with values in $L^{q}(A)^{n}$, or as a function on $A \times J$. If $v \in W_{l o c}^{1,1}\left(J, L^{q}(A)^{n}\right)$, we write $v^{\prime}$ for the weak derivative of $v: J \mapsto L^{q}(A)^{n}$, and $\partial_{t} v$ for the weak partial derivative of $v$ as a funtion on $A \times J$; compare [15, Lemma 2.3]. For a function $v: J \mapsto W_{l o c}^{1,1}(A)^{3}$, the notation $\nabla_{x} v$ stands for the gradient of $v$ with respect to $x \in A$, in the sense that

$$
\nabla_{x} v: J \mapsto L_{l o c}^{1}(A)^{3 \times 3}, \nabla_{x} v(t)(x):=\left(\partial x_{k}\left(v_{j}(t)\right)(x)\right)_{1 \leq j, k \leq 3} \text { for } t \in J, x \in A
$$

(spatial gradient of $v$ ). Similar conventions are to be valid with respect to the expressions $\Delta_{x} v, \operatorname{div}_{x} v$ and $\partial x_{j} v$. 
Concerning Bochner integrals, if $J \subset \mathbb{R}$ is open, $\mathcal{B}$ a Banach space and $w: J \mapsto \mathcal{B}$ an integrable function, it will sometimes be convenient to write $\mathcal{B}-\int_{J} w(t) d t$ instead of $\int_{J} w(t) d t$ for the corresponding $\mathcal{B}$-valued Bochner integral. For the definition of the Bochner integral, we refer to [46, p. 132-133], or to [30, p. 78-80.].

We write $C$ for numerical constants and $C\left(\gamma_{1}, \ldots, \gamma_{n}\right)$ for constants depending exclusively on paremeters $\gamma_{1}, \ldots, \gamma_{n} \in[0, \infty)$ for some $n \in \mathbb{N}$. However, such a precise bookkeeping will be possible only at some places. Mostly we will use the symbol $\mathfrak{C}$ for constants whose dependence on parameters must be traced from context. Sometimes we write $\mathfrak{C}\left(\gamma_{1}, \ldots, \gamma_{n}\right)$ in order to indicate that the constants in question is influenced by the quantities $\gamma_{1}, \ldots, \gamma_{n}$. But in such cases, this constant depends on other parameters as well.

The following simple version of Young's inequality for integrals will be used frequently. Stated her for the convenience of the reader, we will refer to it as "Young's inequality".

Lemma 2.1 ([1, Corollary 2.25]) Let $n \in \mathbb{N}$ and $q \in[1, \infty]$. Then

$$
\left(\int_{\mathbb{R}^{n}}\left|\int_{\mathbb{R}^{n}} U(x-y) V(y) d y\right|^{q} d x\right)^{1 / q} \leq C\|U\|_{1}\|V\|_{q} \quad \text { for } U \in L^{1}\left(\mathbb{R}^{n}\right), V \in L^{q}\left(\mathbb{R}^{n}\right) .
$$

Another tool which will be used often is Minkowski's inequality for integrals. Again for the convenience of the reader, we recall it here.

Theorem 2.1 ([1, Theorem 2.9]) Let $m, n \in \mathbb{N}, p \in[1, \infty), F: \mathbb{R}^{n} \times \mathbb{R}^{m} \mapsto \mathbb{R} a$ measurable function. Then

$$
\left(\int_{\mathbb{R}^{n}}\left(\int_{\mathbb{R}^{m}}|F(x, y)| d y\right)^{p} d x\right)^{1 / p} \leq \int_{\mathbb{R}^{m}}\left(\int_{\mathbb{R}^{n}}|F(x, y)|^{p} d x\right)^{1 / p} d y .
$$

For vector-valued functions integrable on exterior domains with integrable divergence, the divergence theorem holds:

Lemma 2.2 Let $A \subset \mathbb{R}^{3}$ be open and bounded, with Lipschitz boundary. Denote the outward unit normal to $A$ by $n^{(A)}$. Let $V \in W_{\text {loc }}^{1,1}\left(\bar{A}^{c}\right)^{3} \cap L^{1}\left(\bar{A}^{c}\right)^{3}$ with $\operatorname{div} V \in L^{1}\left(\bar{A}^{c}\right)$. Further suppose that $\nabla V \mid B_{R} \backslash \bar{A}$ belongs to $L^{1}\left(B_{R} \backslash \bar{A}\right)^{9}$ for $R \in(0, \infty)$ with $\bar{A} \subset B_{R}$. Then $\int_{\bar{A}^{c}} \operatorname{div} V d x=-\int_{\partial A} V \cdot n_{k}^{(A)} d o_{x}$.

Proof: Fix some $\psi \in C_{0}^{\infty}\left(B_{2}\right)$ with $\psi \mid B_{1}=1$. Put $\psi_{n}(x):=\psi\left(n^{-1} x\right)$ for $x \in \mathbb{R}^{3}, n \in \mathbb{N}$. Then $\int_{\bar{A}^{c}} \operatorname{div} V d x=\lim _{n \rightarrow \infty} \int_{\bar{A}^{c}} \operatorname{div} V \psi_{n} d x$ by Lebesgue's theorem, and

$$
\int_{\bar{A}^{c}} \operatorname{div} V \psi_{n} d x=-\int_{\partial A} V \cdot n^{(A)} d o_{x}-\int_{\bar{A}^{c}} V \cdot \nabla \psi_{n} d x \quad \text { for } n \in \mathbb{N} \text { with } \bar{A} \subset B_{n} .
$$

But $\left|\partial_{k} \psi_{n}(x)\right| \leq\left|\partial_{k} \psi\right|_{\infty} n^{-1} \chi_{B_{2 n} \backslash B_{n}}(x)$ for $n \in \mathbb{N}, x \in \mathbb{R}^{3}, 1 \leq k \leq 3$, so $\int_{\bar{A}^{c}} V \cdot \nabla \psi_{n} d x \rightarrow$ $0(n \rightarrow \infty)$ again by Lebesgue's theorem.

We note that functions $V$ from $W_{l o c}^{1,1}\left(\mathbb{R}^{3}\right)$ with $\nabla V \in L^{q}\left(\mathbb{R}^{3}\right)^{3}$ may be approximated in the $L^{q}$-norm by the gradient of $C_{0}^{\infty}$-functions in $\mathbb{R}^{3}$.

Theorem 2.2 ([38, Lemma I.1.1]) Let $q \in(1, \infty)$ and $V \in W_{l o c}^{1,1}\left(\mathbb{R}^{3}\right)$ with $\nabla V \in$ $L^{q}\left(\mathbb{R}^{3}\right)^{3}$. Then there is a sequence $\left(V_{n}\right)$ in $C_{0}^{\infty}\left(\mathbb{R}^{3}\right)$ with $\left\|\nabla\left(V_{n}-V\right)\right\|_{q} \rightarrow 0$.

We will make use of the Helmholtz-Fujita decomposition of $L^{q}$-functions in the whole space $\mathbb{R}^{3}$. 
Theorem 2.3 Let $q \in(1, \infty)$. Then there are linear operators $P_{q}: L^{q}\left(\mathbb{R}^{3}\right)^{3} \mapsto L_{\sigma}^{q}\left(\mathbb{R}^{3}\right)$ and $G_{q}: L^{q}\left(\mathbb{R}^{3}\right)^{3} \mapsto W_{\text {loc }}^{1, q}\left(\mathbb{R}^{3}\right)$ such that $\nabla G_{q}(V) \in L^{q}\left(\mathbb{R}^{3}\right)^{3}, V=P_{q}(V)+\nabla G_{q}(V)$ and $\left\|P_{q}(v)\right\|_{q}+\left\|\nabla G_{q}(V)\right\|_{q} \leq C(q)\|V\|_{q}$ for $V \in L^{q}\left(\mathbb{R}^{3}\right)^{3}$.

Proof: See [27, Section III.1], in particular [27, p. 147-148 and Theorem III.1.2].

In the next theorem, the equation $\operatorname{div} V=F$ is solved in $W_{0}^{1, q}(A)^{3}$, where $A$ is an annular domain.

Theorem 2.4 Let $S, R \in(0, \infty)$ with $S<R$, and put $A:=A_{R, S}$. For $q \in(1, \infty)$, define the space $L_{0}^{q}(A)$ by setting $L_{0}^{q}(A):=\left\{F \in L^{q}(A): \int_{A} F d x=0\right\}$. Then there is an operator $\mathfrak{D}=\mathfrak{D}_{R, S}$ from $\cup\left\{L_{0}^{q}(A): q \in(1, \infty)\right\}$ into $\cup\left\{W_{0}^{1, q}(A)^{3}: q \in(1, \infty)\right\}$ with the following properties.

The restriction $\mathfrak{D} \mid L_{0}^{q}(A)$ maps into $W_{0}^{1, q}(A)^{3}$, for any $q \in(1, \infty)$,

$\operatorname{div} \mathfrak{D}(F)=F$ for $F \in L_{0}^{q}(A), q \in(1, \infty)$, and

$\mathfrak{D}(F) \in C_{0}^{\infty}(A)^{3}$ for $F \in C_{0}^{\infty}(A)$ with $\int_{A} F d x=0$.

Proof: Abbreviate $C_{0,0}^{\infty}:=\left\{F \in C_{0}^{\infty}(A): \int_{A} F d x=0\right\}$. According to [4, Theorem 2.4], for any $q \in(1, \infty)$, there is a linear and bounded operator $\mathfrak{D}_{q}: L_{0}^{q}(A) \mapsto W_{0}^{1, q}(A)^{3}$ such that $\operatorname{div} \mathfrak{D}_{q}(F)=F$ for $F \in L_{0}^{q}(A)$, and such that for any $F \in C_{0,0}^{\infty}$, the function $D_{q}(F)$ belongs to $C_{0}^{\infty}(A)^{3}$ and only depends on $R, S$ and $F$. Let $p, q \in(1, \infty)$. By the preceding statement, the operators $\mathfrak{D}_{p}$ and $\mathfrak{D}_{q}$ coincide on $C_{0,0}^{\infty}$. Suppose that $q \geq p$, and let $F \in L_{0}^{q}(A) \cap L_{0}^{p}(A)$. There is a sequence $\left(F_{n}\right)$ in $C_{0}^{\infty}(A)$ such that $\left\|F_{n}-F\right\|_{q} \rightarrow 0$. Since $A$ is bounded, this means in particular that $\int_{A} F_{n} d x \rightarrow \int_{A} F d x=0$. Fix a function $\varphi \in C_{0}^{\infty}(A)$ with $\int_{A} \varphi d x=1$, and put $\widetilde{F}_{n}:=F_{n}-\left(\int_{A} F_{n} d x\right)\left(\int_{A} \varphi d x\right)^{-1} \varphi$ for $n \in \mathbb{N}$. Then $\widetilde{F}_{n} \in C_{0,0}^{\infty}$ for $n \in \mathbb{N}$ and $\left\|\widetilde{F_{n}}-F\right\|_{q} \rightarrow 0$. But $\mathfrak{D}_{p}$ and $\mathfrak{D}_{q}$ coincide on $C_{0,0}^{\infty}$, as mentioned above, and these two operators are bounded. In addition, $A$ is bounded and $p \leq q$. So we obtain $\mathfrak{D}_{q}(F)=\mathfrak{D}_{p}(F)$. This means that the operators $\mathfrak{D}_{p}$ and $\mathfrak{D}_{q}$ coincide on $L_{0}^{q}(A) \cap L_{0}^{p}(A)$ as well. Hence there exists an operator with properties as stated in the theorem.

Let us recall some properties of the Bochner integral that will be important in what follows. First we recall that Bochner integration commutes with bounded operators.

Theorem 2.5 Let $B_{1}, B_{2}$ be Banach spaces, $A: B_{1} \mapsto B_{2}$ a linear and bounded operator, $n \in \mathbb{N}, J \subset \mathbb{R}^{n}$ an open set and $f: J \mapsto B_{1}$ a Bochner integrable mapping. Then $A \circ f: J \mapsto B_{2}$ is Bochner integrable, too, and $A\left(B_{1}-\int_{J} f d x\right)=B_{2}-\int_{J} A \circ f d x$.

Proof: See [46, p. 134, Corollary 2], [30, Theorem 3.7.12].

We will sometimes interprete $L^{q}(U)$-valued Bochner integrals as standard Lebesgue integrals. In view of its importance in the present context, and for completeness, we briefly discuss this transition. In particular, we indicate why a measurable function $f: J \mapsto L^{q}(U)$ is measurable as a function on $J \times U$, where $J \subset \mathbb{R}^{n}$ is open, for some $n \in \mathbb{N}$. This feature is often used in the work at hand with respect to functions from $L_{l o c}^{1}\left(J, L^{q}(U)\right)$ when $J$ is an interval in $\mathbb{R}$. In [15, Lemma 3.5], we already considered the case $q=2$, without treating measurability. In the proof of the ensuing lemma, we simplify the argument from [15] by applying Theorem 2.5. 
Lemma 2.3 Let $m, n \in \mathbb{N}, J \subset \mathbb{R}^{n}$ and $U \subset \mathbb{R}^{m}$ open sets, $q \in[1, \infty)$ and $f: J \mapsto$ $L^{q}(U)^{3}$ integrable as a Bochner integral in $L^{q}(U)^{3}$. Then there is a measurable function $g: J \times U \mapsto \mathbb{R}^{3}$ such that $f(t)=g(t)$ a. e. in $U$, for a. e. $t \in J$. We identify $f$ with $g$. Then $\int_{J}|f(t)(x)| d t<\infty$ and $\int_{J} f(t)(x) d t=\left(L^{q}(U)^{3}-\int_{J} f(t) d t\right)(x)$ for a. $e . x \in U$.

Proof: There is a sequence $\left(f_{k}\right)$ of simple functions from $J$ into $L^{q}(U)^{3}$ such that the relation $\left\|\left(f_{k}-f\right)(t)\right\|_{q} \rightarrow 0(k \rightarrow \infty)$ holds for a. e. $t \in J$, and $\int_{J}\left\|\left(f_{k}-f\right)(t)\right\|_{q} d t \rightarrow$ $0(k \rightarrow \infty)$. (We recall that a function $g: J \mapsto L^{q}(U)^{3}$ is called simple if there is $k_{0} \in \mathbb{N}$, measurable subsets $E_{1}, \ldots, E_{k_{0}}$ of $J$ and functions $V_{1}, \ldots, V_{k_{0}} \in L^{q}(U)^{3}$ such that $\left|E_{j}\right|<\infty$ for $1 \leq j \leq k_{0}$ and $g(t)=\sum_{j=1}^{k_{0}} \chi_{E_{j}}(t) V_{j}$ for $t \in J$.)

Let $N \in \mathbb{N}$ with $U \cap B_{N} \neq \emptyset$, and put $U_{N}:=U \cap B_{N}$. Then the function $f_{k} \mid J \times U_{N}$ is measurable and belongs to $L^{1}\left(J \times U_{N}\right)^{3}$, for $k \in \mathbb{N}$. Moreover $\left\|\left(f_{k}-f\right)(t) \mid U_{N}\right\|_{1} \rightarrow 0(k \rightarrow$ $\infty)$ for a. e. $t \in J$, and $\int_{J} \int_{U_{N}}\left|\left(f_{k}-f_{l}\right)(t)(x)\right| d x d t \rightarrow 0(k, l \rightarrow \infty)$. The latter relation implies there is $g_{N} \in L^{1}\left(J \times U_{N}\right)^{3}$ with $\left\|g_{N}-f_{k}\right\|_{1} \rightarrow 0(k \rightarrow \infty)$. In particular there is a subsequence $\left(\widetilde{f}_{k}\right)$ of $\left(f_{k}\right)$ such that $\left\|\left(g_{N}-\widetilde{f}_{k}\right)(t) \mid U_{N}\right\|_{1} \rightarrow 0(k \rightarrow \infty)$ for a. e. $t \in J$. At this point we may conclude that $g_{N}(t)=f(t) \mid U_{N}$ a. e. on $U_{N}$ for a. e. $t \in J$. Since this is true for any $N \in \mathbb{N}$ with $U \cap B_{N} \neq \emptyset$, there is a measurable function $g: J \times U \mapsto \mathbb{R}^{3}$ such that $g(t)=f(t)$ a. e. on $U$ for a. e. $t \in J$.

Since $f: J \mapsto L^{p}(U)^{3}$ is integrable, the function $\|f(t)\|_{q}(t \in J)$ is integrable as well, so by Theorem 2.1, $\left(\int_{U}\left(\int_{J}|f(t)(x)| d t\right)^{p} d x\right)^{1 / p} \leq \int_{J}\|f(t)\|_{q} d t<\infty$. Hence $\int_{J}|f(t)(x)| d t<$ $\infty$ for a. e. $x \in U$, and the function $\int_{J} f(t)(x) d t(x \in U)$ belongs to $L_{l o c}^{1}(U)$. Let $\psi \in C_{0}^{\infty}(U)^{3}$. The mapping $\int_{U} \psi \cdot F d x\left(F \in L^{q}(U)\right)$ is linear and bounded, so by Theorem 2.5 and Fubini's theorem,

$$
\int_{U} \psi(x) \cdot\left(\int_{J} f(t) d t\right)(x) d x=\int_{J} \int_{U} \psi(x) \cdot f(t)(x) d x d t=\int_{U} \psi(x) \cdot \int_{J} f(t)(x) d t d x .
$$

This implies the equation stated in the lemma.

We will need mean continuity of the Bochner integral (proof of Theorem 2.7).

Theorem 2.6 ([30, Theorem 3.8.3]) Let $B$ be a Banach space and $f: \mathbb{R} \mapsto B$ a Bochner integrable function. Then $\int_{\mathbb{R}}\|f(s+h)-f(s)\|_{B} d s \rightarrow 0$ for $h \rightarrow 0$, where \|\|$_{B}$ denotes the norm of $B$.

We will use Friedrich's mollifier for functions with values in Banach spaces. Here are the relevant definitions: Fix a function $\varrho \in C_{0}^{\infty}((-1,1))$ with $\varrho \geq 0$ and $\int_{\mathbb{R}} \varrho(s) d s=1$, and put $\varrho_{\delta}(r):=\delta^{-1} \varrho\left(\delta^{-1} r\right)$ for $\delta \in(0, \infty), r \in \mathbb{R}$. If B is a Banach space and $f \in L_{\text {loc }}^{1}(\mathbb{R}, B)$, define $f_{\delta}(t):=B-\int_{\mathbb{R}} \varrho_{\delta}(t-s) f(s) d s$ for $t \in \mathbb{R}, \delta \in(0, \infty)$.

Key properties of Friedrich's mollifier of functions with values in $\mathbb{R}$ carry over to functions with values in Banach spaces. Such properties as needed in the work at hand are collected in the ensuing Theorem 2.7 and Lemma 2.4.

Theorem 2.7 Let $B$ be a Banach space and $f \in L_{\text {loc }}^{1}(\mathbb{R}, B)$. Then $f_{\delta} \in C^{\infty}(\mathbb{R}, B)$ and $f_{\delta}^{(n)}(t)=\int_{\mathbb{R}} \varrho_{\delta}^{(n)}(t-s) f(s) d s(n \in \mathbb{N}, t \in \mathbb{R}, \delta \in(0, \infty))$. If $f \in L^{1}(\mathbb{R}, B)$, then $\left\|f_{\delta}-f\right\|_{L^{1}(\mathbb{R}, B)} \rightarrow 0(\delta \downarrow 0)$. Moreover, if $f \in W_{\text {loc }}^{1,1}(\mathbb{R}, B)$, then $\left(f_{\delta}\right)^{\prime}=\left(f^{\prime}\right)_{\delta}$.

Proof: The relation $f_{\delta} \in C^{\infty}(\mathbb{R}, B)$ and the equation for $f_{\delta}^{(n)}(t)$ follow from Lebesgue's 
theorem and the fact that $\varrho_{\delta} \in C_{0}^{\infty}((-\delta, \delta))$. If $f \in L^{1}(\mathbb{R}, B)$, we may proceed in exactly the same way as in the case of functions with values in $\mathbb{R}$ (see [26, Section 2.5.3], for example) in order to deduce from Theorem 2.6 that $\left\|f_{\delta}-f\right\|_{L^{1}(\mathbb{R}, B)} \rightarrow 0(\delta \downarrow 0)$. If $f \in W_{\text {loc }}^{1,1}(\mathbb{R}, B)$ and $t \in \mathbb{R}$, the equation $\left(f_{\delta}\right)^{\prime}(t)=\left(f^{\prime}\right)_{\delta}(t)$ holds due to the above equation for $f_{\delta}^{(n)}(t)$ with $n=1$, and since the function $s \mapsto \varrho_{\delta}(t-s)(s \in \mathbb{R})$ belongs to $C_{0}^{\infty}(\mathbb{R})$.

Lemma 2.4 Let $B$ be a Banach space, $J \subset \mathbb{R}$ be an open interval, $f \in C^{0}(J, B), a, b \in J$ with $a<b$. Then $f_{\delta}(t) \rightarrow f(t)(\delta \downarrow 0)$ uniformly in $t \in[a, b]$.

Proof: The argument is the same as in the case $B=\mathbb{R}$; see [1, Section 2.29, p. 37-38] for example.

Friedrich's mollifier was introduced in order to establish the next lemma. Although it should be well known, we do not know a reference for it. Since on the other hand, this lemma will be used three times, in the proofs of Theorem 3.3, Lemma 5.1 and Corollary 5.1, we present a proof.

Lemma 2.5 Let $B$ be a Banach space, $a, b \in \mathbb{R}$ with $a<b, f \in W^{1,1}(a, b, B) \cap$ $C^{0}([a, b], B)$ and $G \in W^{1,1}\left(a, b, B^{\prime}\right) \cap C^{0}\left([a, b], B^{\prime}\right)$. Then

$$
\int_{a}^{b}\left[G^{\prime}(t)(f(t))+G(t)\left(f^{\prime}(t)\right)\right] d t=G(b)(f(b))-G(a)(f(a)) .
$$

Proof: Put $\tilde{f}(t):=f(a),\left(f^{\prime}\right)^{\sim}(t):=0$ for $t \in(-\infty, a), \tilde{f}(t):=f(t),\left(f^{\prime}\right)^{\sim}(t):=$ $f^{\prime}(t)$ for $t \in[a, b], \tilde{f}(t):=f(b),\left(f^{\prime}\right)^{\sim}(t):=0$ for $t \in(b, \infty)$. Let $\varphi \in C_{0}^{\infty}(\mathbb{R})$. Since $f \in W^{1,1}(a, b, B)$, a standard argument yields $\varphi f \in W^{1,1}(a, b, B)$ and $(\varphi f)^{\prime}=\varphi^{\prime} f+$ $\varphi f^{\prime}$. Obviously $\varphi f \in C^{0}([a, b], B)$. Let $T \in B^{\prime}$. With Theorem 2.5, we conclude that $T \circ(\varphi f) \in W^{1,1}((a, b)) \cap C^{0}([a, b])$ and $(T \circ(\varphi f))^{\prime}=T \circ\left(\varphi^{\prime} f+\varphi f^{\prime}\right)$. Hence we obtain $T\left(\int_{a}^{b}\left(\varphi^{\prime} f+\varphi f^{\prime}\right)\right)=T((\varphi f)(b)-(\varphi f)(a))$ by a standard result of analysis and Theorem 2.5. Since this is true for any $T \in B^{\prime}$, the preceding equation remains valid if $T$ is removed. Due to the definition of $\tilde{f}$ and $\left(f^{\prime}\right)^{\sim}$, it is now easy to conclude that $\int_{\mathbb{R}} \varphi^{\prime} f d s=$ $-\int_{\mathbb{R}} \varphi\left(f^{\prime}\right)^{\sim} d s$. Thus the weak derivative $\tilde{f}^{\prime}$ of $\tilde{f}$ exists and equals $\left(f^{\prime}\right)^{\sim}$, so that $\tilde{f} \in$ $W_{l o c}^{1,1}(\mathbb{R}, B)$ and $\tilde{f}^{\prime} \in L^{1}(\mathbb{R}, B)$. Theorem 2.7 now yields that $\left\|\tilde{f}^{\prime}-\left(\tilde{f}^{\prime}\right)_{\delta}\right\|_{L^{1}(\mathbb{R}, B)} \rightarrow 0(\delta \downarrow 0)$ and $\left(\tilde{f}^{\prime}\right)_{\delta}=\left(\tilde{f}_{\delta}\right)^{\prime}$. Since $\left(f^{\prime}\right)^{\sim} \mid(a, b)=f^{\prime}$, it follows that $\left\|f^{\prime}-\left(\tilde{f}_{\delta}\right)^{\prime} \mid(a, b)\right\|_{L^{1}(a, b, B)} \rightarrow 0(\delta \downarrow$ $0)$. Moreover $\tilde{f} \in C^{0}(\mathbb{R}, B)$ and $\tilde{f} \mid[a, b]=f$, so Lemma 2.4 implies that $\left\|f(t)-\tilde{f}_{\delta}(t)\right\|_{B} \rightarrow$ $0(\delta \downarrow 0)$ uniformly in $t \in[a, b]$, where \|\|$_{B}$ stands for the norm of $\mathrm{B}$.

Defining $\tilde{G}$ analogously as $\tilde{f}$, we may show in the same way that $\tilde{G} \in C^{0}\left(\mathbb{R}, B^{\prime}\right) \cap$ $W_{l o c}^{1,1}\left(\mathbb{R}, B^{\prime}\right), \tilde{G}^{\prime} \in L^{1}\left(\mathbb{R}, B^{\prime}\right), \tilde{G}_{\delta}(t) \rightarrow G(t)$ in $B^{\prime}$ uniformly with respect to $t \in[a, b]$, and $\left\|G^{\prime}-\left(\tilde{G}_{\delta}\right)^{\prime} \mid(a, b)\right\|_{L^{1}\left(a, b B^{\prime}\right)} \rightarrow 0$ for $\delta \downarrow 0$.

But for $\delta \in(0, \infty)$, the function $\psi_{\delta}(t):=\tilde{G}_{\delta}(t)\left(\tilde{f}_{\delta}(t)\right)(t \in \mathbb{R})$ belongs to $C^{1}(\mathbb{R})$, with $\psi_{\delta}^{\prime}(t)=\tilde{G}_{\delta}^{\prime}(t)\left(\tilde{f}_{\delta}(t)\right)+\tilde{G}_{\delta}(t)\left(\tilde{f}_{\delta}^{\prime}(t)\right)$, so equation (2.1) holds with $f, G$ replaced by $\tilde{f}_{\delta}, \tilde{G}_{\delta}$, respectively. In view of what has been shown above, this equation without these replacements follows when we let $\delta$ tend to 0 . 


\section{Fundamental solution to the time-dependent Oseen sys- tem. Related potential functions.}

In this section, we define a fundamental solution to the time-dependent Oseen system (1.1), and prove some estimates of this solution. Then we will introduce volume and layer potentials which will be constitutive elements of our representation formulas. We will prove properties of these potentials whose usefulness will become evident later on. To begin with, we indicate some properties of the function $\nu$ defined in Section 1, following (1.3).

Lemma 3.1 ([20, Lemma 4.8]) The inequality $\nu(x) \leq C(1+|y|) \nu(x-y)$ holds for $x, y \in \mathbb{R}^{3}$.

Lemma 3.2 ([6, Lemma 2]) Let $K \in(0, \infty)$. Then, for $r \in(0, \infty), z \in \mathbb{R}^{3}$,

$$
\begin{aligned}
& \left|z-\tau r e_{1}\right|^{2}+r \geq C(\tau, K)\left(|z|^{2}+r\right) \text { if }|z| \leq K, \\
& \left|z-\tau r e_{1}\right|^{2}+r \geq C(\tau, K)(|z| \nu(z)+r) \quad \text { if }|z| \geq K .
\end{aligned}
$$

Corollary 3.1 Let $R, R_{1} \in(0, \infty)$ with $R<R_{1}$, and take $x \in B_{R_{1}}^{c}, y \in B_{R}, r \in(0, \infty)$. Then $\left|x-y-\tau r e_{1}\right|^{2}+r \geq C\left(\tau, R, R_{1}\right)(|x| \nu(x)+r)$.

Proof: We have $|x-y| \geq|x|-|y| \geq\left(1-R / R_{1}\right)|x| \geq R_{1}-R>0$, so the corollary follows from Lemma 3.2 with $K=R_{1}-R$ and from Lemma 3.1.

Corollary 3.2 Let $\gamma \in(2, \infty), R \in(0, \infty)$ Then $\int_{B_{R}^{c}}(|x| \nu(x))^{-\gamma} d x \leq C(\gamma) R^{-\gamma+2}$.

Proof: By [25, Lemma 2.3], the estimate $\int_{\partial B_{r}} \nu(x)^{-\gamma} d o_{x} \leq C(\gamma) r$ holds for $r \in(0, \infty)$. Since $\int_{B_{R}^{c}}(|x| \nu(x))^{-\gamma} d x=\int_{R}^{\infty} r^{-\gamma} \int_{\partial B_{r}} \nu(x)^{-\gamma} d o_{x} d r$ and $\gamma>2$, the corollary follows.

As definition of the Newton kernel (fundamental solution of the Poisson equation), denoted by $\mathfrak{N}$ in this work, we choose this variant: $\mathfrak{N}(z):=(4 \pi|z|)^{-1}$ for $z \in \mathbb{R}^{3} \backslash\{0\}$. We introduce the usual heat kernel in $3 \mathrm{D}$,

$\mathfrak{H}(z, t):=(4 \pi t)^{-3 / 2} e^{-|z|^{2} /(4 t)}$ for $z \in \mathbb{R}^{3}, t \in(0, \infty), \quad \mathfrak{H}(z, 0):=0$ for $z \in \mathbb{R}^{3} \backslash\{0\}$.

Thus, in our context, $\mathfrak{H}$ is defined on $\mathfrak{B}:=\left(\mathbb{R}^{3} \times(0, \infty)\right) \cup\left(\left(\mathbb{R}^{3} \backslash\{0\}\right) \times\{0\}\right)$.

Theorem 3.1 The relations $\mathfrak{H} \in C^{\infty}(\mathfrak{B}), \int_{\mathbb{R}^{3}} \mathfrak{H}(z, t) d z=1$ for $t \in(0, \infty)$ hold. If $\alpha \in \mathbb{N}_{0}^{3}, \sigma \in \mathbb{N}_{0}$, the inequality $\left|\partial_{z}^{\alpha} \partial_{t}^{\sigma} \mathfrak{H}(z, t)\right| \leq C(\alpha, \sigma)\left(|z|^{2}+t\right)^{-(3+|\alpha|+2 \sigma) / 2}$ is valid for $z \in \mathbb{R}^{3}, t \in(0, \infty)$.

Proof: See [41] for the preceding estimate.

We will use the estimate in Theorem 3.1 only in the case $|\alpha| \leq 3, \sigma \in\{0,1,2\}$. In view of Theorem 3.1, we may define the following velocity part $\Gamma$ of a fundamental solution to the time-dependent Stokes system:

$$
\Gamma_{j k}(z, t):=\mathfrak{H}(z, t) \delta_{j k}+\int_{t}^{\infty} \partial z_{j} \partial z_{k} \mathfrak{H}(z, s) d s \quad \text { for } \quad(z, t) \in \mathfrak{B}, j, k \in\{1,2,3\} .
$$


Theorem 3.2 The relation $\Gamma \in C^{\infty}(\mathfrak{B})^{3 \times 3}$ holds. If $\alpha \in \mathbb{N}_{0}^{3}, \sigma \in \mathbb{N}_{0}$, the estimate $\left|\partial_{z}^{\alpha} \partial_{t}^{\sigma} \Gamma(z, t)\right| \leq C(\alpha, \sigma)\left(|z|^{2}+t\right)^{-(3+|\alpha|+2 \sigma) / 2}$ is fulfilled for $z \in \mathbb{R}^{3}, t \in(0, \infty)$. Moreover $\sum_{k=1}^{3} \partial z_{k} \Gamma_{j k}(z, t)=0, \partial_{t} \Gamma(z, t)-\Delta_{z} \Gamma(z, t)=0$ for $z, t$ as before, and for $1 \leq j \leq 3$.

Proof: Use Theorem 3.1, or see [37, Proposition 2.1.9].

As in the case of the inequality for $\mathfrak{H}$ in Theorem 3.1, we will need the preceding estimate of $\Gamma$ only in the case $|\alpha| \leq 3, \sigma \in\{0,1,2\}$. Finally we introduce the velocity part of a fundamental solution to the time-dependent Oseen system (1.1), putting

$$
\Lambda_{j k}(z, t):=\Gamma_{j k}\left(z-\tau t e_{1}, t\right) \text { for }(z, t) \in \mathfrak{B}, j, k \in\{1,2,3\} .
$$

Lemma 3.3 The relations $\Lambda \in C^{\infty}(\mathfrak{B})^{3 \times 3}$ and $\sum_{k=1}^{3} \partial z_{k} \Lambda_{j k}(z, t)=0$, as well as the equation $\left(\partial_{t} \Lambda-\Delta_{z} \Lambda+\partial z_{1} \Lambda\right)(z, t)=0$ are valid for $1 \leq j \leq 3, z \in \mathbb{R}^{3}, t \in(0, \infty)$.

Proof: Theorem 3.2.

We state some estimates of $\Lambda$.

Corollary 3.3 The inequality $\left|\partial_{z}^{\alpha} \partial_{t}^{\sigma} \Lambda(z, t)\right| \leq C(\tau) \sum_{\mu=1}^{2}\left(\left|z-\tau t e_{1}\right|^{2}+t\right)^{-(3+|\alpha|+\mu \sigma) / 2}$ holds for $(z, t) \in \mathbb{R}^{3} \times(0, \infty)$ and $(z, t) \in\left(\mathbb{R}^{3} \backslash\{0\}\right) \times\{0\}, \alpha \in \mathbb{N}_{0}^{3}$ with $|\alpha| \leq 3, \sigma \in$ $\{0,1,2\}$.

Let $K>0$. If $|z| \geq K$, then $\left|\partial_{z}^{\alpha} \partial_{t}^{\sigma} \Lambda(z, t)\right| \leq C(K, \tau) \sum_{\mu=1}^{2}(|z| \nu(z)+t)^{-(3+|\alpha|+\mu \sigma) / 2}$, else $\left|\partial_{z}^{\alpha} \partial_{t}^{\sigma} \Lambda(z, t)\right| \leq C(K, \tau) \sum_{\mu=1}^{2}\left(|z|^{2}+t\right)^{-(3+|\alpha|+\mu \sigma) / 2}$, for $z, t, \alpha, \sigma$ as before.

Proof: We have $\partial_{z}^{\alpha} \partial_{t}^{\sigma} \Lambda(z, t)=\sum_{j=0}^{\sigma}\left(\begin{array}{c}\sigma \\ j\end{array}\right)(-\tau)^{j} \partial_{x}^{\alpha} \partial x_{1}^{j} \partial_{r}^{\sigma-j} \Gamma(x, r)_{\mid x=z-\tau t e_{1}, r=t}$ for $z, t, \alpha$ and $\sigma$ as in the first part of the corollary. Thus the corollary follows from Theorem 3.2 and Lemma 3.2 .

Corollary 3.4 For $q \in(1, \infty), j, k \in\{1,2,3\}, t \in(0, \infty), \alpha \in \mathbb{N}_{0}^{3}$ with $|\alpha| \leq 3, \sigma \in$ $\{0,1,2\}$, we have

$$
\int_{\mathbb{R}^{3}}\left(\left|\partial_{x}^{\alpha} \partial_{t}^{\sigma} \mathfrak{H}(x, t)\right|^{q}+\left|\partial_{x}^{\alpha} \partial_{t}^{\sigma} \Gamma(x, t)\right|^{q}\right) d x \leq C t^{-(3+|\alpha|+2 \sigma) q / 2+3 / 2}<\infty,
$$

and if $\sigma \leq 1$,

$$
\int_{\mathbb{R}^{3}}\left(\left|\partial_{x}^{\alpha} \partial_{t}^{\sigma} \mathfrak{H}\left(x-\tau t e_{1}, t\right)\right|^{q}+\left|\partial_{x}^{\alpha} \partial_{t}^{\sigma} \Lambda(x, t)\right|^{q}\right) d x \leq C(\tau) \sum_{\mu=1}^{2} t^{-(3+|\alpha|+\mu \sigma) q / 2+3 / 2}<\infty .
$$

If $|\alpha|+\sigma>0$, the case $q=1$ is admitted as well.

Proof: Theorem 3.1 and the first estimate in Corollary 3.3. Use a change of variables to obtain $\int_{\mathbb{R}^{3}}\left(\left|z-\tau t e_{1}\right|^{2}+t\right)^{-\gamma} d z=\int_{\mathbb{R}^{3}}\left(|y|^{2}+t\right)^{-\gamma} d y$ for $\gamma \in(3, \infty), t \in(0, \infty)$.

In what follows, up to and including Corollary 3.6, we study functions of the form $(x, t) \mapsto$ $\int_{\mathbb{R}^{3}} \Lambda(x-y, t) U(y) d y$, with $U: \mathbb{R}^{3} \mapsto \mathbb{R}^{3}$ given.

Lemma 3.4 Let $q \in(1, \infty), \quad V \in W_{l o c}^{1,1}\left(\mathbb{R}^{3}\right)$ with $\nabla V \in L^{q}\left(\mathbb{R}^{3}\right)^{3}, \quad r \in(0, \infty)$ and $j \in\{1,2,3\}$. Then $\int_{\mathbb{R}^{3}} \sum_{k=1}^{3} \Gamma_{j k}(x-y, r) \partial_{k} V(y) d y=0$ for $x \in \mathbb{R}^{3}$, in particular $\int_{\mathbb{R}^{3}} \sum_{k=1}^{3} \Lambda_{j k}(x-y, r) \partial_{k} V(y) d y=0$ for such $x$. 
Proof: We adapt the proof of [11, Lemma 5.9], where we supposed $q<3$. Let $x \in \mathbb{R}^{3}$. We know from Corollary 3.4 that the function $y \mapsto \Gamma(x-y, r)\left(y \in \mathbb{R}^{3}\right)$ belongs to $L^{q^{\prime}}\left(\mathbb{R}^{3}\right)^{3 \times 3}$. Moreover, by Theorem 2.2 , there is a sequence $\left(\varphi_{n}\right)$ in $C_{0}^{\infty}\left(\mathbb{R}^{3}\right)$ such that $\left\|\nabla\left(\varphi_{n}-V\right)\right\|_{q} \rightarrow 0$. Thus

$$
\int_{\mathbb{R}^{3}} \sum_{k=1}^{3} \Gamma_{j k}(x-y, r) \partial_{k} V(y) d y=\lim _{n \rightarrow \infty} \int_{\mathbb{R}^{3}} \sum_{k=1}^{3} \Gamma_{j k}(x-y, r) \partial_{k} \varphi_{n}(y) d y .
$$

But the function $y \mapsto \Gamma(x-y, r)\left(y \in \mathbb{R}^{3}\right)$ is in particular of class $C^{1}$. By Theorem 3.2, we know that $\sum_{k=1}^{3} \partial y_{k} \Gamma_{j k}(x-y, r)=0$ for $y \in \mathbb{R}^{3}$. Therefore the first equation of the lemma follows from (3.2). The second follows from the first with $x$ replaced by $x-\tau r e_{1}$.

We further define

$$
\begin{gathered}
\widetilde{\Lambda}_{j k}:=\Lambda_{j k}-\mathfrak{H} \delta_{j k}, \text { that is, } \widetilde{\Lambda}_{j k}(z, t)=\int_{t}^{\infty} \partial z_{j} \partial z_{k} \mathfrak{H}\left(z-\tau t e_{1}, s\right) d s, \\
A_{j}(z, t):=\int_{t}^{\infty} \partial z_{j} \mathfrak{H}(z, s) d s \quad \text { for } 1 \leq j, k \leq 3, z \in \mathbb{R}^{3}, t \in(0, \infty) .
\end{gathered}
$$

Lemma 3.5 Let $j, k \in\{1,2,3\}$. Then $A_{j}(\cdot, t) \in C^{1}\left(\mathbb{R}^{3}\right)$ for $t \in(0, \infty)$ and the equation $\partial z_{k} A_{j}(z, t)=\int_{t}^{\infty} \partial z_{j} \partial z_{k} \mathfrak{H}(z, s) d s$ holds for $z \in \mathbb{R}^{3}, t>0$, hence $\partial z_{k} A_{j}\left(z-\tau t e_{1}, t\right)=$ $\widetilde{\Lambda}_{j k}(z, t)$ for $z, t$ as before.

Proof: We have $\left|\partial z_{k}^{\sigma} \partial z_{j} \mathfrak{H}(z, s)\right| \leq C s^{-(2+\sigma / 2)}$ for $z \in \mathbb{R}^{3}, s \in(0, \infty), \sigma \in\{0,1\}$ (Theorem 3.1). Thus the lemma follows with Lebesgue's theorem.

Lemma 3.6 Let $V \in W_{\text {loc }}^{1,1}\left(\bar{\Omega}^{c}\right)^{3}$ with div $V=0$ and $\nabla V \mid \Omega_{R} \in L^{1}\left(\Omega_{R}\right)^{9}$ for $R \in\left[S_{0}, \infty\right)$. (The number $S_{0}$ was fixed at the beginning of Section 2.) Suppose there is $m_{0} \in \mathbb{N}, p_{l} \in$ $(1, \infty), V^{(l)} \in L^{p_{l}}\left(\bar{\Omega}^{c}\right)^{3}$ for $1 \leq l \leq m_{0}$ such that $V=\sum_{l=1}^{m_{0}} V^{(l)}$. Take $x \in \bar{\Omega}^{c}, t \in(0, \infty)$. Then $\int_{\bar{\Omega}^{c}} \widetilde{\Lambda}(x-y, t) \cdot V(y) d y=\int_{\partial \Omega} A\left(x-y-\tau t e_{1}, t\right) n^{(\Omega)}(y) \cdot V(y) d o_{y}$.

Proof: Let $j \in\{1,2,3\}$. With Corollary 3.4 and Hölder's inequality, we get

$$
\begin{aligned}
& \int_{\bar{\Omega}^{c}} \int_{t}^{\infty}\left|\partial x_{k} \partial x_{j} \mathfrak{H}\left(x-y-\tau s e_{1}, s\right) V_{k}^{(l)}(y)\right| d s d y \\
& \leq \mathfrak{C}\left\|V^{(l)}\right\|_{p_{l}} \int_{t}^{\infty}\left(\int_{\mathbb{R}^{3}}\left|\partial x_{k} \partial x_{j} \mathfrak{H}\left(x-y-\tau s e_{1}, s\right)\right|^{p_{l}^{\prime}} d y\right)^{1 / p_{l}^{\prime}} d s \\
& \leq \mathfrak{C}\left\|V^{(l)}\right\|_{p_{l}} \int_{t}^{\infty} s^{-5 / 2+3 /\left(2 p_{l}^{\prime}\right)} d s \leq \mathfrak{C}\left\|V^{(l)}\right\|_{p_{l}} t^{-3 / 2+3 /\left(2 q_{1}^{\prime}\right)}
\end{aligned}
$$

for $k \in\{1,2,3\}, l \in\left\{1, \ldots, m_{0}\right\}$, where the last inequality holds because $-5 / 2+3 /\left(2 p_{l}^{\prime}\right)<$ -1 . Thus we may apply Fubini's theorem to obtain

$$
\int_{\bar{\Omega}^{c}} \sum_{k=1}^{3} \widetilde{\Lambda}_{j k}(x-y, t) V_{k}(y) d y=\int_{t}^{\infty} \int_{\bar{\Omega}^{c}} \sum_{k=1}^{3} \partial x_{k} \partial x_{j} \mathfrak{H}\left(x-y-\tau s e_{1}, s\right) V_{k}(y) d y d s
$$

By Corollary 3.4 and Theorem 3.1 the function $y \mapsto \partial x_{j} \mathfrak{H}\left(x-y-\tau s e_{1}, s\right)\left(y \in \mathbb{R}^{3}\right)$ belongs to $W^{1, q}\left(\mathbb{R}^{3}\right) \cap C^{\infty}\left(\mathbb{R}^{3}\right)$ for any $s \in(0, \infty), q \in(1, \infty)$. Thus, due to the assmptions on $V$, 
the function $\mathfrak{K}_{s}(y):=\partial x_{j} \mathfrak{H}\left(x-y-\tau s e_{1}, s\right) V(y)\left(y \in \bar{\Omega}^{c}\right)$ is in $W_{l o c}^{1,1}\left(\bar{\Omega}^{c}\right)^{3} \cap L^{1}\left(\bar{\Omega}^{c}\right)^{3}$ for any $s \in(0, \infty)$, with $\operatorname{div} \mathfrak{K}_{s} \in L^{1}\left(\bar{\Omega}^{c}\right)$ and $\nabla \mathfrak{K}_{s} \mid \Omega_{R} \in L^{1}\left(\Omega_{R}\right)^{9}$ for $R \in\left[S_{0}, \infty\right)$. This fact, the equation $\operatorname{div} V=0$ and Lemma 2.2 yield that

$$
\begin{aligned}
& \int_{\bar{\Omega}^{c}} \sum_{k=1}^{3} \partial x_{k} \partial x_{j} \mathfrak{H}\left(x-y-\tau s e_{1}, s\right) V_{k}(y) d y \\
& =\int_{\partial \Omega} \partial x_{j} \mathfrak{H}\left(x-y-\tau s e_{1}, s\right) n^{(\Omega)}(y) \cdot V(y) d o_{y} \quad \text { for } s \in(0, \infty) .
\end{aligned}
$$

But $\operatorname{dist}(x, \partial \Omega)>0$, so by Lemma 3.2 with $K=\operatorname{dist}(x, \partial \Omega)$ and by Theorem 3.1 we get $\left|\partial x_{j} \mathfrak{H}\left(x-y-\tau s e_{1}, s\right)\right| \leq \mathfrak{C}(K)(\delta+s)^{-2}$ for $y \in \partial \Omega, s \in(0, \infty)$. As a consequence

$$
\begin{aligned}
& \int_{t}^{\infty} \int_{\partial \Omega}\left|\partial x_{j} \mathfrak{H}\left(x-y-\tau s e_{1}, s\right) n^{(\Omega)}(y) \cdot V(y)\right| d o_{y} d s \\
& \leq \mathfrak{C}(K) \int_{t}^{\infty}(\delta+s)^{-2} d s \int_{\partial \Omega}|V(y)| d o_{y}<\infty .
\end{aligned}
$$

So the integral $\int_{t}^{\infty} \int_{\partial \Omega} \partial x_{j} \mathfrak{H}\left(x-y-\tau s e_{1}, s\right) n^{(\Omega)}(y) \cdot V(y) d o_{y} d s$ may be transformed via Fubini's theorem. The lemma then follows from (3.6) and (3.7).

Lemma 3.7 Let $q \in(1, \infty), V \in L_{\sigma}^{q}\left(\mathbb{R}^{3}\right), r \in(0, \infty)$. Then $\int_{\mathbb{R}^{3}} \nabla_{x} A_{j}(x-y, r) \cdot V(y) d y=$ 0 for $x \in \mathbb{R}^{3}, 1 \leq j \leq 3$, in particular $\int_{\mathbb{R}^{3}} \widetilde{\Lambda}(x-y, r) \cdot V(y) d y=0$ for $x \in \mathbb{R}^{3}$.

Proof: See [11, Lemma 5.10] for the first equation. The second follows from the first with $x$ replaced by $x-\tau r e_{1}$.

Now we may introduce the first of our potential functions.

Corollary 3.5 Let $A \subset \mathbb{R}^{3}$ be measurable, $q \in[1, \infty), V \in L^{q}(A)^{3}, \widetilde{V}$ the zero extension of $V$ to $\mathbb{R}^{3}$. Then

$$
\int_{\mathbb{R}^{3}}\left|\partial_{x}^{\alpha} \partial_{t}^{\sigma} \Lambda(x-y, t) \widetilde{V}(y)\right| d y \leq C(\tau, q) \sum_{\mu=1}^{2} t^{-(3+|\alpha|+\sigma \mu) / 2+3 /\left(2 q^{\prime}\right)}\|V\|_{q}
$$

for $\alpha \in \mathbb{N}_{0}^{3}, \sigma \in\{0,1\}$ with $|\alpha|+2 \sigma \leq 2, x \in \mathbb{R}^{3}, t \in(0, \infty)$. Define

$$
\mathfrak{I}^{(\tau)}(V)(x, t):=\int_{\mathbb{R}^{3}} \Lambda(x-y, t) \cdot \widetilde{V}(y) d y \quad \text { for } x \in \mathbb{R}^{3}, t \in(0, \infty) .
$$

For $\alpha, \sigma, x, t$ as in (3.8), the derivative $\partial_{x}^{\alpha} \partial_{t}^{\sigma} \mathfrak{I}^{(\tau)}(V)(x, t)$ exists and equals the integral $\int_{\mathbb{R}^{3}} \partial_{x}^{\alpha} \partial_{t}^{\sigma} \Lambda(x-y, t) \cdot \widetilde{V}(y) d y$, and is a continuous function of $(x, t) \in \mathbb{R}^{3} \times(0, \infty)$. With the abbreviation $u:=\mathfrak{I}^{(\tau)}(V)$, the equations div $u=0, \partial_{t} u-\Delta_{x} u+\tau \partial x_{1} u=0$ hold. If $q>1$, then for $x \in \mathbb{R}^{3}, t>0$,

$$
\mathfrak{I}^{(\tau)}(V)(x, t)=\int_{\mathbb{R}^{3}} \mathfrak{H}\left(x-y-\tau t e_{1}, t\right) \cdot P_{q}(\widetilde{V})(y) d y
$$

(see Theorem 2.3 for the definition of the operator $P_{q}$ ) and $\left\|\mathfrak{I}^{(\tau)}(V)(t)\right\|_{q} \leq C(q, \tau)\|V\|_{q}$. If $m_{0} \in \mathbb{N}, p_{l} \in[1, \infty), V^{(l)} \in L^{p_{l}}(A)^{3}$ for $1 \leq l \leq m_{0}$, put $\mathfrak{I}^{(\tau)}\left(\sum_{l=1}^{m_{0}} V^{(l)}\right):=$ $\sum_{l=1}^{m_{0}} \mathfrak{I}^{(\tau)}\left(V^{(l)}\right)$. 
Proof: We have $\left|\partial_{x}^{\alpha} \partial_{t}^{\sigma} \Lambda(x-y, t)\right| \leq C(\tau) \sum_{\mu=1}^{2} t^{-(3+|\alpha|+\sigma \mu) / 2}$ for $\alpha, \sigma, x, t$ as in (3.8), as follows from the first inequality in Corollary 3.3. If $q=1$, the preceding estimate together with Lebesgue's theorem and Lemma 3.3 implies (3.8) as well as the second statement of the corollary, pertaining to differentiability and continuity.

Now suppose that $q>1$. Then inequality (3.8) follows from Corollary 3.4 and Hölder's inequality. As for the second claim of the corollary, we take $R>0$ and differentiate $\mathfrak{I}^{(\tau)}(V) \mid B_{R}$ after splitting the domain of intergration $\mathbb{R}^{3}$ in the definition of $\mathfrak{I}^{(\tau)}(V)$ into the parts $B_{2 R}$ and $\mathbb{R}^{3} \backslash B_{2 R}$. The integral over $B_{2 R}$ may be handled in the same way as the integral over $\mathbb{R}^{3}$ in the case $q=1$ because $\widetilde{V} \mid B_{2 R} \in L^{1}\left(B_{2 R}\right)^{3}$. As concerns the integral over $B_{2 R}^{c}$, we note that $\left|\partial_{x}^{\alpha} \partial_{t}^{\sigma} \Lambda(x-y, t)\right| \leq C(\tau, R) \sum_{\mu=1}^{2}(|y| \nu(y))^{-(3+|\alpha|+\sigma \mu) / 2}$ for $x \in$ $B_{R}, y \in B_{2 R}^{c}$ and for $t, \alpha, \sigma$ as in (3.8), as follows from the first estimate in Corollary 3.3 and Corollary 3.1. Since by Corollary 3.2, the function $\sum_{\mu=1}^{2}(|y| \nu(y))^{-(3+|\alpha|+\sigma \mu) q^{\prime} / 2}(y \in$ $\left.B_{2 R}^{c}\right)$ is integrable if $|\alpha|+\sigma \geq 1$, the assumption $V \in L^{q}(A)^{3}$, Hölder's inequality and Lebesgue's theorem yield that the derivative $\partial_{x}^{\alpha} \partial_{t}^{\sigma} \mathfrak{I}^{(\tau)}\left(\chi_{B_{2 R}^{c}} V\right)(x, t)$ exists for $x \in B_{R}, t \in$ $(0, \infty), \alpha \in \mathbb{N}_{0}^{3}, \sigma \in\{0,1\}$ with $0<|\alpha|+2 \sigma \leq 2$, and it equals $\int_{B_{2 R}^{c}} \partial_{x}^{\alpha} \partial_{t}^{\sigma} \Lambda(x-y, t) \widetilde{V}(y) d y$ and is continuous as a function of $x \in B_{R}, t \in(0, \infty)$. Altogether we see that the second assertion of the corollary, pertaining to derivatives of $\mathfrak{I}^{(\tau)}(V)$, holds true. This assertion and Lemma 3.3 yield the differential equations stated in the corollary. The equation $V=P_{q}(\widetilde{V})+\nabla G_{q}(\widetilde{V})$ (Theorem 2.3), Lemma 3.4 and 3.7 imply (3.9). The $L^{q}$-estimate at the end of the corollary follows from Young's inequality, Theorem 2.3 and the equation in Theorem 3.1.

Theorem 3.3 Let $q \in(1, \infty), T_{0} \in(0, \infty], t \in\left(0, T_{0}\right)$ and $u$ a function belonging to $W_{\text {loc }}^{1,1}\left(\left[0, T_{0}\right), L^{q}\left(\mathbb{R}^{3}\right)^{3}\right)$ and to $C^{0}\left(\left[0, T_{0}\right), L^{q}\left(\mathbb{R}^{3}\right)^{3}\right)$. Then

$$
\int_{\mathbb{R}^{3}}\left|\int_{\mathbb{R}^{3}} \mathfrak{H}\left(x-y-\tau \epsilon e_{1}, \epsilon\right) u(y, t-\epsilon) d y-u(x, t)\right|^{q} d x \rightarrow 0(\epsilon \downarrow 0) .
$$

Proof: Let $\epsilon \in(0, t)$ and $x \in \mathbb{R}^{3}$. Then $\int_{\mathbb{R}^{3}} \mathfrak{H}\left(x-y-\tau \epsilon e_{1}, \epsilon\right)^{r} d y \leq C(\tau, r) \epsilon^{-3(r-1) / 2}<$ $\infty$ for $r \in(1, \infty)$ by Corollary 3.4. Thus, taking $r=q^{\prime}$, we may define a constant function $G:(t-\epsilon, t) \mapsto\left[L^{q}\left(\mathbb{R}^{3}\right)^{3}\right]^{\prime}$ by setting $G(s)(V):=\int_{\mathbb{R}^{3}} \mathfrak{H}\left(x-y-\epsilon \tau e_{1}, \epsilon\right) V(y) d y$ for $s \in(t-\epsilon, t), V \in L^{q}\left(\mathbb{R}^{3}\right)^{3}$. Since it is constant, this function obviously belongs to $W^{1,1}\left(t-\epsilon, t,\left[L^{q}\left(\mathbb{R}^{3}\right)^{3}\right]^{\prime}\right)$ and to $C^{0}\left([t-\epsilon, t],\left[L^{q}\left(\mathbb{R}^{3}\right)^{3}\right]^{\prime}\right)$. This observation, the equation $G^{\prime}=0$ and the assumptions on $u$ allow us to conclude with Lemma 2.5 that $\int_{t-\epsilon}^{\epsilon} G(t)\left(u^{\prime}(t)\right) d t=G(t)(u(t))-G(t-\epsilon)(u(t-\epsilon))$. Due to the definition of $G$, this equation translates into

$\int_{\mathbb{R}^{3}} \mathfrak{H}\left(x-y-\tau \epsilon e_{1}, \epsilon\right)(-u(y, t-\epsilon)+u(y, t)) d y=\int_{t-\epsilon}^{t} \int_{\mathbb{R}^{3}} \mathfrak{H}\left(x-y-\tau \epsilon e_{1}, \epsilon\right) u^{\prime}(y, s) d y d s$ for $\epsilon \in(0, t)$. Since $\int_{\mathbb{R}^{3}} \mathfrak{H}\left(x-y-\tau \epsilon e_{1}, \epsilon\right)=1$ for $\epsilon \in(0, \infty)$ (Theorem 3.1), Minkowski's and Young's inequality (Lemma 2.1, Theorem 2.1) allow to conclude that

$$
\left(\int_{\mathbb{R}^{3}}\left|\int_{\mathbb{R}^{3}} \mathfrak{H}\left(x-y-\tau \epsilon e_{1}, \epsilon\right)(u(y, t-\epsilon)-u(y, t)) d y\right|^{q} d x\right)^{1 / q} \leq \mathfrak{C} \int_{t-\epsilon}^{t}\left\|u^{\prime}(r)\right\|_{q} d r .
$$

Let $\kappa \in(0, \infty)$. Since $u \in W_{l o c}^{1,1}\left(\left[0, T_{0}\right), L^{q}\left(\mathbb{R}^{3}\right)^{3}\right)$, we may thus choose $\epsilon_{1} \in(0, t)$ so small that the right-hand side of the preceding inequality, and hence the left-hand one, too, 
is bounded by $\kappa / 4$ for any $\epsilon \in\left(0, \epsilon_{1}\right]$. As in the proof of [15, Theorem 4.1], we choose $\psi \in C_{0}^{\infty}\left(\mathbb{R}^{3}\right)^{3}$ with $\|u(t)-\psi\|_{q} \leq \kappa / 4$. Again since $\int_{\mathbb{R}^{3}} \mathfrak{H}\left(x-y-\tau \epsilon e_{1}, \epsilon\right) d y=1$ (Theorem 3.1 ), we then obtain by Young's inequality that

$$
\left(\int_{\mathbb{R}^{3}}\left(\int_{\mathbb{R}^{3}} \mathfrak{H}\left(x-y-\tau \epsilon e_{1}, \epsilon\right)|u(y, t)-\psi(y)| d y\right)^{q} d x\right)^{1 / q} \leq \mathfrak{C}\|u(t)-\psi\|_{q} \leq \kappa / 4
$$

for $\epsilon \in(0, \infty)$. Next we set $M(x, \epsilon):=\int_{\mathbb{R}^{3}} \mathfrak{H}\left(x-y-\tau \epsilon e_{1}, \epsilon\right) \psi(y) d y$ for $x \in \mathbb{R}^{3}, \epsilon>0$. As explained in the proof of [10, Theorem 2.16], the term $M(x, \epsilon)$ tends to $\psi(x)$ for $\epsilon \downarrow 0$, uniformly in $x \in \mathbb{R}^{3}$. Choose $S \in(0, \infty)$ with $\operatorname{supp}(\psi) \subset B_{S / 2}$. Then we may conclude there is $\epsilon_{2}$ such that $\left\|M(\epsilon)-\psi \mid B_{S}\right\|_{q} \leq \kappa / 4$ for $\epsilon \in\left(0, \epsilon_{2}\right]$. Since $\mathfrak{H} \in C^{\infty}(\mathfrak{B})$ (Theorem $3.1), \mathfrak{H}(z, 0)=0$ for $z \in \mathbb{R}^{3} \backslash\{0\}$, and $|x-y| \geq|x|-|y| \geq S / 2>0$ for $x \in B_{S}^{c}, y \in B_{S / 2}$, we find that $H\left(x-y-\tau \delta e_{1}, \delta\right) \rightarrow 0(\delta \downarrow 0)$ for such $x$ and $y$. As a consequence, for $x \in B_{S}^{c}, y \in B_{S / 2}, \epsilon>0$,

$$
\begin{aligned}
& \mathfrak{H}\left(x-y-\tau \epsilon e_{1}, \epsilon\right)=\lim _{\delta \downarrow 0}\left(\mathfrak{H}\left(x-y-\tau \epsilon e_{1}, \epsilon\right)-\mathfrak{H}\left(x-y-\tau \delta e_{1}, \delta\right)\right) \\
& =\lim _{\delta \downarrow 0} \int_{0}^{1}\left(\partial_{t} \mathfrak{H}-\tau \partial x_{1} \mathfrak{H}\right)\left(x-y-\tau t e_{1}, t\right)_{\mid t=\delta+\vartheta(\epsilon-\delta)} d \vartheta(\epsilon-\delta) .
\end{aligned}
$$

Therefore by Theorem 3.1 and Corollary 3.1,

$$
\mathfrak{H}\left(x-y-\tau \epsilon e_{1}, \epsilon\right) \leq \mathfrak{C} \limsup _{\delta \downarrow 0} \sum_{\mu=0}^{1} \int_{0}^{1}(|x| \nu(x)+\delta+\vartheta(\epsilon-\delta))^{-2-\mu / 2} d \vartheta(\epsilon-\delta),
$$

hence $0 \leq \mathfrak{H}\left(x-y-\tau \epsilon e_{1}, \epsilon\right) \leq \mathfrak{C}(|x| \mu(x))^{-2} \epsilon$. Recalling that $\operatorname{supp}(\psi) \subset B_{S / 2}$, we thus get

$$
\left\|M(\epsilon)-\psi\left|B_{S}^{c}\left\|_{q}=\right\| M(\epsilon)\right| B_{S}^{c}\right\|_{q} \leq \mathfrak{C} \epsilon\left(\int_{B_{S}^{c}}(|x| \mu(x))^{-2 q^{\prime}} d x\right)^{1 / q^{\prime}}\|\psi\|_{q} \leq \mathfrak{C} \epsilon\|\psi\|_{q}
$$

for $\epsilon>0$, where the last inequality is a consequence of Corollary 3.2. Thus we may choose $\epsilon_{3}>0$ with $\left\|M(\epsilon)-\psi \mid B_{S}^{c}\right\|_{q} \leq \kappa / 4$ for $\epsilon \in\left(0, \epsilon_{3}\right]$. The preceding inequalities imply (3.10).

Corollary 3.6 Let $q \in(1, \infty), V \in L^{q}\left(\mathbb{R}^{3}\right)^{3}$. Put $\mathfrak{I}^{(\tau)}(V)(0):=P_{q}(V)$, with $P_{q}$ from Theorem 2.3. Then $\mathfrak{I}^{(\tau)}(V) \in C^{0}\left([0, \infty), L^{q}\left(\mathbb{R}^{3}\right)^{3}\right)$ and $\left\|\mathfrak{I}^{(\tau)}(V)(b)-\mathfrak{I}^{(\tau)}(V)(a)\right\|_{2} \leq$ $C(\tau)\left(a^{-1}+a^{-1 / 2}\right)\|V\|_{q}(b-a)$ for $a, b \in(0, \infty)$ with $a<b$.

Proof: For $a, b$ as above, we find with Corollary 3.5, 3.4 and Young's inequality that

$$
\begin{aligned}
& \left\|\mathfrak{I}^{(\tau)}(V)(b)-\mathfrak{I}^{(\tau)}(V)(a)\right\|_{q} \leq \int_{\mathbb{R}^{3}}\left|\int_{0}^{1} \partial_{t} \Lambda(z, t)_{\mid t=a+\vartheta(b-a)} d \vartheta\right| d z\|V\|_{q}(b-a) \\
& \leq C(\tau) \int_{0}^{1} \sum_{\mu=1}^{2}(a+\vartheta(b-a))^{-\mu / 2} d \vartheta\|V\|_{q}(b-a) \leq C(\tau)\left(a^{-1}+a^{-1 / 2}\right)\|V\|_{q}(b-a) .
\end{aligned}
$$

Thus the inequality at the end of the corollary is proved. In particular, this settles continuity of $\mathfrak{I}^{(\tau)}(V)$ on $(0, \infty)$. As for $t=0$, we recall that by Corollary 3.5 , we have 
$\mathfrak{I}^{(\tau)}(V)(x, t)=\int_{\mathbb{R}^{3}} \mathfrak{H}\left(x-y-\tau t e_{1}, t\right) P_{q}(V)(y) d y$ for $x \in \mathbb{R}^{3}, t>0$. Thus by Theorem $3.3,\left\|\mathfrak{I}^{(\tau)}(V)(\epsilon)-P_{q}(V)\right\|_{q} \rightarrow 0(\epsilon \downarrow 0)$. So $\mathfrak{I}^{(\tau)}(V)$ is continuous also in $t=0$.

We turn to the definition of a second potential function, beginning with a technical tool.

Theorem 3.4 Let $p, q \in[1, \infty), r \in(1, \infty), s \in[1, \infty)$ with $q<p, s \leq r$. Let $\alpha \in \mathbb{N}_{0}^{3}$ with $|\alpha| \leq 1$. Suppose that $1-|\alpha| / 2+3(1 / p-1 / q) / 2>1 / s-1 / r$. Let $h \in$ $L^{s}\left(0, \infty, L^{q}\left(\mathbb{R}^{3}\right)^{3}\right)$ and $M \in(0, \infty)$. Then

$$
\begin{aligned}
& \left(\int_{0}^{\infty}\left(\int_{\mathbb{R}^{3}}\left[\int_{0}^{\infty} \int_{\mathbb{R}^{3}}\left|\chi_{(0, M)}(t-\sigma) \partial_{x}^{\alpha} \Lambda(x-y, t-\sigma) \cdot h(y, \sigma)\right| d y d \sigma\right]^{p} d x\right)^{r / p} d t\right)^{1 / r} \\
& \leq C(\tau, p, q, r, s) M^{1-|\alpha| / 2+3(1 / p-1 / q) / 2-1 / s+1 / r}\|h\|_{q, s ; \infty} .
\end{aligned}
$$

Proof: See [14, Theorem 2.8].

Lemma 3.8 Let $T_{0} \in(0, \underset{\sim}{\infty}], A \subset \mathbb{R}^{3}$ measurable, $q \in[1, \infty)$ and $f$ a function from $L_{\text {loc }}^{1}\left(\left[0, T_{0}\right), L^{q}(A)^{3}\right)$. Let $\widetilde{f}$ denote the zero extension of $f$ to $\mathbb{R}^{3} \times(0, \infty)$. Then the integral $\int_{\mathbb{R}^{3}}\left|\partial_{x}^{\alpha} \Lambda(x-y, t-\sigma) \cdot \widetilde{f}(y, \sigma)\right| d y$ is finite for any $x \in \mathbb{R}^{3}, t \in(0, \infty), \sigma \in$ $(0, t), \alpha \in \mathbb{N}_{0}^{3}$ with $|\alpha| \leq 1$. Moreover, for a. e. $t \in(0, \infty)$ and for $\alpha$ as before, the integral $\int_{0}^{t} \int_{\mathbb{R}^{3}}\left|\partial_{x}^{\alpha} \Lambda(x-y, t-\sigma) \cdot \widetilde{f}(y, \sigma)\right| d y d \sigma$ is finite as well for a. e. $x \in \mathbb{R}^{3}$. Due to this latter fact, we may define

$$
\mathfrak{R}^{(\tau)}(f)(x, t):=\int_{0}^{t} \int_{\mathbb{R}^{3}} \Lambda(x-y, t-\sigma) \cdot \widetilde{f}(y, \sigma) d y d \sigma
$$

for such $t$ and $x$. The relation $\mathfrak{R}^{(\tau)}(f)(t) \in W_{\text {loc }}^{1,1}\left(\mathbb{R}^{3}\right)^{3}$ holds for a. e. $t \in(0, \infty)$, and for such $t, \partial x_{l} \mathfrak{R}^{(\tau)}(f)(t)(x)=\int_{0}^{t} \int_{\mathbb{R}^{3}} \partial x_{l} \Lambda(x-y, t-\sigma) \cdot \widetilde{f}(y, \sigma) d y d \sigma$ for $l \in\{1,2,3\}$ and $a$. e. $x \in \mathbb{R}^{3}$.

Proof: The first claim of the lemma follows from (3.8) Choose $p \in(q, \infty)$ and $r \in$ $(1, \infty)$ so close to $q$ and 1 , respectively, that $1 / 2+3(1 / p-1 / q) / 2>1-1 / r$. Then for $T \in(0, \infty), \alpha \in \mathbb{N}_{0}^{3}$ with $|\alpha| \leq 1$, Theorem 3.4 with $M=T, s=1$ yields that $\int_{0}^{T}\left(\int_{\mathbb{R}^{3}}\left[\int_{0}^{t} \int_{\mathbb{R}^{3}}\left|\partial_{x}^{\alpha} \Lambda(x-y, t-\sigma) \cdot \widetilde{f}(y, \sigma)\right| d y d \sigma\right]^{p} d x\right)^{r / p} d t<\infty$. This implies the lemma.

Theorem 3.4 remains valid if $r=\infty$, with obvious modifications of the inequality stated in it; see [14, Theorem 2.8]. However, it is mute if $r=\infty, s=1$ because then there is no $p \in(q, \infty)$ such that $1+3(1 / p-1 / q) / 2>1 / s-1 / r$. But this case will be relevant in what follows, and it may be handled by referring to our results on $\mathfrak{I}^{(\tau)}(V)$. Here are the details. Recall the first claim in Lemma 3.8.

Corollary 3.7 Let $T_{0} \in(0, \infty], A \subset \mathbb{R}^{3}$ measurable, $q \in(1, \infty), f$ a function belonging to $L_{\text {loc }}^{1}\left(\left[0, T_{0}\right), L^{q}(A)^{3}\right)$ and $\widetilde{f}$ the zero extension of $f$ to $\mathbb{R}^{3} \times(0, \infty)$. Then

$$
\left(\int_{\mathbb{R}^{3}}\left[\int_{0}^{t}\left|\int_{\mathbb{R}^{3}} \Lambda(x-y, t-s) \cdot \widetilde{f}(y, s) d y\right| d s\right]^{q} d x\right)^{1 / q} \leq C(q)\left\|\widetilde{f} \mid \mathbb{R}^{3} \times(0, t)\right\|_{q, 1 ; t}
$$

for $t \in(0, \infty)$. In particular the integral $\int_{0}^{t}\left|\int_{\mathbb{R}^{3}} \Lambda(x-y, t-s) \cdot \widetilde{f}(y, s) d y\right| d s$ is finite for any $t \in(0, \infty)$ and for a. e. $x \in \mathbb{R}^{3}$. Thus the function $\mathfrak{R}^{(\tau)}(f)$ is well defined for 
any $t \in(0, \infty)$ (instead of only for a. e. $t \in(0, \infty)$ ) and for a. e. $x \in \mathbb{R}^{3}$. Moreover $\left\|\mathfrak{R}^{(\tau)}(f)(t)\right\|_{q} \leq C(q)\left\|\widetilde{f} \mid \mathbb{R}^{3} \times(0, t)\right\|_{q, 1 ; t}$ for $t>0$.

Let $m_{0} \in \mathbb{N}, p_{l} \in(1, \infty)$ and $f^{(l)} \in L_{l o c}^{1}\left(\left[0, T_{0}\right), L^{p_{l}}(A)^{3}\right)$ for $1 \leq l \leq m_{0}$. Then define $\mathfrak{R}^{(\tau)}\left(\sum_{j=1}^{m_{0}} f^{(l)}\right):=\sum_{j=1}^{m_{0}} \mathfrak{R}\left(f^{(l)}\right)$.

Proof: Let $t \in(0, \infty)$, and denote the left-hand side of (3.11) by $\mathfrak{A}(t)$. Then, by Theorem 2.1 and Corollary 3.5, we have $\mathfrak{A}(t) \leq \int_{0}^{t}\left\|\mathfrak{I}^{(\tau)}(f(s))(t-s)\right\|_{q} d s \leq \int_{0}^{t} C(q)\|f(s)\|_{q} d s$, and thus $\mathfrak{A}(t) \leq C(q)\left\|\widetilde{f} \mid \mathbb{R}^{3} \times(0, t)\right\|_{q, 1 ; t}$. This proves the corollary.

We define a third potential function:

Lemma 3.9 Let $q \in[1, \infty], T_{0} \in(0, \underset{\widetilde{\phi}}{\infty}], A \subset \mathbb{R}^{3}$ open and bounded, with Lipschitz boundary, $\phi \in L_{l o c}^{1}\left(\left[0, T_{0}\right), L^{q}(\partial A)^{3}\right)$, $\widetilde{\phi}$ the zero extension of $\phi$ to $\partial A \times(0, \infty)$. For $t \in(0, \infty), x \in \mathbb{R}^{3} \backslash \partial A, \alpha \in \mathbb{N}_{0}^{3}$, the term $\left|\partial_{x}^{\alpha} \Lambda(x-y, t-s) \cdot \widetilde{\phi}(y, s)\right|$ is integrable as a function of $(y, s) \in \partial A \times(0, t)$. Define $\mathfrak{V}^{(\tau)}(\phi):=\mathfrak{V}^{(\tau, A)}(\phi):\left(\mathbb{R}^{3} \backslash \partial A\right) \times(0, \infty) \mapsto \mathbb{R}^{3}$ by

$$
\mathfrak{V}^{(\tau)}(\phi)(x, t):=\int_{0}^{t} \int_{\partial A} \Lambda(x-y, t-s) \cdot \widetilde{\phi}(y, s) d o_{y} d s \quad \text { for } x \in \mathbb{R}^{3} \backslash \partial A, t \in(0, \infty) .
$$

Then, for any $t \in(0, \infty)$, the integral $\int_{0}^{t} \int_{\partial A} \Lambda(x-y, t-s) \cdot \widetilde{\phi}(y, s) d o_{y} d s$ as a function of $x \in$ $\mathbb{R}^{3} \backslash A$ belongs to $C^{\infty}\left(\mathbb{R}^{3} \backslash A\right)^{3}$, and $\partial_{x}^{\alpha} \mathfrak{V}^{(\tau)}(\phi)(x, t)=\int_{0}^{t} \int_{\partial A} \partial_{x}^{\alpha} \Lambda(x-y, t-s) \cdot \widetilde{\phi}(y, s) d o_{y} d s$ for $\alpha \in \mathbb{N}_{0}^{3}, x \in \mathbb{R}^{3} \backslash A$.

Proof: The function $\Lambda$ is $C^{\infty}$ on $\mathbb{R}^{3} \times(0, \infty)$ (Lemma 3.3), so the lemma follows with Lebesgue's theorem.

In view of introducing another kernel function - a truncated version of $\Lambda$-, we abbreviate $A:=A_{R_{1}, S_{0}}$, and define

$$
M_{\varphi_{0}}(x, y, r):=\left(\sum_{k=1}^{3} \partial_{k} \varphi_{0}(y) \Lambda_{j k}(x-y, r)\right)_{1 \leq j \leq 3} \text { for } x \in B_{R_{0}}^{c}, y \in A, r \in[0, \infty) .
$$

(Recall that the numbers $S_{0}, R_{0}, R_{1}$ and the function $\varphi_{0}$ were fixed at the beginning of Section 2, with $S_{0}<R_{0}$ and $R_{1}:=\left(S_{0}+R_{0}\right) / 2$.)

Lemma 3.10 Let $x \in B_{R_{0}}^{c}, r \in[0, \infty), \sigma \in\{0,1\}, \alpha \in \mathbb{N}_{0}^{3}$ with $|\alpha| \leq 1$. Then the function $\partial_{x}^{\alpha} \partial_{r}^{\sigma} M_{\varphi_{0}}(x, \cdot, r)$ belongs to $C_{0}^{\infty}(A)^{3}$, and $\int_{A} \partial_{x}^{\alpha} \partial_{r}^{\sigma} M_{\varphi_{0}}(x, y, r) d y=0$.

Proof: Use Lemma 3.3; compare the proof of [15, Lemma 2.13].

Due to Lemma 3.10 and Theorem 2.4, we may define a function $\mathfrak{G}:=\mathfrak{G}_{R_{0}, S_{0}, \varphi_{0}}: B_{R_{0}}^{c} \times$ $B_{R_{1}} \times[0, \infty) \mapsto \mathbb{R}^{3 \times 3}$ by setting

$$
\mathfrak{G}(x, y, r):=\left(\varphi_{0}(y) \Lambda_{j k}(x, y, r)-\mathfrak{D}\left[M_{\varphi_{0}, j}(x, \cdot, r)\right]_{k}(y)\right)_{1 \leq j, k \leq 3}
$$

for $x \in B_{R_{0}}^{c}, y \in B_{R_{1}}, r \in[0, \infty)$, where the operator $\mathfrak{D}:=\mathfrak{D}_{R_{1}, S_{0}}$ was introduced in Theorem 2.4. In the rest of this section, we will always write $\mathfrak{G}$ instead of $\mathfrak{G}_{R_{0}, S_{0}, \varphi_{0}}$.

Lemma 3.11 Let $x \in B_{R_{0}}^{c}, r \in[0, \infty)$. Then $\mathfrak{G}(x, \cdot, r) \in C_{0}^{\infty}\left(B_{R_{1}}\right)^{3 \times 3}$, the equation $\sum_{k=1}^{3} \partial y_{k} \mathfrak{G}_{j k}(x, y, r)=0$ holds for $1 \leq j \leq 3, y \in B_{R_{1}}$, and $\mathfrak{G}(x, y, r)=\Lambda(x-y, r)$ for $y \in B_{S_{0}+\left(R_{0}-S_{0}\right) / 4}$. 
Proof: Lemma 3.3, Theorem 2.4.

Lemma 3.12 Let $x \in B_{R_{0}}^{c}, q \in(1, \infty)$. Then the mapping $r \mapsto \mathfrak{G}(x, \cdot, r)(r \in[0, \infty))$ belongs to $C^{1}\left([0, \infty), W^{1, q}\left(B_{R_{1}}\right)^{3 \times 3}\right)$. Thus a function $G^{\prime} \in C^{0}\left([0, \infty), W^{1, q}\left(B_{R_{1}}\right)^{3 \times 3}\right)$ may be defined by the condition $\left\|(\mathfrak{G}(x, \cdot, r+h)-\mathfrak{G}(x, \cdot, r)) / h-G^{\prime}(r)\right\|_{1, q} \rightarrow 0(h \rightarrow 0)$ for $r \in[0, \infty)$. We write $\partial_{r} \mathfrak{G}(x, y, r)$ instead of $G^{\prime}(r)(y)\left(r \in[0, \infty), y \in B_{R_{1}}\right)$. Then

$$
\partial_{r} \mathfrak{G}_{j k}(x, y, r)=\varphi_{0}(y) \partial_{r} \Lambda_{j k}(x, y, r)-\mathfrak{D}\left[\partial_{r} M_{\varphi_{0}, j}(x, \cdot, r)\right]_{k}(y)
$$

for $y \in B_{R_{1}}, r \in[0, \infty), 1 \leq j, k \leq 3$, with $\mathfrak{D}:=\mathfrak{D}_{R_{1}, S_{0}}$ from Theorem 2.4.

Proof: Recall that we have set $A:=A_{R_{1}, S_{0}}$ in the passage preceding (3.12). Let $j, k \in$ $\{1,2,3\}, r \in[0, \infty), h \in \mathbb{R} \backslash\{0\}$ with $r+h \in[0, \infty)$. Set $R^{\prime}:=\left(R_{0}-S_{0}\right) / 2$, and put $Z(y):=\left(M_{\varphi_{0}}(x, y, r+h)-M_{\varphi_{0}}(x, y, r)\right) / h-\partial_{r} M_{\varphi_{0}}(x, y, r)$ for $y \in A$. Then Lemma 3.10 , Theorem 2.4 and the choice of $\varphi_{0}$ and $x$ yield that $\left\|\mathfrak{D}\left(Z_{j}\right)_{k}\right\|_{1, q}$ is bounded by $\mathfrak{C}\left\|Z_{j}\right\|_{q}$, and therefore (Theorem 2.1) by

$$
\mathfrak{C}|h| \sum_{l=1}^{3} \int_{0}^{1} \int_{0}^{1}\left(\int_{B_{R_{1}}} \chi_{\left(R^{\prime}, \infty\right)}(|x-y|)\left|\partial_{t}^{2} \Lambda_{j l}(x-y, t)_{\mid t=r+\vartheta \gamma h}\right|^{q} d y\right)^{1 / q} d \gamma d \vartheta .
$$

Put $\left.\widetilde{Z}(y):=\varphi_{0}(y)\left(\Lambda_{j k}(x, y, r+h)-\Lambda_{j k}(x, y, r)\right) / h-\partial_{r} \Lambda(x, y, r)_{j k}\right)_{1 \leq j, k \leq 3}$ for $y \in B_{R_{1}}$. By Theorem 2.1, Lemma 3.3 and the choice of $\varphi_{0}$ and $x$, the term $\left\|\widetilde{Z}_{j k}\right\|_{1, q}$ is bounded by

$$
\mathfrak{C}|h| \sum_{\alpha \in \mathbb{N}_{0}^{3},|\alpha| \leq 1} \int_{0}^{1} \int_{0}^{1}\left(\int_{B_{R_{1}}} \chi_{\left(R^{\prime}, \infty\right)}(|x-y|)\left|\partial_{t}^{2} \partial_{y}^{\alpha} \Lambda_{j k}(x-y, t)_{\mid t=r+\vartheta \gamma h}\right|^{q} d y\right)^{1 / q} d \gamma d \vartheta
$$

Therefore $\|\widetilde{Z}\|_{1, q}+\|\mathfrak{D}(Z)\|_{1, q} \leq \mathfrak{C}|h|$ by the second estimate in Corollary 3.3 with $K=R^{\prime}$. This proves differentiability of $\mathfrak{G}(x, \cdot, r)$ with respect to the norm of $W^{1, q}\left(B_{R_{1}}\right)^{3 \times 3}$, as well as equation (3.14). Continuity of $\partial_{r} \mathfrak{G}(x, \cdot, r)$ with respect to the same norm follows by a similar argument.

In the preceding lemma, we considered the derivative of $\mathfrak{G}(x, \cdot, r)$ with respect to $r$. Next we deal with derivatives with respect to $x_{l}$, for $1 \leq l \leq 3$.

Lemma 3.13 Let $q \in(1, \infty), \sigma \in\{0,1\}$. For $x \in B_{R_{0}}^{c}, r \in[0, \infty)$, define $L(x, r)$ : $B_{R_{1}} \mapsto \mathbb{R}^{3 \times 3}$ by $L(x, r)(y):=\partial_{r}^{\sigma} \mathfrak{G}(x, y, r)\left(y \in B_{R_{1}}\right)$; see Lemma 3.12 for the definition of $\partial_{r}^{\sigma} \mathfrak{G}(x, y, r)$ if $\sigma=1$. Then $L(x, r) \in C_{0}^{\infty}\left(B_{R_{1}}\right)^{3 \times 3} \cap W^{1, q}\left(B_{R_{1}}\right)^{3 \times 3}$ for $x, r$ as before, and the mapping $L(\cdot, r)$ considered as an operator from $B_{R_{0}}^{c}$ into $W^{1, q}\left(B_{R_{1}}\right)^{3 \times 3}$ is partially differentiable on $\overline{B_{R_{0}}}$. Thus we may define a mapping $D_{m} L:{\overline{B_{R_{0}}}}^{c} \times[0, \infty) \mapsto W^{1, q}\left(B_{R_{1}}\right)^{3 \times 3}$ by the condition $\left\|\left(L\left(x+h e_{m}, r\right)-L(x, r)\right) / h-D_{m} L(x, r)\right\|_{1, q} \rightarrow 0(h \rightarrow 0)$, for $m \in$ $\{1,2,3\}, x \in \overline{B_{R_{0}}}, r \in[0, \infty)$. The preceding transition to the limit is uniform with respect to $r \in[0, \infty)$. Instead of $D_{m} L(x, r)(y)$, we write $\partial x_{m} \partial_{r}^{\sigma} \mathfrak{G}(x, y, r)$. Then

$$
\left.\partial x_{m} \partial_{r}^{\sigma} \mathfrak{G}_{j k}(x, y, r)=\varphi_{0}(y) \partial x_{m} \partial_{r}^{\sigma} \Lambda_{j k}(x-y, r)\right)-\mathfrak{D}\left[\partial x_{m} \partial_{r}^{\sigma} M_{\varphi_{0}, j}(x, \cdot, r)\right]_{k}(y)
$$

for $x \in{\overline{B_{R_{0}}}}^{c}, y \in B_{R_{1}}, r \in[0, \infty), j, k, m \in\{1,2,3\}$, where the operator $\mathfrak{D}:=\mathfrak{D}_{R_{1}, S_{0}}$ was introduced in Theorem 2.4.

Let $l \in\{1,2,3\}$ and define $\widetilde{L}(x, r): B_{R_{1}} \mapsto \mathbb{R}^{3 \times 3}$ by $\widetilde{L}(x, r)(y):=\partial y_{l} \mathfrak{G}(x, y, r)$ for $x \in$ $B_{R_{0}}^{c}, r \in[0, \infty), y \in B_{R_{1}}$. Then $\widetilde{L}(x, r) \in C_{0}^{\infty}\left(B_{R_{1}}\right)^{3 \times 3} \cap L^{q}\left(B_{R_{1}}\right)^{3 \times 3}$ for $x, r$ as before, 
and $\widetilde{L}(\cdot, r)$ considered as an operator from $B_{R_{0}}^{c}$ into $L^{q}\left(B_{R_{1}}\right)^{3 \times 3}$ is partially differentiable on ${\overline{B_{R_{0}}}}^{c}$. Thus we may define an operator $D_{m} \widetilde{L}:{\overline{B_{R_{0}}}}^{c} \times[0, \infty) \mapsto L^{q}\left(B_{R_{1}}\right)^{3 \times 3}$ by the condition $\left\|\left(\widetilde{L}\left(x+h e_{m}, r\right)-\widetilde{L}(x, r)\right) / h-D_{m} \widetilde{L}(x, r)\right\|_{q} \rightarrow 0(h \rightarrow 0)(m \in\{1,2,3\}, x \in$ \left.${\overline{B_{R_{0}}}}^{c}, r \in[0, \infty)\right)$. The preceding transition to the limit is uniform with respect to $r \in$ $[0, \infty)$. Instead of $D_{m} \widetilde{L}(x, r)(y)$, we write $\partial x_{m} \partial y_{l} \mathfrak{G}(x, y, r)$ so that

$$
\begin{aligned}
& \partial x_{m} \partial y_{l} \mathfrak{G}_{j k}(x, y, r) \\
& =\partial y_{l}\left(\varphi_{0}(y) \partial x_{m} \Lambda_{j k}(x-y, r)\right)-\partial y_{l}\left(\mathfrak{D}\left[\partial x_{m} M_{\varphi_{0}, j}(x, \cdot, r)\right]_{k}\right)(y)
\end{aligned}
$$

for $x \in{\overline{B_{R_{0}}}}^{c}, r \in[0, \infty), y \in B_{R_{1}}, j, k, m \in\{1,2,3\}$.

Proof: The relations $L(x, r), \widetilde{L}(x, r) \in C_{0}^{\infty}\left(B_{R_{1}}\right)^{3 \times 3}$ for $x \in B_{R_{0}}^{c}, r \in[0, \infty)$ hold due to Lemma 3.11 ( $L$ with $\sigma=0, \widetilde{L}$ ), (3.14), Lemma 3.3, 3.10, Theorem 2.4 and the choice of $\varphi_{0}(L$ with $\sigma=1)$.

As concerns differentiability of $L(x, r)$ with respect to $x$, let $j, k, m \in\{1,2,3\}, \beta \in \mathbb{N}_{0}^{3}$ with $|\beta| \leq 1$, and put $L_{j k}^{(1)}(x, r)(y):=-\partial_{y}^{\beta} \mathfrak{D}\left(\partial_{r}^{\sigma} M_{\varphi_{0}, j}(x, \cdot, r)\right)_{k}(y), L_{j k}^{(2)}(x, r)(y):=$ $\partial_{y}^{\beta} \varphi_{0}(y) \partial_{r}^{\sigma} \Lambda(x-y, r)$ and $L_{j k}^{(3)}(x, r)(y):=\varphi_{0}(y) \partial_{y}^{\beta} \partial_{r}^{\sigma} \Lambda(x-y, r)$ for $x \in{\overline{B_{R_{0}}}}^{c}, \quad r \in$ $[0, \infty), y \in B_{R_{1}}$. If $x \in \overline{B_{R_{0}}} c h \in \mathbb{R}$ with $|h|<\operatorname{dist}\left(x, B_{R_{0}}\right)$ and $y \in B_{R_{1}}$, we have $\left|x+h e_{m}-y\right| \geq\left(R_{0}-S_{0}\right) / 2$. Thus, by a similar reasoning as in the proof of Lemma 3.12, we get $\left[\int_{B_{R_{1}}}\left|\left(L_{j k}^{(\mu)}\left(x+h e_{m}, r\right)(y)-L_{j k}^{(\mu)}(x, r)(y)\right) / h-D_{m} L_{j k}^{(\mu)}(x, r)(y)\right|^{q} d y\right]^{1 / q} \leq \mathfrak{C}|h|$ for $x \in \overline{B_{R_{0}}}, r \in[0, \infty), \mu \in\{1,2,3\}, h \in \mathbb{R} \backslash\{0\}$ with $|h| \leq \operatorname{dist}\left(x, B_{R_{0}}\right)$, with an obvious definition of $D_{m} L_{j k}^{(\mu)}(x, r)(y)$. This implies the differentiability properties of $L(\cdot r)$ as stated in the lemma, as well as equation (3.15). Differentiability of $\widetilde{L}(\cdot, r)$ and equation (3.16) follow from corresponding results on $L(\cdot, r)$.

We fix some function $\zeta \in C^{\infty}(\mathbb{R})$ with $\zeta|(-\infty, 1]=0, \zeta|[2, \infty)=1,0 \leq \zeta \leq 1$ and $\zeta^{\prime} \geq 0$. For $\epsilon \in(0, \infty), r \in \mathbb{R}$, define $\zeta_{\epsilon}(r):=\zeta(r / \epsilon)$. Then $\zeta_{\epsilon} \in C^{\infty}(\mathbb{R}), \zeta \mid(-\infty, \epsilon]=$ $0, \zeta \mid[2 \epsilon, \infty)=1,0 \leq \zeta_{\epsilon} \leq 1$ and $\zeta_{\epsilon}^{\prime} \geq 0$.

Lemma 3.14 Let $q \in(1, \infty), T_{0} \in(0, \infty], v \in C^{0}\left(\left[0, T_{0}\right), L^{q}\left(B_{R_{1}}\right)^{3}\right), t \in\left(0, T_{0}\right), x \in$ $B_{R_{0}}^{c}$. Then

$$
\int_{0}^{t} \int_{B_{R_{1}}} \mathfrak{G}(x, y, t-s) \cdot \zeta_{\epsilon}^{\prime}(s) v(y, s) d y d s-\int_{B_{R_{1}}} \mathfrak{G}(x, y, t) \cdot v(y, 0) d y \rightarrow 0(\epsilon \downarrow 0) .
$$

Proof: For $\epsilon \in(0, \infty)$, let $A_{\epsilon}$ denote the difference of two integrals considered in (3.17). Then $A_{\epsilon}=A_{\epsilon}^{(1)}+A_{\epsilon}^{(2)}$, with

$$
\begin{aligned}
& A_{\epsilon}^{(1)}:=\int_{0}^{t} \int_{B_{R_{1}}}(\mathfrak{G}(x, y, t-s)-\mathfrak{G}(x, y, t)) \cdot \zeta_{\epsilon}^{\prime}(s) v(y, s) d y d s \\
& A_{\epsilon}^{(2)}:=\int_{0}^{t} \int_{B_{R_{1}}} \mathfrak{G}(x, y, t) \cdot \zeta_{\epsilon}^{\prime}(s) v(y, s) d y d s-\int_{B_{R_{1}}} \mathfrak{G}(x, y, t) \cdot v(y, 0) d y
\end{aligned}
$$

for $\epsilon \in(0, \infty)$. By Lemma 3.12, the mapping $r \mapsto \mathfrak{G}(x, \cdot, r)(r \in[0, \infty))$ belongs in particular to $C^{0}\left([0, \infty), L^{q^{\prime}}\left(B_{R_{1}}\right)^{3 \times 3}\right)$, so $\sigma(\epsilon):=\sup _{s \in[\epsilon, 2 \epsilon]}\|G(x, \cdot, t-s)-G(x, \cdot, t)\|_{q^{\prime}} \rightarrow 0$ 
for $\epsilon \downarrow 0$. On the other hand, $\operatorname{supp}\left(\zeta^{\prime}\right) \subset[\epsilon, 2 \epsilon]$ for $\epsilon>0$, so for $\epsilon \in(0, t / 4]$, we find that $\left|A_{\epsilon}^{(1)}\right| \leq \int_{\epsilon}^{2 \epsilon} \zeta_{\epsilon}^{\prime}(s) d s \sigma(\epsilon)\left\|v \mid B_{R_{1}} \times[0,2 \epsilon]\right\|_{q, \infty ; 2 \epsilon}$, where we used that $\zeta_{\epsilon} \geq 0$. Since $\int_{\epsilon}^{2 \epsilon} \zeta_{\epsilon}^{\prime}(s) d s=1$, we may conclude that $A_{\epsilon}^{(1)} \rightarrow 0(\epsilon \downarrow 0)$. By Lemma 3.12, we know that in particular $\mathfrak{G}(x, \cdot, t) \in L^{q^{\prime}}\left(B_{R_{1}}\right)^{3 \times 3}$. This fact and the relations $\zeta_{\epsilon}^{\prime} \geq 0, \int_{\epsilon}^{2 \epsilon} \zeta_{\epsilon}^{\prime}(s) d s=1$ imply

$$
\left|A_{\epsilon}^{(2)}\right| \leq\left|\int_{\epsilon}^{2 \epsilon} \zeta_{\epsilon}^{\prime}(s) \int_{B_{R_{1}}} \mathfrak{G}(x, y, t)(v(y, s)-v(y, 0)) d y d s\right| \leq \mathfrak{C} \sup _{s \in[\epsilon, 2 \epsilon]}\|v(s)-v(0)\|_{q}
$$

for $\epsilon>0$ with $2 \epsilon<T_{0}$. Since $v \in C^{0}\left(\left[0, T_{0}\right), L^{q}\left(B_{R_{1}}\right)^{3}\right)$, the term $\sup _{s \in[\epsilon, 2 \epsilon]}\|v(s)-v(0)\|_{q}$ tends to zero for $\epsilon \downarrow 0$. Thus we get $A_{\epsilon}^{(2)} \rightarrow 0(\epsilon \downarrow 0)$. Altogether we see that $A_{\epsilon} \rightarrow 0(\epsilon \downarrow 0)$. This proves the lemma.

Lemma 3.15 Let $q \in(1, \infty), T_{0} \in(0, \infty], u \in C^{0}\left(\left[0, T_{0}\right), L^{q}\left(\mathbb{R}^{3}\right)^{3}\right), t \in\left(0, T_{0}\right), x \in$ $\mathbb{R}^{3}$. Put $\varrho_{\epsilon}(s):=\zeta_{\epsilon}^{\prime}(s) u(s)$ for $s \in\left(0, T_{0}\right), \epsilon \in(0, \infty)$. Then $\mathfrak{R}^{(\tau)}\left(\varrho_{\epsilon}\right)(x, t)$ converges to $\mathfrak{I}^{(\tau)}(u(0))(x, t)$ for $\epsilon \downarrow 0$.

Proof: For $\epsilon \in(0, \infty)$, we have $\mathfrak{R}^{(\tau)}\left(\varrho_{\epsilon}\right)(x, t)-\mathfrak{I}^{(\tau)}(u(0))(x, t)=A_{\epsilon}^{(1)}+A_{\epsilon}^{(2)}$, with

$$
\begin{aligned}
& A_{\epsilon}^{(1)}:=\int_{0}^{t} \int_{\mathbb{R}^{3}}(\Lambda(x-y, t-s)-\Lambda(x-y, t)) \cdot \zeta_{\epsilon}^{\prime}(s) u(y, s) d y d s, \\
& A_{\epsilon}^{(2)}:=\int_{0}^{t} \int_{\mathbb{R}^{3}} \Lambda(x-y, t) \cdot \zeta_{\epsilon}^{\prime}(s) u(y, s) d y d s-\mathfrak{I}^{(\tau)}(u(0))(x, t) .
\end{aligned}
$$

Recalling that $\operatorname{supp}\left(\zeta_{\epsilon}^{\prime}\right) \subset[\epsilon, 2 \epsilon], \zeta_{\epsilon}^{\prime} \geq 0$, we get for $\epsilon \in(0, t / 4]$ that

$$
\left|A_{\epsilon}^{(1)}\right| \leq \int_{\epsilon}^{2 \epsilon} \zeta_{\epsilon}^{\prime}(s) \mathfrak{B}(s)^{1 / q^{\prime}}\|u(s)\|_{q} d s,
$$

with $\mathfrak{B}(s):=\int_{\mathbb{R}^{3}}|\Lambda(x-y, t-s)-\Lambda(x-y, t)|^{q \prime} d y$ for $s \in(0, t)$. On the other hand, $t-\vartheta s \geq t / 2$ for $\epsilon \in(0, t / 4], s \in[\epsilon, 2 \epsilon], \vartheta \in[0,1]$. Since $\Lambda$ is $C^{\infty}$ on $\mathbb{R}^{3} \times(0, \infty)$ (Lemma $3.3)$, we obtain $\mathfrak{B}(s)=s \int_{\mathbb{R}^{3}}\left|\int_{0}^{1} \partial_{t} \Lambda(x-y, t-\vartheta s)\right|^{q^{\prime}} d y$ if $\epsilon \in(0, t / 4]$ and $s \in[\epsilon, 2 \epsilon]$. Changing the order of integration by using Theorem 2.1, and applying Corollary 3.4, we thus see that $\mathfrak{B}(s)^{1 / q^{\prime}}$ is bounded by $\mathfrak{C} s \sum_{\mu=1}^{2} \int_{0}^{1}(t-\vartheta s)^{-(3+\mu) / 2+3 /\left(2 q^{\prime}\right)} d \vartheta$, and hence by $\mathfrak{C} s \sum_{\mu=1}^{2} t^{-(3+\mu) / 2+3 /\left(2 q^{\prime}\right)}$, for $\epsilon$ and $s$ as before. Now we may conclude from (3.18) that

$$
\left|A_{\epsilon}^{(1)}\right| \leq \mathfrak{C} \int_{\epsilon}^{2 \epsilon} s \zeta_{\epsilon}^{\prime}(s) d s \sum_{\mu=1}^{2} t^{-(3+\mu) / 2+3 /\left(2 q^{\prime}\right)} \max \left\{\|u\|_{q}: s \in[\epsilon, 2 \epsilon]\right\}
$$

for $\epsilon \in(0, t / 4]$. But $\zeta_{\epsilon}^{\prime} \geq 0, \int_{\epsilon}^{2 \epsilon} \zeta_{\epsilon}^{\prime}(s) d s=1$, so $\int_{\epsilon}^{2 \epsilon} s \zeta_{\epsilon}^{\prime}(s) d s \leq 2 \epsilon$, hence $A_{\epsilon}^{(1)} \rightarrow 0(\epsilon \downarrow 0)$. Again using the equation $\int_{\epsilon}^{2 \epsilon} \zeta_{\epsilon}^{\prime}(s) d s=1$ and the relations $\zeta_{\epsilon}^{\prime} \geq 0, \operatorname{supp}\left(\zeta_{\epsilon}^{\prime}\right) \subset[\epsilon, 2 \epsilon]$, we get $\left|A_{\epsilon}^{(2)}\right| \leq \mathfrak{C} \int_{\epsilon}^{2 \epsilon} \zeta_{\epsilon}^{\prime}(s) d s \int_{\mathbb{R}^{3}}|\Lambda(x-y, t) \cdot(u(y, s)-u(y, 0))| d y d s$ for $\epsilon \in(0, t / 4]$. Corollary 3.4 yields in particular that the function $y \mapsto \Lambda(x-y, t)\left(y \in \mathbb{R}^{3}\right)$ belongs to $L^{q^{\prime}}\left(\mathbb{R}^{3}\right)^{3 \times 3}$. Thus it follows as in a similar situation in the proof of Lemma 3.14 that $A_{\epsilon}^{(2)} \rightarrow 0(\epsilon \downarrow 0)$. This completes the proof of Lemma 3.15. 


\section{Some decay estimates}

In this section, we derive decay estimates of the potential functions introduced in the preceding one. We begin with a technical tool which is in fact the key result which will allow us to improve the decay rates exhibited in [10] and [13].

Theorem 4.1 Abbreviate $K(z, r):=\left|z-\tau r e_{1}\right|^{2}+r$ for $z \in \mathbb{R}^{3}, r \in(0, \infty)$. Let $n \in$ $(2, \infty), R \in(0, \infty)$. Then

$$
\int_{0}^{\infty} K(z, r)^{-n / 2} d r \leq C(n, R, \tau)(|z| \nu(z))^{(-n+1) / 2} \quad \text { for } z \in B_{R}^{c} .
$$

In addition, let $\widetilde{R} \in(0, \infty)$ with $R<\widetilde{R}, p, q \in[1, \infty)$. Then

$$
\begin{aligned}
& \left(\int_{0}^{t}\left(\int_{B_{R}} K(x-y, t-s)^{-n q / 2} d y\right)^{p / q} d s\right)^{1 / q} \leq \mathfrak{C}(|x| \nu(x))^{-n / 2+1 /(2 p)}, \\
& \left(\int_{B_{R}} K(x-y, t)^{-n q / 2} d y\right)^{1 / q} \leq \mathfrak{C}(|x| \nu(x))^{-n / 2}
\end{aligned}
$$

for $t \in(0, \infty), x \in B_{\widetilde{R}}^{c}$. In addition, let $a \in(1, \infty]$. Then

$$
\int_{0}^{t} \int_{B_{R}} K(x-y, t-s)^{-n / 2}|u(y, s)| d y d s \leq \mathfrak{C}(|x| \nu(x))^{-n / 2+1 /\left(2 a^{\prime}\right)}\|u\|_{1, a ; t}
$$

for $t, x$ as before and for $u \in L^{a}\left(0, t, L^{1}\left(B_{R}\right)\right)$.

We remark that inequality (4.1) is a special case of [21, Theorem 2.19]

Proof of Theorem 4.1: Let $S, r \in(0, \infty), y \in B_{S}, x \in \mathbb{R}^{3}$. Then $K(x-y, r)=$ $(|x-y|-\tau r)^{2}+\left[1+2 \tau\left(|x-y|-(x-y)_{1}\right)\right] r$. But $|x-y|-(x-y)_{1} \geq 0$, so we obtain that $K(x-y, r)^{1 / 2} \geq 2^{-1 / 2}\left(|| x-y|-\tau r|+\left[1+2 \tau\left(|x-y|-(x-y)_{1}\right)\right]^{1 / 2} r^{1 / 2}\right)$. Put $C_{1}:=\min \left\{(2 S+1)^{-1}, 2 \tau\right\}$. Since $|y| \leq S$, we get $1+2 \tau\left(|x-y|-(x-y)_{1}\right) \geq$ $C_{1}\left(2 S+1+|x-y|-(x-y)_{1}\right) \geq C_{1}\left(2 S+1+|x|-x_{1}-2|y|\right) \geq C_{1}\left(1+|x|-x_{1}\right)=C_{1} \nu(x)$, so

$$
K(x-y, r)^{1 / 2} \geq C(S, \tau)\left(|| x-y|-\tau r|+[\nu(x) r]^{1 / 2}\right) .
$$

Now suppose that $x \in B_{2 S}^{c}$ so that $|x|-S \geq|x| / 2$. If $r \in(0,|x| /(4 \tau)]$, we then obtain the estimate $|x-y|-\tau r \geq|x|-S-\tau r \geq|x| / 2-\tau r \geq|x| / 4>0$, and thus $K(x-y, r)^{1 / 2} \geq C(S, \tau)(|x| / 2-\tau r)>0$. Next consider the case $r \in[|x| /(4 \tau),(|x|-S) / \tau]$. Using that $|y| \leq S$, we find $|x-y|-\tau r \geq|x|-S-\tau r \geq 0$ and $r^{1 / 2} \geq|x|^{1 / 2}\left(2 \tau^{1 / 2}\right)^{-1}$, so from (4.5), $K(x-y, r)^{1 / 2} \geq C(S, \tau)\left[|x|-S-\tau r+(\nu(x)|x|)^{1 / 2}\right]>0$. Now turn to the case that $r \in[(|x|-S) / \tau,(|x|+S) / \tau]$. Since $|x|-S \geq|x| / 2$, as remarked above, we then have $r \geq(|x|-S) / \tau \geq|x| /(2 \tau)$. Thus inequality (4.5) yields $K(x-y, r)^{1 / 2} \geq$ $C(S, \tau)(\nu(x)|x|))^{1 / 2}>0$. Finally, if $r \geq(|x|+S) / \tau$, we have on the one hand $r \geq|x| / \tau$, and on the other one, $\tau r-|x-y| \geq \tau r-(|x|+S) \geq 0$, so by $(4.5), K(x-y, r)^{1 / 2} \geq$ $C(S, \tau)\left[\tau r-(|x|+S)+(\nu(x)|x|)^{1 / 2}\right]>0$.

Define $F_{S}(x, r)$ by $F_{S}(x, r):=|x| / 2-\tau r$ if $r<|x| /(4 \tau), F_{S}(x, r):=|x|-S-\tau r+$ $(\nu(x)|x|)^{1 / 2}$ if $|x| /(4 \tau) \leq r \leq(|x|-S) / \tau, F_{S}(x, r):=(\nu(x)|x|)^{1 / 2}$ if $(|x|-S) / \tau \leq r \leq$ 
$(|x|+S) / \tau$, and $F_{S}(x, r):=\tau r-(|x|+S)+(\nu(x)|x|)^{1 / 2}$ in the case $(|x|+S) / \tau<r$. Then we have shown that

$$
K(x-y, r)^{1 / 2} \geq C(S, \tau) F_{S}(x, r)>0 \quad \text { for } S \in(0, \infty), y \in B_{S}, x \in B_{2 S}^{c}, r>0 .
$$

For such $S, x$ and $y$, and for $\gamma>1$, we get

$$
\begin{aligned}
& \int_{0}^{|x| /(4 \tau)} F_{S}(x, r)^{-\gamma} d r=\tau^{-1} \int_{0}^{|x| / 4}(|x| / 2-t)^{-\gamma} d t \leq C(\gamma, \tau)|x|^{-\gamma+1}, \\
& \int_{|x| /(4 \tau)}^{(|x|-S) / \tau} F_{S}(x, r)^{-\gamma} d r+\int_{(|x|+S) / \tau}^{\infty} F_{S}(x, r)^{-\gamma} d r \leq C(\gamma, \tau)(|x| \nu(x))^{(-\gamma+1) / 2},
\end{aligned}
$$

and $\int_{(|x|-S) / \tau}^{(|x|+S) / \tau} F_{S}(x, r)^{-\gamma} d r=(|x| \nu(x))^{-\gamma / 2} 2 S / \tau$. Since $|x| \geq C(S) \nu(x)$ for $x \in B_{2 S}^{c}$, we may conclude that

$$
\int_{0}^{\infty} F_{S}(x, r)^{-\gamma} d r \leq C(\gamma, \tau, S)(|x| \nu(x))^{(-\gamma+1) / 2} \quad \text { for } S, \gamma \in(0, \infty),
$$

and for $x \in B_{2 S}^{c}, y \in B_{S}$. Taking $S=R / 2, y=0, \gamma=n$ in (4.7) and using (4.6), we obtain (4.1). Due to (4.6), the left-hand side in (4.2) is bounded by the term $C(R, \tau)\left(\int_{0}^{t} F_{R}(x, t-s)^{-p n} d s\left[\int_{B_{R}} d y\right]^{p / q}\right)^{1 / p}$ for $t \in(0, \infty), x \in B_{2 R}^{c}$, so (4.2) follows with (4.7) for such $t$ and $x$. We still have to show (4.2) in the case $2 R>\widetilde{R}, x \in B_{2 R} \backslash B_{\widetilde{R}}$. Taking account only of the assumption $\widetilde{R}>R$, we get by Corollary 3.1 that

$$
K(x-y, r) \geq C(\widetilde{R}, R, \tau)(|x| \nu(x)+r) \quad \text { for } x \in B_{\widetilde{R}}^{c}, y \in B_{R}, r \in(0, \infty),
$$

so the left-hand side of (4.2) is bounded by $\mathfrak{C}\left[\int_{0}^{t}(|x| \nu(x)+t-s)^{-p n / 2} d s\left(\int_{B_{R}} d y\right)^{p / q}\right]^{1 / q}$ for $t \in(0, \infty), x \in B_{\widetilde{R}}^{c}$, and hence by $\mathfrak{C}(|x| \nu(x))^{-n / 2+1 / p}$. But if $2 R>\widetilde{R}$ and $x \in$ $B_{2 R} \backslash B_{\widetilde{R}}$, we further get $1 \leq 2 R /|x|$ and $|x| \geq C(\widetilde{R}) \nu(x)$, so

$$
(|x| \nu(x))^{-n / 2+1 / p} \leq(2 R)^{1 / p}(|x| \nu(x))^{-n / 2+1 / p}|x|^{-1 / p} \leq \mathfrak{C}(|x| \nu(x))^{-n / 2+1 /(2 p)} .
$$

Therefore the estimate in (4.2) holds again. Inequality (4.3) is an immediate consequence or (4.8). In order to prove (4.4), let $\mathfrak{A}$ denote the left-hand side of (4.4) Then by (4.6),

$$
\mathfrak{A} \leq \mathfrak{C} \int_{0}^{t} \int_{B_{R}} F_{R}(x, t-s)^{-n}|u(y, s)| d y d s \leq \mathfrak{C}\left(\int_{0}^{t} F_{R}(x, t-s)^{-n a^{\prime}} d s\right)^{1 / a^{\prime}}\|u\|_{1, a ; t}(4
$$

for $t \in(0, \infty), x \in B_{2 R}^{c}$. Now (4.4) follows from (4.7). If $2 R>\widetilde{R}$ and $x \in B_{2 R} \backslash B_{\widetilde{R}}$, we proceed as in (4.10), replacing (4.6) by (4.8). After integration, we use (4.9) with $a^{\prime}$ in the place of $p$.

In the ensuing corollary, we apply the preceding theorem in order to estimate convolutions of the Oseen fundamental solution $\Lambda$ in the case that the integration with respect to the space variables only extends over a bounded domain. 
Corollary 4.1 Let $R, \widetilde{R} \in(0, \infty)$ with $R<\widetilde{R}, p, q \in[1, \infty]$. Then

$$
\int_{0}^{t} \int_{B_{R}}\left|\partial_{x}^{\alpha} \partial_{y}^{\beta} \partial_{t}^{\sigma} \Lambda(x-y, t-s) u(y, s)\right| d y d s \leq \mathfrak{C}(|x| \nu(x))^{-(3+|\alpha|+|\beta|+\sigma) / 2+1 /\left(2 p^{\prime}\right)}\|u\|_{q, p ; t}
$$

for $u \in L^{p}\left(0, t, L^{q}\left(B_{R}\right)^{3}\right), \alpha, \beta \in \mathbb{N}_{0}^{3}$ with $|\alpha| \leq 2,|\beta| \leq 1, \sigma \in\{0,1\}, t \in(0, \infty), x \in$ $B_{\widetilde{R}}^{c}$.

Proof: Let the left-hand side of the preceding inequality be denoted by $\mathfrak{A}$. As in Theorem 4.1, abbreviate $K(z, r):=\left|z-\tau r e_{1}\right|^{2}+r$ for $z \in \mathbb{R}^{3}, r \in(0, \infty)$. By the first inequality in Corollary 3.3, we have for $u, \alpha, \beta, \sigma, t, x$ as in the corollary,

$$
\mathfrak{A} \leq C(\tau) \sum_{\mu=1}^{2} \int_{0}^{t} \int_{B_{R}} K(x-y, t-s)^{-(3+|\alpha|+|\beta|+\mu \sigma) / 2}|u(y, s)| d y d s .
$$

Suppose that $p, q \in(1, \infty]$. Then from the preceding estimate and (4.2),

$$
\begin{aligned}
& \mathfrak{A} \leq \mathfrak{C} \sum_{\mu=1}^{2}\left(\int_{0}^{t}\left(\int_{B_{R}} K(x-y, t-s)^{-(3+|\alpha|+|\beta|+\mu \sigma) q^{\prime} / 2} d y\right)^{p^{\prime} / q^{\prime}} d s\right)^{1 / p^{\prime}}\|u\|_{q, p ; t} \\
& \leq \mathfrak{C} \sum_{\mu=1}^{2}(|x| \nu(x))^{-(3+|\alpha|+|\beta|+\mu \sigma) / 2+1 /\left(2 p^{\prime}\right)}\|u\|_{q, p ; t}
\end{aligned}
$$

for $u, \alpha, \beta, \sigma, t, x$ as before. But $(|x| \nu(x))^{-\sigma / 2} \leq C(\widetilde{R})$ for $x \in B_{\widetilde{R}}^{c}$, so the corollary is proved in the case $p, q \in(1, \infty]$. Now consider the case $q \in(1, \infty], p=1$. Then we again start with (4.11), to obtain

$$
\mathfrak{A} \leq \mathfrak{C} \sum_{\mu=1}^{2} \int_{0}^{t}\left(\int_{B_{R}} K(x-y, t-s)^{-(3+|\alpha|+|\beta|+\mu \sigma) q^{\prime} / 2} d y\right)^{1 / q^{\prime}}\|u(s)\|_{q} d s
$$

for $u, \alpha, \beta, \sigma, t, x$ as in the corollary, which now follows with (4.3). The case $q=1, p \in$ $(1, \infty]$ is handled by referring to (4.11) and (4.4). Finally, if $p=q=1$, we use the estimate $K(x-y, t-s) \geq C(R, \widetilde{R}, \tau)(|x| \nu(x)+t-r) \geq C(R, \widetilde{R}, \tau)|x| \nu(x)$ for $t \in(0, \infty), x \in$ $B_{\widetilde{R}}^{c}, y \in B_{R}, r \in(0, t)$ (Corollary 3.1), which together with (4.11) immediately implies the corollary.

Recall the numbers $S_{0}, R_{0}, R_{1}$ and the function $\varphi_{0}$ fixed at the beginning of Section 2 . In the rest of this section, we abbreviate $\mathfrak{G}:=\mathfrak{G}_{R_{0}, S_{0}, \varphi_{0}}$, where $\mathfrak{G}_{R_{0}, S_{0}, \varphi_{0}}$ was defined in (3.13). The ensuing theorem is the main result of this section and the key element which will allow us in the next section to exploit our representation formula of solutions to (1.1), improving decay rates compared to [10] and [13] while at the same time dropping boundary conditions.

Theorem 4.2 Let $q \in(1, \infty), p \in[1, \infty]$. Then

$$
\int_{B_{R_{1}}}\left|\partial_{x}^{\alpha} \partial_{t}^{\sigma} \partial_{y}^{\beta} \mathfrak{G}(x, y, t) \cdot V(y)\right| d y \leq \mathfrak{C}(|x| \nu(x))^{-(3+|\alpha|+\sigma) / 2}\|V\|_{q}
$$


for $V \in L^{q}\left(B_{R_{1}}\right)^{3}, t \in(0, \infty), x \in B_{R_{0}}^{c}, \alpha, \beta \in \mathbb{N}_{0}^{3}, \sigma \in\{0,1\}$ with $|\alpha| \leq 1,|\beta|+\sigma \leq 1$,

$\int_{0}^{t} \int_{B_{R_{1}}}\left|\partial_{x}^{\alpha} \partial_{t}^{\sigma} \partial_{y}^{\beta} \mathfrak{G}(x, y, t-s) \cdot v(y, s)\right| d y d s \leq \mathfrak{C}(|x| \nu(x))^{-(3+|\alpha|+\sigma) / 2+1 /\left(2 p^{\prime}\right)}\|v\|_{q, p ; t}(4$

for t, $x, \alpha, \beta, \sigma$ as in (4.12), and $v \in L^{p}\left(0, t, L^{q}\left(B_{R_{1}}\right)^{3}\right)$.

Proof: Take $V, v, t, x, \alpha, \beta, \sigma$ as in the theorem. Moreover let $j, k \in\{1,2,3\}, s \in(0, t)$ Suppose that $r, W$ are either given by $r:=t, W:=V$ or by $r:=t-s$ and $W:=v(s)$. Then by (3.14), (3.15) and (3.16),

$$
\mathfrak{A}(r):=\int_{B_{R_{1}}}\left|\partial_{x}^{\alpha} \partial_{r}^{\sigma} \partial_{y}^{\beta} \mathfrak{G}_{j k}(x, y, r) W_{k}(y)\right| d y \leq \sum_{m=1}^{3} \mathfrak{A}^{(m)}(r),
$$

with $\mathfrak{A}^{(1)}(r):=\int_{B_{R_{1}}}\left|\delta_{1,|\beta|} \partial^{\beta} \varphi_{0}(y) \partial_{x}^{\alpha} \partial_{r}^{\sigma} \Lambda_{j k}(x-y, r) W_{k}(y)\right| d y$, and the term $\mathfrak{A}^{(3)}(r)$ being an abbreviation for the integral $\int_{B_{R_{1}}}\left|\partial_{y}^{\beta} \mathfrak{D}\left(\partial_{x}^{\alpha} \partial_{r}^{\sigma} M_{\varphi_{0}, j}(x, r)\right)_{k}(y) W_{k}(y)\right| d y$. The term $\mathfrak{A}^{(2)}(r)$ is defined as $\mathfrak{A}^{(1)}(r)$, but with the function $\delta_{1,|\beta|} \partial^{\beta} \varphi_{0}(y) \partial_{x}^{\alpha} \partial_{r}^{\sigma} \Lambda_{j k}(x-y, r)$ replaced by $\varphi_{0}(y) \partial_{x}^{\alpha} \partial_{r}^{\sigma} \partial_{y}^{\beta} \Lambda_{j k}(x-y, r)$. The function $M_{\varphi_{0}}$ was defined in (3.12), and the operator $\mathfrak{D}:=\mathfrak{D}_{R_{1}, S_{0}}$ in Theorem 2.4. Set $K(z, r):=\left|z-\tau r e_{1}\right|^{2}+r$ for $z \in \mathbb{R}^{3}$. Then by the first inequality in Corollary 3.3 and Hölder's inequality, we have

$$
\mathfrak{A}^{(1)}(r) \leq C \sum_{\mu=1}^{2}\left(\int_{B_{R_{1}}} K(x-y, r)^{-(3+|\alpha|+\mu \sigma) q^{\prime} / 2} d y\right)^{1 / q^{\prime}}\|W\|_{q} .
$$

The same estimate is valid for $\mathfrak{A}^{(2)}(r)$, except that the exponent $-(3+|\alpha|+\mu \sigma) q^{\prime} / 2$ has to be replaced by $-(3+|\alpha|+|\beta|+\mu \sigma) q^{\prime} / 2$. Put $A:=A_{R_{1}, S_{0}}$. Since $\partial_{x}^{\alpha} \partial_{r}^{\sigma} M_{\varphi_{0}}(x, \cdot, r) \in$ $C_{0}^{\infty}(A)^{3}$ with mean value zero on $A$ (Lemma 3.10), Hölder's inequality and Theorem 2.4 yield $\mathfrak{A}^{(3)}(r) \leq C\left(R_{0}, S_{0}, q\right)\left(\int_{A}\left|\partial_{x}^{\alpha} \partial_{r}^{\sigma} M_{\varphi_{0}}(x, y, r)\right|^{q^{\prime}} d y\right)^{1 / q^{\prime}}\|W\|_{q}$. But $\left|\partial_{x}^{\alpha} \partial_{r}^{\sigma} M_{\varphi_{0}}(x, y, r)\right|$ is bounded by $C\left|\nabla \varphi_{0}\right|_{\infty}\left|\partial_{x}^{\alpha} \partial_{r}^{\sigma} \Lambda(x-y, r)\right|$ for $y \in A$, and so in view of the first inequality in Corollary 3.1 by $C(\tau)\left|\nabla \varphi_{0}\right|_{\infty} \sum_{\mu=1}^{2} K(x-y, r)^{-(3+|\alpha|+\mu \sigma) / 2}$. Therefore

$$
\mathfrak{A}^{(3)}(r) \leq C\left(R_{0}, S_{0}, q, \tau\right)\left|\nabla \varphi_{0}\right|_{\infty}\left(\int_{A} \sum_{\mu=1}^{2} K(x-y, r)^{-(3+|\alpha|+\mu \sigma) q^{\prime} / 2} d y\right)^{1 / q^{\prime}}\|W\|_{q} .
$$

From (4.14) - (4.16), the estimate of $\mathfrak{A}^{(2)}(r)$ mentioned above and (4.3), and because $(|x| \nu(x)) \geq R_{0}$, we obtain

$$
\int_{B_{R_{1}}}\left|\partial_{x}^{\alpha} \partial_{r}^{\sigma} \partial_{y}^{\beta} \mathfrak{G}_{j k}(x, y, t) W_{k}(y)\right| d y \leq C\left(R_{0}, S_{0}, q, \tau\right)\left|\nabla \varphi_{0}\right|_{\infty}(|x| \nu(x))^{-(3+|\alpha|+\sigma) / 2}\|W\|_{q} .
$$

This is inequality (4.12) if we take $r=t$ and $W=V$. Moreover, the choice $r=t-s, W=$ $v(s)$ and an integration with respect to $s$ yields (4.13) in the case $p=1$. Now suppose that $p \in(1, \infty]$. Then from $(4.14)-(4.16)$ and the estimate of $\mathfrak{A}^{(2)}(r)$ mentioned above with $r=t-s, W=v(s)$, we may conclude that the left-hand side of (4.13) is bounded by $C\left(R_{0}, S_{0}, q, \tau\right)\left|\nabla \varphi_{0}\right|_{\infty}$ times

$$
\sum_{\gamma \in\{0, \beta\}} \sum_{\mu=1}^{2}\left(\int_{0}^{t}\left(\int_{B_{R_{1}}} K(x-y, t-s)^{-(3+|\alpha|+|\gamma|+\mu \sigma) q^{\prime} / 2} d y\right)^{p^{\prime} / q^{\prime}} d s\right)^{1 / p^{\prime}}\|u\|_{q, p ; t} .
$$


Thus inequality (4.13) follows with (4.2).

Corollary 4.2 Let $\beta \in \mathbb{N}_{0}^{3}, \sigma \in\{0,1\}$ with $|\beta|+\sigma \leq 1$. Let $q \in(1, \infty)$, and let the function $v$ belong to $L_{\text {loc }}^{1}\left([0, \infty), L^{q}\left(B_{R_{1}}\right)^{3}\right)$ and the function $V$ to $L^{q}\left(B_{R_{1}}\right)^{3}$. For $x \in$ ${\overline{B_{R_{0}}}}^{c}, t \in[0, \infty)$, define

$$
F(x, t):=\int_{0}^{t} \int_{B_{R_{1}}} \partial_{s}^{\sigma} \partial_{y}^{\beta} \mathfrak{G}(x, y, t-s) \cdot v(y, s) d y d s, \quad H(x, t):=\int_{B_{R_{1}}} \mathfrak{G}(x, y, t) \cdot V(y) d y .
$$

Let $l \in\{1,2,3\}$. Then the derivatives $\partial x_{l} F(x, t)$ and $\partial x_{l} H(x, t)$ exist pointwise and equal $\int_{0}^{t} \int_{B_{R_{1}}} \partial x_{l} \partial_{s}^{\sigma} \partial_{y}^{\beta} \mathfrak{G}(x, y, t-s) \cdot v(y, s) d y d s$ and $\int_{B_{R_{1}}} \partial x_{l} \mathfrak{G}(x, y, t) \cdot V(y) d y$, respectively $\left(x \in \overline{B_{R_{0}}} c, t \in[0, \infty)\right)$.

Proof: Let $t \in[0, \infty)$ and define $\mathcal{L}, \mathcal{M}:{\overline{B_{R_{0}}}}^{c} \times(0, t) \mapsto L^{q^{\prime}}\left(B_{R_{1}}\right)^{3}$ by $\mathcal{L}(x, s)(y):=$ $\partial_{s}^{\sigma} \partial_{y}^{\beta} \mathfrak{G}(x, y, t-s), \mathcal{M}(x, s)(y):=\partial x_{l} \partial_{s}^{\sigma} \partial_{y}^{\beta} \mathfrak{G}(x, y, t-s)$ for $x \in{\overline{B_{R_{0}}}}^{c}, y \in B_{R_{1}}, s \in[0, t] ;$ see Lemma 3.12 and 3.13. Then $\left\|\left(\mathcal{L}\left(x+h e_{l}, s\right)-\mathcal{L}(x, s)\right) / h-\mathcal{M}(x, s)\right\|_{q^{\prime}} \rightarrow 0(h \rightarrow 0)$ for $x \in \overline{B_{R_{0}}}$, uniformly in $s \in[0, t]$, as follows from Lemma 3.13. The corollary follows from this and Hölder's inequality.

It will be convenient to subsume a number of terms in a single operator, which we define here. We recall that the parameters $T_{0}, S_{0}, R_{0}, R_{1}$ and the set $\Omega$ were fixed at the beginning of Section 2. Also the notation $\Omega_{R}$ for $R \in\left[S_{0}, \infty\right)$ and $n^{(\Omega)}$ were introduced there.

Put $Z_{R, T}:=\Omega_{R} \times(0, T)$ for $R \in\left[S_{0}, \infty\right), T \in(0, \infty]$. Let $\mathfrak{A} \subset \mathbb{R}^{3} \times \mathbb{R}$ with $Z_{R_{1}, T_{0}} \subset \mathfrak{A}$. Take $q \in(1, \infty)$ and suppose that $v: \mathfrak{A} \mapsto \mathbb{R}^{3}$ is a function such that $v \mid Z_{R_{1}, T_{0}}$ belongs to $C^{0}\left(\left[0, T_{0}\right), L^{q}\left(\Omega_{R_{1}}\right)^{3}\right), v(s) \mid \Omega_{R_{1}} \in W_{l o c}^{1,1}\left(\Omega_{R_{1}}\right)^{3}$ for $s \in\left(0, T_{0}\right)$, and $\nabla_{x} v \mid Z_{R_{1}, T_{0}} \in$ $L_{\text {loc }}^{1}\left(\left[0, T_{0}\right), L^{q}\left(\Omega_{R_{1}}\right)^{9}\right)$. Then, for $t \in\left(0, T_{0}\right)$ and $x \in B_{R_{0}}^{c}$, we define

$$
\begin{aligned}
& \mathfrak{K}_{R_{0}, S_{0}, \varphi_{0}, \Omega, T_{0}}(v)(x, t):=\int_{0}^{t} \int_{\Omega_{R_{1}}}\left(\sum_{l=1}^{3} \partial y_{l} \mathfrak{G}(x, y, t-s) \cdot \partial_{y_{l}} v(y, s)\right. \\
& \left.-\tau \partial y_{1} \mathfrak{G}(x, y, t-s) \cdot v(y, s)-\partial_{s} \mathfrak{G}(x, y, t-s) \cdot v(y, s)\right) d y d s+\int_{\Omega_{R_{1}}} \mathfrak{G}(x, y, 0) \cdot v(y, t) d y .
\end{aligned}
$$

The ensuing corollary provides a decay estimate of $\mathfrak{K}_{R_{0}, S_{0}, \varphi_{0}, \Omega, T_{0}}(v)$. We use the same notation as in (4.17).

Corollary 4.3 Let $q \in(1, \infty), p_{1}, p_{2} \in[1, \infty]$. Then, for functions $v: \mathfrak{A} \mapsto \mathbb{R}^{3}$ satisfying the relations $v\left|Z_{R_{1}, T_{0}} \in C^{0}\left(\left[0, T_{0}\right), L^{q}\left(\Omega_{R_{1}}\right)^{3}\right), v(s)\right| \Omega_{R_{1}} \in W_{l o c}^{1,1}\left(\Omega_{R_{1}}\right)^{3}$ for $s \in\left(0, T_{0}\right)$, and $\nabla_{x} v \mid Z_{R_{1}, T_{0}} \in L^{p_{2}}\left(0, T_{0}, L^{q}\left(\Omega_{R_{1}}\right)^{9}\right)$, and for $x \in{\overline{B_{R_{0}}}}^{c}, t \in\left(0, T_{0}\right), \alpha \in \mathbb{N}_{0}^{3}$ with $|\alpha| \leq 1$, the term $\left|\partial_{x}^{\alpha} \mathfrak{K}_{R_{0}, S_{0}, \varphi_{0}, \Omega, T_{0}}(v)(x, t)\right|$ is bounded by

$$
\mathfrak{C}\left(\left\|v\left|Z_{R_{1}, t}\left\|_{q, p_{1} ; t}+\right\| \nabla_{x} v\right| Z_{R_{1}, t}\right\|_{q, p_{2} ; t}+\left\|v(t) \mid \Omega_{R_{1}}\right\|_{q}\right) \max _{j \in\{1,2\}}(|x| \nu(x))^{-(3+|\alpha|) / 2+1 /\left(2 p_{j}^{\prime}\right)} .
$$

Proof: Corollary 4.2, Theorem 4.2 .

In the rest of this section, we discuss spatial decay of some other potential functions which will play a role in the representation formula introduced in Section 5; see (5.7). We begin 
by considering $\mathfrak{I}^{(\tau)}(U)$, in the case that $U$ has compact support. Then $\mathfrak{I}^{(\tau)}(U)$ turns out to decrease as rapidly for $|x| \rightarrow \infty$ as does the fundamental solution $\Lambda$ of (1.1).

Lemma 4.1 Let $V \in L^{1}\left(\mathbb{R}^{3}\right)^{3}$, and suppose there is some $\widetilde{R}>0$ with $\operatorname{supp}(V) \subset B_{\widetilde{R}}$. Let $R \in(\widetilde{R}, \infty), x \in B_{R}^{c}, t \in(0, \infty), \alpha \in \mathbb{N}_{0}^{3}$ with $|\alpha| \leq 1$. Then $\left|\partial_{x}^{\alpha} \mathfrak{I}^{(\tau)}(V)(x, t)\right| \leq$ $C(\widetilde{R}, R, \tau)\|V\|_{1}(|x| \nu(x))^{-(3+|\alpha|) / 2}$.

Proof: Corollary 3.5 yields that $\left|\partial_{x}^{\alpha} \mathfrak{I}^{(\tau)}(V)(x, t)\right| \leq \int_{B_{\tilde{R}}}\left|\partial_{x}^{\alpha} \Lambda(x-y, t) \cdot V(y)\right| d y$. By the first inequality in Corollary 3.3 and by Corollary 3.1 with $K=R-\widetilde{R}$, the right-hand side of this estimate is bounded by $C(\widetilde{R}, R, \tau)\|V\|_{1}(|x| \nu(x))^{-(3+|\alpha|) / 2}$.

Lemma 4.2 Let $f \in L^{1}\left(\mathbb{R}^{3} \times(0, \infty)\right)^{3}$ such that there is $\widetilde{R} \in(0, \infty)$ with supp $(f(s)) \subset$ $B_{\widetilde{R}}$ for any $s \in(0, \infty)$. Let $R \in(\widetilde{R}, \infty)$. Then

$$
\int_{0}^{t} \int_{\mathbb{R}^{3}}\left|\partial_{x}^{\alpha} \Lambda(x-y, t-s) \cdot f(y, s)\right| d y d s \leq C(\tau, \widetilde{R}, R)\|f\|_{1}(|x| \nu(x))^{-(3+|\alpha|) / 2}
$$

for $t \in(0, \infty), \quad x \in B_{R}^{c}, \alpha \in \mathbb{N}_{0}^{3}$ with $|\alpha| \leq 1$. For any such $t$ and $x$, the function $\mathfrak{R}^{(\tau)}(f)$ is defined in $(x, t)$ as stated in Lemma 3.8, and $\mathfrak{R}^{(\tau)}(f)(t)$ belongs to $W_{\text {loc }}^{1,1}\left(\mathbb{R}^{3}\right)^{3}$, with $\partial x_{l} \Re^{(\tau)}(f)(x, t)=\int_{0}^{t} \int_{\mathbb{R}^{3}} \partial_{x}^{\alpha} \Lambda(x-y, t-s) \cdot f(y, s) d y d s$ for $1 \leq l \leq 3$. (Lemma 3.8 yields these relations only for $a$. e. $t$ and a. e. $x$.) Thus $\left|\partial_{x}^{\alpha} \mathfrak{R}^{(\tau)}(f)(x, t)\right| \leq$ $C(\tau, \widetilde{R}, R)\|f\|_{1}(|x| \nu(x))^{-(3+|\alpha|) / 2}$ for $t, x, \alpha$ as in (4.18).

Proof: Applying the first inequality in Corollary 3.3 and Corollary 3.1 with $K=R-\widetilde{R}$, we get that $\left|\partial_{x}^{\alpha} \Lambda(x-y, t-s) \cdot f(y, s)\right| \leq C(\widetilde{R}, R, \tau)(|x| \nu(x))^{-(3+|\alpha|) / 2}|f(y, s)|$ for $t, x, \alpha$ as in (4.18), $y \in B_{\widetilde{R}}$ and $s \in(0, t)$. This estimate implies (4.18). Since $f \in L^{1}\left(\mathbb{R}^{3} \times(0, \infty)\right)^{3}$, the latter inequality and Lebesgue's theorem yield the remaining statements of Lemma 4.2 .

Lemma 4.3 Let $Z_{R_{1}, T}$ for $T \in\left(0, T_{0}\right]$ and $\mathfrak{A}$ be given as in (4.17). Take $q \in(1, \infty)$ and $p_{1}, p_{2} \in[1, \infty]$. Then, for $v: \mathfrak{A} \mapsto \mathbb{R}^{3}$ with $v\left|Z_{R_{1}, T_{0}} \in L^{p_{1}}\left(0, T_{0}, L^{q}\left(\Omega_{R_{1}}\right)^{3}\right), v(s)\right| \Omega_{R_{1}} \in$ $W_{l o c}^{1,1}\left(\Omega_{R_{1}}\right)^{3}$ for $s \in\left(0, T_{0}\right)$ and $\nabla_{x} v \mid Z_{R_{1}, T_{0}} \in L^{p_{2}}\left(0, T_{0}, L^{q}\left(\Omega_{R_{1}}\right)^{9}\right)$, as well as for $x \in$ $B_{R_{0}}^{c}, t \in\left(0, T_{0}\right), \alpha \in \mathbb{N}_{0}^{3}$ with $|\alpha| \leq 2, l \in\{1,2,3\}$, the term $\left|\partial_{x}^{\alpha} \mathfrak{V}^{(\tau)}\left(n_{l}^{(\Omega)} v\right)(x, t)\right|$ is bounded by

$$
\mathfrak{C}\left(\left\|v\left|Z_{R_{1}, t}\left\|_{q, p_{1} ; t}+\right\| \nabla_{x} v\right| Z_{R_{1}, t}\right\|_{q, p_{2} ; t}\right) \max _{j \in\{1,2\}}(|x| \nu(x))^{-(3+|\alpha|) / 2+1 /\left(2 p_{j}^{\prime}\right)},
$$

where $\left(n_{l}^{(\Omega)} v\right)(y, s):=n_{l}^{(\Omega)}(y) v(y, s)$ for $y \in \partial \Omega, s \in\left(0, T_{0}\right)$.

Proof: We use the function $\varphi_{0}$ introduced at the beginning of Section 2. Take $v, x, t, \alpha, l$ as in the lemma. Since $\varphi_{0} \in C_{0}^{\infty}\left(B_{R_{1}}\right)$ and $x \in B_{R_{0}}^{c}$, Lemma 3.3 yields that for $r \in$ $(0, \infty), \beta \in \mathbb{N}_{0}^{3}$, the function $y \mapsto \varphi_{0}(y) \partial_{x}^{\beta} \Lambda(x-y, r)\left(y \in B_{R_{1}}\right)$ belongs to $C_{0}^{\infty}\left(B_{R_{1}}\right)$. Combining the relations $\Omega \subset B_{S_{0}}$ and $\varphi_{0} \mid B_{S_{0}+\left(R_{0}-S_{0}\right) / 4}=1$ with Lemma 3.9 and the Divergence theorem, we may conclude that $\mathfrak{V}^{(\tau)}\left(\phi_{l, T_{0}}\right)(x, t)=A_{1}+A_{2}+A_{3}$, where $A_{1}:=\int_{0}^{t} \int_{\Omega_{R_{1}}} \partial_{x}^{\alpha} \partial y_{l} \Lambda(x-y, t-s) \cdot\left(\varphi_{0}(y) v(y, s)\right) d y d s, A_{2}$ stands for the double integral $\int_{0}^{t} \int_{\Omega_{R_{1}}} \partial_{x}^{\alpha} \Lambda(x-y, t-s) \cdot\left(\partial_{l} \varphi_{0}(y) v(y, s)\right) d y d s$, and $A_{3}$ is defined in the same 
way as $A_{2}$, but with the term $\partial_{l} \varphi_{0}(y) v(y, s)$ replaced by $\varphi_{0}(y) \partial y_{l} v(y, s)$. Corollary 4.1 implies that $A_{1}$ is bounded by $\mathfrak{C}\left\|v \mid Z_{R_{1}, t}\right\|_{q, p_{1} ; t}(|x| \nu(x))^{-(4+|\alpha|) / 2+1 /\left(2 p_{1}^{\prime}\right)}$, and $A_{2}$ by $\mathfrak{C}\left\|v \mid Z_{R_{1}, t}\right\|_{q, p_{1} ; t}(|x| \nu(x))^{-(3+|\alpha|) / 2+1 /\left(2 p_{1}^{\prime}\right)}$. As for $A_{3}$, we may again use Corollary 4.1, obtaining the upper bound $\mathfrak{C}\left\|\nabla_{x} v \mid Z_{R_{1}, t}\right\|_{q, p_{2} ; t}(|x| \nu(x))^{-(3+|\alpha|) / 2+1 /\left(2 p_{2}^{\prime}\right)}$. The lemma follows from these estimates.

Lemma 4.4 Let $q \in(1, \infty)$. Then the estimate $\left|\int_{\partial \Omega}\left(\partial^{\alpha} \nabla \mathfrak{N}\right)(x-y)\left(n^{(\Omega)} \cdot V\right)(y) d o_{y}\right| \leq$ $\mathfrak{C}\|V\|_{q}|x|^{-2-|\alpha|}$ holds for $V \in L^{q}\left(\Omega_{R_{1}}\right)^{3} \cap W^{1,1}\left(\Omega_{R_{1}}\right)^{3}$ with div $V=0, t \in(0, \infty), x \in$ $B_{R_{0}}^{c}, \alpha \in \mathbb{N}_{0}^{3}$ with $|\alpha| \leq 1$. If $\int_{\partial \Omega} n^{(\Omega)} \cdot V d o_{y}=0$, the factor $|x|^{-2-|\alpha|}$ may be replaced by $|x|^{-3-|\alpha|}$. (The Newton kernel $\mathfrak{N}$ was defined at the beginning of Section 3.)

Proof: We again use the function $\varphi_{0}$ introduced at the beginning of Section 2. Let $V, x, t, \alpha$ be given as in the lemma, and take $j \in\{1,2,3\}$. Since $x \in B_{R_{0}}^{c}$ and $\varphi_{0} \in$ $C_{0}^{\infty}\left(B_{R_{1}}\right)$, the function $y \mapsto \partial_{j} \mathfrak{N}(x-y) \varphi_{0}(y)\left(y \in B_{R_{1}}\right)$ belongs to $C_{0}^{\infty}\left(B_{R_{1}}\right)$. This fact, the relations $\operatorname{div} V=0, \Omega \subset B_{S_{0}}, \varphi_{0} \mid B_{S_{0}+\left(R_{0}-S_{0}\right) / 4}=1, S_{0}<R_{1}$ and the Divergence theorem yield that $\mathfrak{A}:=\int_{\partial \Omega}\left(\partial^{\alpha} \partial_{j} \mathfrak{N}\right)(x-y)\left(n^{(\Omega)} \cdot V\right)(y) d o_{y}$ coincides with $-\int_{\Omega_{R_{1}}}\left[\nabla_{y}\left(\partial^{\alpha} \partial_{j} \mathfrak{N}\right)(x-y) \cdot\left(\varphi_{0} V\right)(y)+\left(\partial^{\alpha} \partial_{j} \mathfrak{N}\right)(x-y)\left(\nabla \varphi_{0} \cdot V\right)(y)\right] d y$. Since $\left|\left(\nabla \partial^{\beta} \mathfrak{N}\right)(z)\right| \leq$ $C|z|^{-2-|\beta|}$ for $z \in \mathbb{R}^{3} \backslash\{0\}, \beta \in \mathbb{N}_{0}^{3}$ with $|\beta| \leq 2$, and because $\operatorname{supp}\left(\varphi_{0}\right) \subset B_{R_{1}}$ and $|x-y| \geq\left(1-R_{1} / R_{0}\right)|x|$ for $y \in B_{R_{1}}$, we may conclude that the term $|\mathfrak{A}|$ is bounded by $C\left(S_{0}, R_{0}\right)\|V\|_{1}\left(|x|^{-3-|\alpha|}+|x|^{-2-|\alpha|}\left|\nabla \varphi_{0}\right|_{\infty}\right)$. The first part of the lemma follows from this estimate.

Now suppose that $\int_{\partial \Omega} n^{(\Omega)} \cdot V d o_{y}=0$. Put $A:=A_{R_{1}, S_{0}}$. Since $\Omega \subset B_{S_{0}}, S_{0}<R_{1}, \varphi_{0} \in$ $C_{0}^{\infty}\left(B_{R_{1}}\right)$ and $\varphi \mid B_{S_{0}+\left(R_{0}-S_{0}\right) / 4}=1$, we conclude that $\int_{A} \nabla \varphi_{0} \cdot V d o_{y}=0$. Therefore we may apply the operator $\mathfrak{D}:=\mathfrak{D}_{R_{1}, S_{0}}$ from Theorem 2.4 to $\nabla \varphi_{0} \cdot V \mid A$, obtaining that the function $F:=\mathfrak{D}\left(\nabla \varphi_{0} \cdot V \mid A\right)$ belongs to $W_{0}^{1, q}(A)^{3}$ and $\operatorname{div}\left(\varphi_{0} V-F\right)=0$. Now the Divergence theorem yields $\mathfrak{A}=-\int_{\Omega_{R_{1}}} \nabla_{y}\left(\partial^{\alpha} \partial_{j} \mathfrak{N}\right)(x-y) \cdot\left(\varphi_{0} V-F\right)(y) d y$. But $\operatorname{supp}\left(\varphi_{0} V-F\right) \subset B_{R_{1}}$ and $|x-y| \geq\left(1-R_{1} / R_{0}\right)|x|$ for $y \in B_{R_{1}}$, so it follows that $|\mathfrak{A}| \leq C\left(S_{0}, R_{0}\right)|x|^{-3-|\alpha|}\left\|\varphi_{0} V-F\right\|_{1}$. On the other hand, we get with Theorem 2.4 that $\left\|\varphi_{0} V-F\right\|_{1} \leq C\left(S_{0}, R_{0}, q\right)\left(1+\left|\nabla \varphi_{0}\right|_{\infty}\right)\|V\| q$. Combining these estimates yields the second claim of the lemma.

\section{A representation formula for solutions to (1.1).}

In present section, we derive the integral representation (equation (5.7)) announced in Section 1. Our general approach follows the one used in [15, Section 4]. However, we are going to treat a more general framework and the Oseen system instead of the Stokes system, so there is some extra work to do. Recall that the parameters $T_{0}, S_{0}, R_{0}, R_{1}$, the function $\varphi_{0}$ and the set $\Omega$ were introduced at the beginning of Section 2 .

Lemma 5.1 Let $q \in(1, \infty), u \in W_{l o c}^{1,1}\left(\left[0, T_{0}\right), L^{q}\left(\bar{\Omega}^{c}\right)^{3}\right) \cap C^{0}\left(\left[0, T_{0}\right), L^{q}\left(\bar{\Omega}^{c}\right)^{3}\right)$. Take 
$t \in\left(0, T_{0}\right), \epsilon \in(0, t)$ and $x \in \mathbb{R}^{3}$. Then, with $A$ introduced in (3.4),

$$
\begin{aligned}
& \int_{0}^{t-\epsilon} \int_{\bar{\Omega}^{c}}\left(\Lambda(x-y, t-s) \cdot u^{\prime}(s)(y)+\partial_{s} \Lambda(x-y, t-s) \cdot u(y, s)\right) d y d s \\
& =\int_{\bar{\Omega}^{c}} \Lambda(x-y, \epsilon) \cdot u(y, t-\epsilon) d y-\mathfrak{I}^{(\tau)}(u(0))(x, t) .
\end{aligned}
$$

Proof: According to (3.8), the estimate $\int_{\bar{\Omega}^{c}}\left|\partial_{s}^{\sigma} \Lambda(x-y, t-s) \cdot V(y)\right| d y \leq \mathfrak{C}(\epsilon)\|V\|_{q}$ holds for $\sigma \in\{0,1\}, V \in L^{q}\left(\bar{\Omega}^{c}\right)^{3}$ and $s \in(0, t-\epsilon)$. Therefore, for such $\sigma$, we may define an mapping $G^{(\sigma)}:(0, t-\epsilon) \mapsto\left[L^{q}\left(\bar{\Omega}^{c}\right)^{3}\right]^{\prime}=L^{q^{\prime}}\left(\bar{\Omega}^{c}\right)^{3}$ by setting $G^{(\sigma)}(s)(V):=$ $\int_{\bar{\Omega}^{c}} \partial_{s}^{\sigma} \Lambda(x-y, t-s) \cdot V(y) d y$ for $V, s$ as before. Then $G^{(\sigma)} \in L^{1}\left(0, t-\epsilon,\left[L^{q}\left(\bar{\Omega}^{c}\right)^{3}\right]^{\prime}\right)$. In addition, the estimate above allows us to apply Fubini's theorem, which yields that $\int_{0}^{t-\epsilon} \psi(s) G^{(1)}(s)(V) d s=-\int_{0}^{t-\epsilon} \psi^{\prime}(s) G^{(0)}(s)(V) d s$ for $\psi \in C_{0}^{\infty}((0, t-\epsilon)), V \in L^{q}\left(\bar{\Omega}^{c}\right)^{3}$. As a consequence (Theorem 2.5), $\int_{0}^{t-\epsilon} \psi(s) G^{(1)}(s) d s=-\int_{0}^{t-\epsilon} \psi^{\prime}(s) G^{(0)}(s) d s$ for $\psi$ as before, where the integrals are $\left[L^{q}\left(\bar{\Omega}^{c}\right)^{3}\right]^{\prime}$-valued Bochner integrals. Thus we obtain that $G^{(0)} \in W^{1,1}\left(0, t-\epsilon,\left[L^{q}\left(\bar{\Omega}^{c}\right)^{3}\right]^{\prime}\right)$ with $\left(G^{(0)}\right)^{\prime}=G^{(1)}$. The estimate at the end of Corollary 3.6 yields that $G^{(0)}$ is continuous as a mapping from $(0, t-\epsilon)$ into $\left[L^{q}\left(\bar{\Omega}^{c}\right)^{3}\right]^{\prime}$. Now Lemma 2.5 and the assumptions on $u$ yield $\int_{0}^{t-\epsilon}\left[G^{(0)}(s)\left(u^{\prime}(s)\right)+G^{(1)}(s)(u(s))\right] d s=$ $G^{(0)}(t-\epsilon)(u(t-\epsilon))-G^{(0)}(0)(u(0))$. Hence the lemma follows from the definition of $G^{(0)}$ and $G^{(1)}$.

Theorem 5.1 Let $q \in(1, \infty)$ and $u:\left[0, T_{0}\right) \mapsto L_{l o c}^{q}\left(\bar{\Omega}^{c}\right)^{3}$ a function such that $u(s) \mid \Omega_{R} \in$ $W^{1,1}\left(\Omega_{R}\right)^{3}\left(R \in\left[S_{0}, \infty\right)\right)$ and div $x u(s)=0$ for $s \in\left(0, T_{0}\right) \backslash \mathfrak{T}_{T_{0}}$, where $\mathfrak{T}_{T_{0}} \subset\left(0, T_{0}\right)$ is a set of measure zero. Further suppose that $u \mid \Omega_{R} \times\left[0, T_{0}\right) \in C^{0}\left(\left[0, T_{0}\right), L^{q}\left(\Omega_{R}\right)^{3}\right)$ for any $R \in\left[S_{0}, \infty\right)$. Let $t \in\left(0, T_{0}\right) \backslash \mathfrak{T}_{T_{0}}, x \in \bar{\Omega}^{c}$. Then

$\int_{\partial \Omega} A\left(x-y-\tau \epsilon e_{1}, \epsilon\right)\left(n^{(\Omega)}(y) \cdot u(y, t-\epsilon)\right) d o_{y} \rightarrow \int_{\partial \Omega}(\nabla \mathfrak{N})(x-y)\left(n^{(\Omega)}(y) \cdot u(y, t)\right) d o_{y}$

for $\epsilon \rightarrow 0, t-\epsilon \in(0, t) \backslash \mathfrak{T}_{T_{0}}$. (The function $\mathfrak{N}$ (Newton kernel) was introduced in at the beginning of Section 3, and the function $A$ in (3.4).)

Proof: Since $x \in \bar{\Omega}^{c}$, we have $\delta:=\operatorname{dist}(x, \bar{\Omega})>0$. Let $R \in\left[S_{0}, \infty\right)$ with $\overline{B_{\delta}(x)} \subset B_{R}$. We may choose a function $\psi=\psi_{x} \in C_{0}^{\infty}\left(B_{2 R}\right)$ with $\psi \mid B_{R} \backslash B_{\delta / 2}(x)=1$ and $\psi \mid B_{\delta / 4}(x)=0$. This means in particular that $\psi \mid \partial \Omega=1, \operatorname{supp}(\psi) \subset B_{2 R} \backslash B_{\delta / 4}(x)$, and $|x-y| \geq \delta / 4$ for any $y \in \operatorname{supp}(\psi)$. Put $U_{x}:=\Omega_{2 R} \backslash \overline{B_{\delta / 4}(x)}$. Let $j \in\{1,2,3\}$, and define $\mathfrak{A}_{\epsilon}:=$ $\int_{\partial \Omega} A_{j}\left(x-y-\tau \epsilon e_{1}, \epsilon\right)\left(n^{(\Omega)}(y) \cdot u(y, t-\epsilon)\right) d o_{y}$ for $\epsilon \in(0, t)$. Then by the Divergence theorem, Lemma 3.5, the choice of $\psi$, and because $\operatorname{div}_{y} u(t-\epsilon)=0$ for $\epsilon \in(0, t)$ with $t-\epsilon \in(0, t) \backslash \mathfrak{T}_{T_{0}}$, we get $\mathfrak{A}_{\epsilon}=\int_{U_{x}} \int_{\epsilon}^{\infty} \mathfrak{P}(y, s, \epsilon) d s d y$ for $\epsilon$ as before, where

$$
\begin{aligned}
& \mathfrak{P}(y, s, \epsilon):=-\nabla_{y} \partial x_{j} \mathfrak{H}\left(x-y-\tau \epsilon e_{1}, s\right) \cdot \psi(y) u(y, t-\epsilon) \\
& \quad-\partial x_{j} \mathfrak{H}\left(x-y-\tau \epsilon e_{1}, s\right) \nabla \psi(y) \cdot u(y, t-\epsilon) \text { for } \epsilon \in[0, t], y \in U_{x}, s \in(0, \infty) .
\end{aligned}
$$

The minus sign arises because $n^{(\Omega)}$ is the outward unit normal to $\Omega$. We split $\mathfrak{A}_{\epsilon}$ into a sum $\mathfrak{A}_{\epsilon}^{(1)}+\mathfrak{A}_{\epsilon}^{(2)}$, with $\mathfrak{A}_{\epsilon}^{(j)}:=\int_{U_{x}} \int_{\epsilon}^{\infty} \mathfrak{P}^{(j)}(y, s, \epsilon) d s d y\left(\epsilon \in(0, t), t-\epsilon \notin \mathfrak{T}_{T_{0}}\right)$ where $\mathfrak{P}^{(j)}(y, s, \epsilon)$ for $j \in\{1,2\}$ is defined in the same way as $\mathfrak{P}(y, s, \epsilon),(y, s, \epsilon$ as in that definition), except that the term $u(y, t-\epsilon)$ is replaced by $u(y, t-\epsilon)-u(y, t)$ in the case 
$j=1$, and by $u(y, t)$ if $j=2$. For $y \in U_{x}, s \in(0, \infty), \epsilon \in[0, t]$, put $Q(y, s, \epsilon):=$ $\left(\sum_{k=1}^{3}\left|\partial x_{k} \partial x_{j} \mathfrak{H}\left(x-y-\tau \epsilon e_{1}, s\right)\right|\right)+\left|\partial x_{j} \mathfrak{H}\left(x-y-\tau \epsilon e_{1}, s\right)\right|$. Note that

$\left|\mathfrak{P}^{(1)}(y, s, \epsilon)\right| \leq \mathfrak{C} Q(y, s, \epsilon)|u(y, t-\epsilon)-u(y, t)|, \quad\left|\mathfrak{P}^{(2)}(y, s, \epsilon)\right| \leq \mathfrak{C} Q(y, s, \epsilon)|u(y, t)|$

for $y, s, \epsilon$ as in the definition of $Q(y, s, \epsilon)$. Since $|x-y| \geq \delta / 4$ for $y \in U_{x}$, as mentioned above, and because of Theorem 3.1, we find for $y \in U_{x}, s \in(0, \infty), \epsilon \in[0, \min \{t, \delta /(8 \tau)\}]$ that

$$
Q(y, s, \epsilon) \leq \mathfrak{C} \max _{\mu \in\{0,1\}}\left(\left|x-y-\tau \epsilon e_{1}\right|^{2}+s\right)^{-2-\mu / 2} \leq \mathfrak{C} \max _{\mu \in\{0,1\}}\left(\delta^{2}+s\right)^{-2-\mu / 2} .
$$

Thus from (5.1) and the definition of $\mathfrak{A}_{\epsilon}^{(1)}$, for $\epsilon \in(0, \min \{t, \delta /(8 \tau)\})$ with $t-\epsilon \notin \mathfrak{T}_{T_{0}}$,

$$
\left|\mathfrak{A}_{\epsilon}^{(1)}\right| \leq \mathfrak{C} \max _{\mu \in\{0,1\}} \int_{U_{x}} \int_{\epsilon}^{\infty}\left(\delta^{2}+s\right)^{-2-\mu / 2}|u(y, t-\epsilon)-u(y, t)| d s d y
$$

hence $\left|\mathfrak{A}_{\epsilon}^{(1)}\right|$ is bounded by $\mathfrak{C}(\delta, R)\left\|u(t-\epsilon)-u(t) \mid \Omega_{2 R}\right\|_{q}$. Since $u \mid \Omega_{2 R} \times\left[0, T_{0}\right)$ belongs to $C^{0}\left(\left[0, T_{0}\right), L^{q}\left(\Omega_{2 R}\right)^{3}\right)$, we may conclude that $\mathfrak{A}_{\epsilon}^{(1)} \rightarrow 0\left(\epsilon \rightarrow 0, t-\epsilon \in(0, t) \backslash \mathfrak{T}_{T_{0}}\right)$. Moreover, again using (5.1) and (5.2), we get $\left|\mathfrak{P}^{(2)}(y, s, \epsilon)\right| \leq \mathfrak{C} \max _{\mu \in\{0,1\}}\left(\delta^{2}+s\right)^{-2-\mu / 2}|u(y, t)|$ for $y \in U_{x}, s \in(0, \infty), \epsilon \in[0, \min \{t, \delta /(8 \tau)\}]$. The right-hand side of this estimate is independent of $\epsilon$ and constitutes an integrable function of $(y, s) \in U_{x} \times(0, \infty)$. Note that $u(t) \mid \Omega_{2 R} \in L^{q}\left(\Omega_{2 R}\right)^{3}$. We further remark that $\chi_{(\epsilon, \infty)}(s) \mathfrak{P}^{(2)}(y, s, \epsilon) \rightarrow \mathfrak{P}^{(2)}(y, s, 0)(\epsilon \downarrow 0)$ for $s \in(0, \infty), y \in U_{x}$. Thus Lebesgue's theorem and the definition of $\mathfrak{A}_{\epsilon}^{(2)}$ yield that $\int_{U_{x}} \int_{0}^{\infty}\left|\mathfrak{P}^{(2)}(y, s, 0)\right| d s d y<\infty$ and $\mathfrak{A}_{\epsilon}^{(2)} \rightarrow \int_{U_{x}} \int_{0}^{\infty} \mathfrak{P}^{(2)}(y, s, 0) d s d y(\epsilon \rightarrow 0, t-\epsilon \in$ $\left.(0, t) \backslash \mathfrak{T}_{T_{0}}\right)$. This relation may be rewritten as

$$
\mathfrak{A}_{\epsilon}^{(2)} \rightarrow \int_{U_{x}}[-\nabla \mathfrak{B}(y) \cdot \psi(y) u(y, t)-\mathfrak{B}(y) \nabla \psi(y) \cdot u(y, t)] d y
$$

for $\epsilon \rightarrow 0, t-\epsilon \in(0, t) \backslash \mathfrak{T}_{T_{0}}$, where $\mathfrak{B}(y):=\int_{0}^{\infty} \partial x_{j} \mathfrak{H}(x-y, s) d s$ for $y \in U_{x}$. But this latter integral may be computed. It turns out to be equal to $\left(\partial_{j} \mathfrak{N}\right)(x-y)$. At this point, we recall that $\mathfrak{A}_{\epsilon}=\int_{U_{x}} \int_{\epsilon}^{\infty} \mathfrak{P}(y, s, \epsilon) d s d y$ and $\mathfrak{A}_{\epsilon}=\mathfrak{A}_{\epsilon}^{(1)}+\mathfrak{A}_{\epsilon}^{(2)}$ for $\epsilon \in(0, t), t-\epsilon \notin \mathfrak{T}_{T_{0}}$. We further recall that $\mathfrak{A}_{\epsilon}^{(1)} \rightarrow 0\left(\epsilon \rightarrow 0, t-\epsilon \in(0, t) \backslash \mathfrak{T}_{T_{0}}\right)$ as shown further above, and that $\operatorname{div}_{y} u(t)=0$ by our assumptions on $t$. Thus the theorem follows from (5.3) by another application of the Divergence theorem.

The boundary integrals appearing in the following lemma are not defined by some trace theorem, but correspond to lower order integrals in Fubini's theorem.

Lemma 5.2 Let $m \in \mathbb{N}, p_{j} \in(1, \infty), V^{(j)} \in L_{\text {loc }}^{1}\left({\overline{B_{R_{0}}}}^{c}\right)$ for $1 \leq j \leq m$, and suppose that $\int_{\overline{B_{R_{0}}}} c\left[\left|V^{(j)}(x)\right| /((1+|x|) \ln (2+|x|))\right]^{p_{j}} d o_{x}<\infty$ for such $j$. Let $\beta \in \mathbb{N}_{0}^{3}$ with $|\beta| \leq 1$, and take $s \in(0, \infty)$. Then there is a sequence $\left(R_{n}\right)$ in $\left[R_{0}, \infty\right)$ such that $R_{n} \rightarrow \infty$ and $\sum_{k, l=1}^{3} \sum_{j=1}^{m} \int_{\partial B_{R_{n}}}\left|\partial_{y}^{\beta} \Lambda_{k l}(x-y, s) V^{(j)}(y)\right| d o_{y} \rightarrow 0$ for $n \rightarrow \infty$.

Proof: Put $\widetilde{V}^{(j)}(y):=\left|V^{(j)}(y)\right| /((1+|y|) \ln (2+|y|))$ for $y \in{\overline{B_{R_{0}}}}^{c}, j \in\{1, \ldots, m\}$. Suppose there is $\widetilde{R} \in\left[R_{0}, \infty\right)$ with $\sum_{j=1}^{m} \int_{\partial B_{r}} \widetilde{V}^{(j)}(y)^{p_{j}} d o_{y} \geq 1 / r$ for $r \in[\widetilde{R}, \infty)$. Then 
$\sum_{j=1}^{m} \int_{\bar{B}_{\widetilde{R}}} \widetilde{V}^{(j)}(y)^{p_{j}} d y=\sum_{j=1}^{m} \int_{\widetilde{R}}^{\infty} \int_{\partial B_{r}} \widetilde{V}^{(j)}(y)^{p_{j}} d o_{y} d r=\infty$. Since $\widetilde{R} \geq R_{0}$, this is a contradiction to the assumption $\int_{\bar{B}_{R_{0}}} c \widetilde{V}^{(j)}(x)^{p_{j}} d x<\infty$ for $1 \leq j \leq m$. Thus we may choose a sequence $\left(R_{n}\right)$ in $\left[R_{0}, \infty\right)$ with $R_{n} \rightarrow \infty$ and $\sum_{j=1}^{m} \int_{\partial B_{R_{n}}} \widetilde{V}^{(j)}(y)^{p_{j}} d o_{y} \leq 1 / R_{n}$ for $n \in \mathbb{N}$. On the other hand, for $n \in \mathbb{N}$ with $R_{n} \geq \max \{1,2(|x|+s)\}$ and for $y \in \partial B_{R_{n}}$, with $s \in(0, \infty)$ fixed in the lemma, we get due to the first estimate in Corollary 3.3 that $\left|\partial_{y}^{\beta} \Lambda(x-y, s)\right| \leq \mathfrak{C}\left|x-y-\tau s e_{1}\right|^{-3-|\beta|} \leq \mathfrak{C}(|y|-(|x|+s))^{-3-|\beta|} \leq \mathfrak{C}|y|^{-3-|\beta|} \leq \mathfrak{C} R_{n}^{-3}$. Thus for such $n$,

$$
\begin{aligned}
& \sum_{k, l=1}^{3} \sum_{j=1}^{m} \int_{\partial B_{R_{n}}}\left|\partial_{y}^{\beta} \Lambda_{k l}(x-y, s) V^{(j)}(y)\right| d o_{y} \\
& \leq \sum_{k, l=1}^{3} \sum_{j=1}^{m}\left(\int_{\partial B_{R_{n}}}\left(\left|\partial_{y}^{\beta} \Lambda_{k l}(x-y, s)\right|(1+|y|) \ln (2+|y|)\right)^{p_{j}^{\prime}} d y\right)^{1 / p_{j}^{\prime}}\left\|\widetilde{V}^{(j)} \mid \partial B_{R_{n}}\right\|_{p_{j}} \\
& \leq \mathfrak{C} \sum_{j=1}^{m} R_{n}^{-2-1 / p_{j}} \ln \left(2+R_{n}\right)\left(\int_{\partial B_{R_{n}}} d o_{y}\right)^{1 / p_{j}^{\prime}} \leq \mathfrak{C} \sum_{j=1}^{m} R_{n}^{-3 / p_{j}} \ln \left(2+R_{n}\right) .
\end{aligned}
$$

The lemma follows from this estimate.

Now we are in a position to prove a first version of our representation formula. We note that in the ensuing theorem, the Oseen system (1.1) is rewritten as equation (5.4), better adapted than (1.1) to the type of solution we consider here.

Theorem 5.2 Let $k_{0} \in \mathbb{N}$ and $\varrho_{k} \in(1, \infty)$ for $1 \leq k \leq k_{0}$. For such $k$, let $u^{(k)}$ belong to $W_{l o c}^{1,1}\left(\left[0, T_{0}\right), L^{\varrho_{k}}\left(\bar{\Omega}^{c}\right)^{3}\right)$ and to $C^{0}\left(\left[0, T_{0}\right), L^{\varrho_{k}}\left(\bar{\Omega}^{c}\right)^{3}\right)$. Put $u:=\sum_{k=1}^{k_{0}} u^{(k)}$, and let $\pi:\left(0, T_{0}\right) \mapsto W_{l o c}^{1,1}\left(\bar{\Omega}^{c}\right)^{3}, n_{0} \in \mathbb{N}, p_{j} \in(1, \infty)$ and $f^{(j)} \in L_{l o c}^{1}\left(\left[0, T_{0}\right), L^{p_{j}}\left(\bar{\Omega}^{c}\right)^{3}\right)$ for $1 \leq$ $j \leq n_{0}$. Suppose that the following additional properties are satisfied: $u(s) \in W_{\text {loc }}^{2,1}\left(\bar{\Omega}^{c}\right)^{3}$,

$$
u^{\prime}(s)-\Delta_{x} u(s)+\tau \partial x_{1} u(s)+\nabla_{x} \pi(s)=f(s), \quad \operatorname{div}_{x} u(s)=0
$$

for a. e. $s \in\left(0, T_{0}\right)$, with $f:=\sum_{j=1}^{n_{0}} f^{(j)}, u^{\prime}:=\sum_{k=1}^{k_{0}}\left(u^{(k)}\right)^{\prime}$;

there is $q_{1} \in(1, \infty)$ such that $\nabla_{y} u(s) \mid \overline{B_{R_{0}}} \in L^{q_{1}}\left(\overline{B_{R_{0}}}\right)^{9}$ for a. e. $s \in\left(0, T_{0}\right)$,

$u\left|\Omega_{R_{0}} \times\left(0, T_{0}\right) \in L_{l o c}^{1}\left(\left[0, T_{0}\right), W^{2,1}\left(\Omega_{R_{0}}\right)^{3}\right), \pi\right| \Omega_{R_{0}} \times\left(0, T_{0}\right) \in L_{l o c}^{1}\left(\left[0, T_{0}\right), W^{1,1}\left(\Omega_{R_{0}}\right)\right) ;$

there are numbers $m_{0} \in \mathbb{N}, \gamma_{j} \in(1, \infty)$ as well as functions $\pi^{(j)}:\left(0, T_{0}\right) \mapsto L_{\text {loc }}^{1}\left(\overline{B_{R_{0}}}{ }^{c}\right)$ for $1 \leq j \leq m_{0}$ such that

$$
\pi(s) \mid{\overline{B_{R_{0}}}}^{c}=\sum_{j=1}^{m_{0}} \pi^{(j)}(s), \sum_{j=1}^{m_{0}} \int_{{\overline{B_{R_{0}}}}^{c}}\left(\left|\pi^{(j)}(y, s)\right|[(1+|y|) \ln (2+|y|)]^{-1}\right)^{\gamma_{j}} d y<\infty
$$

for for a. e. $s \in\left(0, T_{0}\right)$. Let $\mathfrak{T}_{T_{0}} \subset\left(0, T_{0}\right)$ be a zero-measure set such that $u(t) \mid \Omega_{R} \in$ $W^{1,1}\left(\Omega_{R}\right)^{3}\left(R \in\left[S_{0}, \infty\right)\right)$ and div $u(t)=0$ for $t \in\left(0, T_{0}\right) \backslash \mathfrak{T}_{T_{0}}$. (Such a set exists due to (5.4).) Let $t \in\left(0, T_{0}\right) \backslash \mathfrak{T}_{T_{0}}$. Then there is a zero-measure set $N_{t} \subset \bar{\Omega}^{c}$ such that for 
$x \in \bar{\Omega}^{c} \backslash N_{t}$,

$$
\begin{gathered}
u(x, t)=\mathfrak{R}^{(\tau)}(f)(x, t)+\mathfrak{I}^{(\tau)}(u(0))(x, t)-\sum_{l=1}^{3} \partial x_{l} \mathfrak{V}^{(\tau)}\left(n_{l}^{(\Omega)} u\right)(x, t) \\
+\mathfrak{V}^{(\tau)}\left(\tau n_{1}^{(\Omega)} u+\sum_{l=1}^{3} n_{l}^{(\Omega)}\left(-\partial y_{l} u+\pi e_{l}\right)\right)(x, t)-\int_{\partial \Omega}(\nabla \mathfrak{N})(x-y)\left(n^{(\Omega)}(y) \cdot u(y, t)\right) d o_{y},
\end{gathered}
$$

with $\mathfrak{N}$ denoting the Newton kernel as introduced at the beginning of Section 3.

Proof: We modify the proof of [15, Theorem 4.2]. Let $x \in \bar{\Omega}^{c}$. For a. e. $s \in(0, t)$ the relations in (5.4) and (5.5) hold, and $u^{(j)}(s),\left(u^{(j)}\right)^{\prime}(s) \in L^{\varrho_{j}}\left(\bar{\Omega}^{c}\right)^{3}$ for $1 \leq j \leq$ $k_{0}, \nabla_{y} u(s) \mid{\overline{B_{R_{0}}}}^{c} \in L^{q_{1}}\left({\overline{B_{R_{0}}}}^{c}\right)^{9}$, as well as $f^{(j)}(s) \in L^{p_{j}}\left(\bar{\Omega}^{c}\right)^{3}$ for $1 \leq j \leq n_{0}$. Take such a number $s \in(0, t)$. Then we may choose a sequence $\left(R_{n}\right)$ in $\left[R_{0}, \infty\right)$ as in Lemma 5.2 with $s$ replaced by $t-s$ and $V^{(k)}(y):=\left|u^{(k)}(y, s)\right|$ for $1 \leq k \leq k_{0}, V^{\left(k_{0}+l\right)}(y):=\left|\partial y_{l} u(y, s)\right|$ for $1 \leq l \leq 3, V^{\left(k_{0}+3+j\right)}(y):=\left|\pi^{(j)}(y, s)\right|$ for $1 \leq j \leq n_{0}, y \in{\overline{B_{R_{0}}}}^{c}$. By (5.4), we have $0=\int_{\Omega_{R_{n}}} \Lambda(x-y, t-s) \cdot\left(u^{\prime}-\Delta_{y} u+\tau \partial y_{1} u+\nabla_{y} \pi-f\right)(y, s) d y$ for $n \in \mathbb{N}$. Recalling that $n^{(\Omega)}$ denotes the outward unit normal to $\Omega$, we get by an integration by parts that $0=\mathfrak{A}_{1}(x, s)+\mathfrak{A}_{2}(x, s)+\sum_{\mu=3}^{7} \mathfrak{A}_{\mu}(n, x, s)$, with

$$
\begin{aligned}
& \mathfrak{A}_{1}(x, s) \\
& :=\int_{\partial \Omega} \Lambda(x-y, t-s) \cdot\left(\sum_{l=1}^{3} n_{l}^{(\Omega)}(y)\left(\partial y_{l} u(y, s)-\pi(y, s) e_{l}\right)-\tau n_{1}^{(\Omega)}(y) \cdot u(y, s)\right) d o_{y}, \\
& \mathfrak{A}_{2}(x, s):=-\int_{\partial \Omega} \sum_{l=1}^{3} \partial y_{l} \Lambda(x-y, t-s) \cdot n_{l}^{(\Omega)}(y) u(y, s) d o_{y}, \\
& \mathfrak{A}_{5}(n, x, s):=-\int_{\Omega_{R_{n}}}\left(-\Delta_{y}-\tau \partial y_{1}\right) \Lambda(x-y, t-s) \cdot u(y, s) d y \\
& \mathfrak{A}_{6}(n, x, s):=-\int_{\Omega_{R_{n}}} \Lambda(x-y, t-s) \cdot f(y, s) d y .
\end{aligned}
$$

The terms $\mathfrak{A}_{3}(n, x, s)$ and $\mathfrak{A}_{4}(n, x, s)$ are defined as $\mathfrak{A}_{1}(x, s)$ and $\mathfrak{A}_{2}(x, s)$, respectively, but with the domain of integration $\partial \Omega$ replaced by $\partial B_{R_{n}}$, and the factor $n_{l}^{(\Omega)}(y)$ by $-y_{l} / R_{n}$. The term $\mathfrak{A}_{7}(n, x, s)$ is defined as $\mathfrak{A}_{6}(n, x, s)$, but with $-u^{\prime}(y, s)$ in the place of $f(y, s)$. By Lemma 3.3, we have $\mathfrak{A}_{5}(n, x, s)=\int_{\Omega_{R_{n}}} \partial_{s} \Lambda(x-y, t-s) \cdot u(y, s) d y$ for $n \in \mathbb{N}$. Moreover, recall that by Corollary 3.4 and because $t-s>0$, the terms $\Lambda(x-y, t-s)$ and $\partial_{s} \Lambda(x-y, t-s)$ as functions of $y \in \mathbb{R}^{3}$ belong to $L^{p}\left(\mathbb{R}^{3}\right)^{3 \times 3}$ for any $p \in(1, \infty)$. From these observations we get that $\mathfrak{A}_{5}(n, x, s) \rightarrow \mathfrak{A}_{8}(x, s):=\int_{\bar{\Omega}^{c}} \partial_{s} \Lambda(x-y, t-s) \cdot u(y, s) d y$ and $\mathfrak{A}_{6}(n, x, s) \rightarrow \mathfrak{A}_{9}(x, s):=-\int_{\Omega^{c}} \Lambda(x-y, t-s) \cdot f(y, s) d y$, as well as $\mathfrak{A}_{7}(n, x, s) \rightarrow$ $\mathfrak{A}_{10}(x, s):=\int_{\bar{\Omega}^{c}} \Lambda(x-y, t-s) \cdot u^{\prime}(s)(y) d y$, for $n \rightarrow \infty$. Lemma 5.2 and the choice of the sequence $\left(R_{n}\right)$ lead to the conclusion that $\mathfrak{A}_{3}(n, x, s)+\mathfrak{A}_{4}(n, x, s) \rightarrow 0$ for $n \rightarrow \infty$.

At this point we have shown that $0=\mathfrak{A}_{1}(x, s)+\mathfrak{A}_{2}(x, s)+\sum_{\mu=8}^{10} \mathfrak{A}_{\mu}(x, s)$. We integrate the preceding equation with respect to $s \in(0, t-\epsilon)$ for any $\epsilon \in(0, t)$, and then let $\epsilon$ tend to zero. First consider $\mathfrak{A}_{1}(x, s)$ and $\mathfrak{A}_{2}(x, s)$. Since $u \mid \Omega_{R_{0}} \times\left(0, T_{0}\right) \in$ $L_{l o c}^{1}\left(\left[0, T_{0}\right), W^{2,1}\left(\Omega_{R_{0}}\right)^{3}\right)$ and $\pi \mid \Omega_{R_{0}} \times\left(0, T_{0}\right) \in L_{l o c}^{1}\left(\left[0, T_{0}\right), W^{1,1}\left(\Omega_{R_{0}}\right)\right)$, a standard 
trace theorem yields that the functions $\partial_{x}^{\beta} u_{j} \mid \partial \Omega \times\left(0, T_{0}\right)$ and $\pi \mid \partial \Omega \times\left(0, T_{0}\right)$ belong to $L_{l o c}^{1}\left(\left[0, T_{0}\right), L^{1}(\partial \Omega)\right)$ for $1 \leq j \leq 3, \beta \in \mathbb{N}_{0}^{3}$ with $|\beta| \leq 1$. Thus we may conclude with Lemma 3.9 that the integral $\int_{0}^{t-\epsilon} \sum_{\mu=1}^{2} \mathfrak{A}_{\mu}(x, s) d s$ converges against the sum of the terms in (5.6) involving $\mathfrak{V}^{(\tau)}$, but with inverse sign, when $\epsilon$ tends to zero. By applying Lemma 5.1 to $u^{(k)}$ for $1 \leq k \leq k_{0}$ and by taking the sum with respect to $k$, we get $\int_{0}^{t-\epsilon}\left(\mathfrak{A}_{8}(x, s)+\mathfrak{A}_{10}(x, s)\right) d s=\mathfrak{B}_{1}(\epsilon, x)+\mathfrak{B}_{2}(\epsilon, x)-\mathfrak{I}^{(\tau)}(u(0))(x, t)$ for $\epsilon \in(0, t)$, with $\mathfrak{B}_{1}(\epsilon, x):=\int_{\bar{\Omega}^{c}} \mathfrak{H}\left(x-y-\tau \epsilon e_{1}, \epsilon\right) u(y, t-\epsilon) d y$ and $\mathfrak{B}_{2}(\epsilon, x)$ defined as the integral $\int_{\bar{\Omega}^{c}} \widetilde{\Lambda}(x-y, \epsilon) \cdot u(y, t-\epsilon) d y$, with $\widetilde{\Lambda}$ from (3.3). Lemma 3.6 yields that $\mathfrak{B}_{2}(\epsilon, x)=$ $\int_{\partial \Omega} A\left(x-y-\tau \epsilon e_{1}, \epsilon\right)\left(n^{(\Omega)}(y) \cdot u(y, t-\epsilon)\right) d o_{y}$ for $\epsilon \in(0, t)$ with $t-\epsilon \in(0, t) \backslash \mathfrak{T}_{T_{0}}$, where $A$ was introduced in (3.4). For $q \in\left(1, \min \left\{\varrho_{k}: 1 \leq k \leq k_{0}\right\}\right]$ and for $R \in\left[S_{0}, \infty\right)$, the function $u \mid \Omega_{R} \times\left[0, T_{0}\right)$ belongs to $C^{0}\left(\left[0, T_{0}\right), L^{q}\left(\Omega_{R}\right)^{3}\right)$. Thus by Theorem 5.1 and the choice of $t$ we get that $\mathfrak{B}_{2}(\epsilon, x) \rightarrow \int_{\partial \Omega}(\nabla \mathfrak{N})(x-y)\left(n^{(\Omega)}(y) \cdot u(y, t)\right) d o_{y}$ for $\epsilon \rightarrow 0$, with the constraint $t-\epsilon \in(0, t) \backslash \mathfrak{T}_{T_{0}}$. Recall that $x$ was taken arbitrarily in $\bar{\Omega}^{c}$.

Choose a sequence $\left(\epsilon_{m}\right)$ in $(0, t)$ with $t-\epsilon_{m} \in(0, t) \backslash \mathfrak{T}_{T_{0}}$ for $m \in \mathbb{N}$ and $\epsilon_{m} \rightarrow 0$. Then by Theorem 3.3 there is a sequence $\left(\epsilon_{m}^{\prime}\right)$ of $\left(\epsilon_{m}\right)$ and a set $N_{t, 1} \subset \bar{\Omega}^{c}$ of measure zero such that $\mathfrak{B}_{1}\left(\epsilon_{m}^{\prime}, x\right) \rightarrow u(x, t)(m \rightarrow \infty)$ for $x \in \bar{\Omega}^{c} \backslash N_{t, 1}$. Moreover Corollary 3.7 implies there is a set $N_{t, 2} \subset \mathbb{R}^{3}$ of measure zero with $\int_{0}^{t}\left|\int_{\bar{\Omega}^{c}} \Lambda(x-y, t-s) \cdot f^{(j)}(y, s) d y\right| d s<\infty$ for $x \in \mathbb{R}^{3} \backslash N_{t, 2}, \quad 1 \leq j \leq n_{0}$. Therefore $\int_{0}^{t-\epsilon_{m}^{\prime}} \mathfrak{A}_{9}(x, s) d s \rightarrow-\mathfrak{R}^{(\tau)}(f)(x, t)(m \rightarrow \infty)$ for $x \in \mathbb{R}^{3} \backslash N_{t, 2}$. At this point we may conclude that for $x \in \bar{\Omega}^{c} \backslash\left(N_{t, 1} \cup N_{t, 2}\right)$, the integral $\int_{0}^{t-\epsilon_{m}^{\prime}}\left(\mathfrak{A}_{1}(x, s)+\mathfrak{A}_{2}(x, s)+\sum_{\mu=8}^{10} \mathfrak{A}_{\mu}(x, s)\right) d s$ converges to $u(x, t)$ minus the right-hand side of (5.4) for $m \rightarrow \infty$. This proves Theorem 5.2.

We may now derive a representation formula which does not contain a pressure term. This formula generalizes [15, Theorem 4.3], where the Stokes system with homogeneous Dirichlet boundary conditions was considered, under stronger assumptions on the data. Recall that at the beginning of this section, we defined $R_{1}:=\left(R_{0}+S_{0}\right) / 2$.

Corollary 5.1 Consider the same situation as in Theorem 5.2. (This means in particular that $u \mid \Omega_{R_{1}} \times\left[0, T_{0}\right) \in C^{0}\left(\left[0, T_{0}\right), L^{q}\left(\Omega_{R_{1}}\right)^{3}\right)$.) Let $t \in\left(0, T_{0}\right) \backslash \mathfrak{T}_{T_{0}}$. Then

$$
\begin{aligned}
& u(x, t)=\mathfrak{R}^{(\tau)}(f)(x, t)+\mathfrak{I}^{(\tau)}(u(0))(x, t)-\sum_{l=1}^{3} \partial x_{l} \mathfrak{V}^{(\tau)}\left(n_{l}^{(\Omega)} u\right)(x, t) \\
& -\int_{\partial \Omega}(\nabla \mathfrak{N})(x-y)\left(n^{(\Omega)}(y) \cdot u(y, t)\right) d o_{y}+\mathfrak{K}_{R_{0}, S_{0}, \varphi_{0}, \Omega, T_{0}}(u)(x, t) \\
& -\int_{\Omega_{R_{1}}} \mathfrak{G}_{R_{0}, S_{0}, \varphi_{0}}(x, y, t) \cdot u(y, 0) d y-\int_{0}^{t} \int_{\Omega_{R_{1}}} \mathfrak{G}_{R_{0}, S_{0}, \varphi_{0}}(x, y, t-s) \cdot f(y, s) d y d s
\end{aligned}
$$

for $x \in{\overline{B_{R_{0}}}}^{c} \backslash N_{t}$, with $N_{t}$ introduced in Theorem 5.2, $\mathfrak{K}_{R_{0}, S_{0}, \varphi_{0}, \Omega, T_{0}}(u)$ defined in (4.17) and $\mathfrak{G}_{R_{0}, S_{0}, \varphi_{0}}$ in (3.13).

Proof: Put $\mathfrak{G}:=\mathfrak{G}_{R_{0}, S_{0}, \varphi_{0}}$. Let $x \in B_{R_{0}}^{c} \backslash N_{t}$ and $j \in\{1,2,3\}$. We transform the term $V(x, t):=\mathfrak{V}_{j}^{(\tau)}\left(\tau n_{1}^{(\Omega)} u+\sum_{l=1}^{3} n_{l}^{(\Omega)}\left(-\partial y_{l} u+\pi e_{l}\right)\right)(x, t)$. Put $q:=\min \left\{\varrho_{k}: 1 \leq\right.$ $\left.k \leq k_{0}\right\}$. Obviously the function $u \mid \Omega_{R_{1}} \times\left(0, T_{0}\right)$ belongs to $W_{l o c}^{1,1}\left(\left[0, T_{0}\right), L^{q}\left(\Omega_{R_{1}}\right)^{3}\right)$ and to $C^{0}\left(\left[0, T_{0}\right), L^{q}\left(\Omega_{R_{1}}\right)^{3}\right)$. By the properties of $\mathfrak{G}$ listed in Lemma 3.11 , by $(5.4)$, the 
relations $\Omega \subset B_{S_{0}}, S_{0}<R_{1}<R_{0}$ and $x \in B_{R_{0}}^{c}$, and by the Divergence theorem, we get

$$
V(x, t)=\sum_{\mu=1}^{3} \mathfrak{A}_{\mu}, \text { with } \quad \mathfrak{A}_{\mu}:=\int_{0}^{t} \int_{\Omega_{R_{1}}} \sum_{k=1}^{3} K_{k}^{(\mu)}(y, s) w_{k}^{(\mu)}(y, s) d y d s \quad(1 \leq \mu \leq 4),
$$

where $K_{k}^{(1)}(y, s):=\nabla_{y} \mathfrak{G}_{j k}(x, y, t-s), w_{k}^{(1)}(y, s):=\nabla_{y} u_{k}(y, s)$, and moreover $K_{k}^{(2)}(y, s):=$ $\partial y_{1} \mathfrak{G}_{j k}(x, y, t-s), w_{k}^{(2)}(y, s):=-\tau u_{k}(y, s)$, and in addition $K_{k}^{(3)}(y, s):=K_{k}^{(4)}(y, s):=$ $\mathfrak{G}_{j k}(x, y, t-s), w_{k}^{(3)}(y, s):=u_{k}^{\prime}(s)(y), w_{k}^{(4)}(y, s):=-f_{k}(y, s)$ for $1 \leq k \leq 3, y \in$ $\Omega_{R_{1}}, s \in(0, t)$. In $\mathfrak{A}_{3}$, we perform an integration by parts with respect to the time variable. To this end, recall that the function $g(r):=\mathfrak{G}(x, \cdot, r) \mid \Omega_{R_{1}}(r \in[0, \infty))$ belongs to $C^{1}\left([0, \infty), L^{q^{\prime}}\left(\Omega_{R_{1}}\right)^{3 \times 3}\right)$ (Lemma 3.12). Thus, by setting $F(r)(V):=\int_{\Omega_{R_{1}}} \mathfrak{G}(x, y, r)$. $V(y) d y$ for $V \in L^{q}\left(\Omega_{R_{1}}\right)^{3}, r \in[0, \infty)$, we obtain a mapping $F \in C^{1}\left([0, \infty),\left[L^{q}\left(\Omega_{R_{1}}\right)^{3}\right]^{\prime}\right)$, with $F^{\prime}(r)(V):=\int_{\Omega_{R_{1}}} \partial_{r} \mathfrak{G}(x, y, r) \cdot V(y) d y$ for $V, r$ as before, where $\partial_{r} \mathfrak{G}(x, y, r)$ is defined in Lemma $3.12\left(y \in \Omega_{R_{1}}, r \in[0, \infty)\right)$. Therefore, due to the regularity properties of $u \mid \Omega_{R_{1}} \times\left(0, T_{0}\right)$ stated above, Lemma 2.5 yields

$$
\begin{aligned}
\mathfrak{A}_{3}= & \sum_{k=1}^{3}\left(\int_{\Omega_{R_{1}}} \mathfrak{G}_{j k}(x, y, 0) u_{k}(y, t) d y-\int_{\Omega_{R_{1}}} \mathfrak{G}_{j k}(x, y, t) u_{k}(y, 0) d y\right. \\
& \left.-\int_{0}^{t} \int_{\Omega_{R_{1}}} \partial_{s} \mathfrak{G}_{j k}(x, y, t-s) u_{k}(y, s) d y d s\right) .
\end{aligned}
$$

The corollary follows with (5.8) and (4.17).

As a last step in this section, we are going to show that equation (5.7) holds under weaker regularity assumption on $u$ near $t=0$. To this end, we establish the following technical point.

Lemma 5.3 Let $k_{0} \in \mathbb{N}, \varrho_{k} \in(1, \infty), u^{(k)} \in C^{0}\left(\left[0, T_{0}\right), L^{\varrho_{k}}\left(\bar{\Omega}^{c}\right)^{3}\right)$ for $1 \leq k \leq k_{0}$, and put $u=\sum_{k=1}^{k_{0}} u^{(k)}$. In particular $u \mid \Omega_{R_{1}} \times\left[0, T_{0}\right) \in C^{0}\left(\left[0, T_{0}\right), L^{q}\left(\Omega_{R_{1}}\right)^{3}\right)$ for $q \in$ $(1, \infty)$ with $q \leq \varrho_{k}$ for $1 \leq k \leq k_{0}$. Suppose in addition that $u(s) \in W_{l o c}^{1,1}\left(\bar{\Omega}^{c}\right)^{3}$ for a. e. $s \in\left(0, T_{0}\right)$ and $\nabla_{x} u \mid \Omega_{R_{1}} \times\left(0, T_{0}\right) \in L_{l o c}^{1}\left(\left[0, T_{0}\right), L^{q_{1}}\left(\Omega_{R_{1}}\right)^{9}\right)$ for some $q_{1} \in(1, \infty)$. Furthermore let $n_{0} \in \mathbb{N}, p_{j} \in(1, \infty), f^{(j)} \in L_{\text {loc }}^{1}\left(\left[0, T_{0}\right), L^{p_{j}}\left(\bar{\Omega}^{c}\right)^{3}\right)$ for $1 \leq j \leq n_{0}$, and put $f:=\sum_{j=1}^{n_{0}} f^{(j)}$.

Choose functions $\zeta_{\epsilon}$ for $\epsilon \in(0, \infty)$ as in the passage preceding Lemma 3.14, and put $u_{\epsilon}^{(k)}(s):=\zeta_{\epsilon}(s) u^{(k)}(s), u_{\epsilon}(s):=\zeta_{\epsilon}(s) u(s), f_{\epsilon}(s):=\zeta_{\epsilon}(s) f(s)$ and $g_{\epsilon}(s):=f_{\epsilon}(s)+$ $\zeta_{\epsilon}^{\prime}(s) u(s)$ for $s \in\left(0, T_{0}\right), \epsilon \in(0, \infty), 1 \leq k \leq k_{0}$.

Let $t \in\left(0, T_{0}\right)$ with $u(t) \mid \Omega_{R_{1}} \in W^{1,1}\left(\Omega_{R_{1}}\right)^{3}$ and $\operatorname{div}_{x}\left(u(t) \mid \Omega_{R_{1}}\right)=0$. Suppose there is some $\epsilon_{0} \in(0, \infty)$ such that for $\epsilon \in\left(0, \epsilon_{0}\right]$, equation (5.7) holds with $u, f$ replaced by $u_{\epsilon}$ and $g_{\epsilon}$, respectively, if $x \in \overline{B_{R_{0}}} \backslash \backslash N_{t, \epsilon}$ for some zero-measure subset $N_{t, \epsilon}$ of $\overline{B_{R_{0}}}$ (This means in particular that the second from last term on the right-hand side of (5.7) vanishes.) Then there is some zero-measure set $N_{t} \subset \overline{B_{R_{0}}}$ c such that equation (5.7) remains valid for $u$ and $f$ if $x \in{\overline{B_{R_{0}}}}^{c} \backslash N_{t}$.

Proof: Put $f_{\epsilon}^{(j)}(s):=\zeta_{\epsilon}(s) f^{(j)}(s)$ for $s \in\left(0, T_{0}\right), 1 \leq j \leq n_{0}$. We note that $u_{\epsilon}=$ $\sum_{k=1}^{k_{0}} u_{\epsilon}^{(k)}, f_{\epsilon}=\sum_{j=1}^{n_{0}} f_{\epsilon}^{(j)},\left(g_{\epsilon}-f_{\epsilon}\right)(s)=\zeta_{\epsilon}^{\prime}(s) u(s)$ for $s \in\left(0, T_{0}\right)$, the functions $u_{\epsilon}^{(k)}, u_{\epsilon}$ 
and $f_{\epsilon}^{(j)}$ have the same regularity properties as $u^{(k)}, u$ and $f^{(j)}$, respectively, for $1 \leq k \leq$ $k_{0}, 1 \leq j \leq n_{0}, \epsilon \in(0, \infty)$.

Put $q:=\min \left(\left\{\varrho_{k}: 1 \leq k \leq k_{0}\right\} \cup\left\{q_{1}\right\}\right)$. Obviously the functions $u \mid \Omega_{R_{1}} \times\left(0, T_{0}\right)$ and $\partial x_{k} u \mid \Omega_{R_{1}} \times\left(0, T_{0}\right)$ belong to $L_{\text {loc }}^{1}\left(\left[0, T_{0}\right), L^{q}\left(\Omega_{R_{1}}\right)^{3}\right)(1 \leq k \leq 3)$. For $\beta \in \mathbb{N}_{0}^{3}$ with $|\beta| \leq$ $1, \epsilon \in(0, \infty)$ and a. e. $s \in\left(0, T_{0}\right),\left\|\partial_{y}^{\beta}\left(u_{\epsilon}-u\right)(s)\left|\Omega_{R_{1}}\left\|_{q}=\left(1-\zeta_{\epsilon}(s)\right)\right\| \partial_{y}^{\beta} u(s)\right| \Omega_{R_{1}}\right\|_{q} \leq$ $\left\|\partial_{y}^{\beta} u(s) \mid \Omega_{R_{1}}\right\|_{q}$, and similarly $\left\|\left(f_{\epsilon}^{(j)}-f^{(j)}\right)(s)\right\|_{p_{j}} \leq\left\|f^{(j)}(s)\right\|_{p_{j}}\left(1 \leq j \leq n_{0}\right)$. Hence by Lebesgue's theorem,

$$
\left\|\partial_{y}^{\beta}\left(u_{\epsilon}-u\right) \mid \Omega_{R_{1}} \times(0, t)\right\|_{q, 1 ; t} \rightarrow 0 \text { and }\left\|\left(f_{\epsilon}^{(j)}-f^{(j)}\right) \mid \bar{\Omega}^{c} \times(0, t)\right\|_{p_{j}, 1 ; t} \rightarrow 0(\epsilon \downarrow 0)
$$

for $1 \leq j \leq n_{0}$ and for $\beta$ as before. By the choice of $\zeta_{\epsilon}$,

$$
\left\|\left(u_{\epsilon}-u\right)(t) \mid \Omega_{R_{1}}\right\|_{q}=0 \text { for } \epsilon \in(0, t / 2] .
$$

Let $x \in B_{R_{0}}^{c}$, Put $\mathfrak{G}:=\mathfrak{G}_{R_{1}, S_{0}, \varphi_{0}}$ (see (3.13)). Theorem 4.2 and (5.9) imply that $\int_{0}^{t} \int_{\Omega_{R_{1}}} \partial_{s}^{1-\sigma} \partial y_{l}^{\sigma} \mathfrak{G}(x, y, t-s) \cdot\left(\partial_{l}^{\mu} u_{\epsilon}-\partial_{l}^{\mu} u\right)(y, s) d y d s \rightarrow 0(\epsilon \downarrow 0)$ for $\mu, \sigma \in\{0,1\}, l \in$ $\{1,2,3\}$, and $\int_{0}^{t} \int_{\Omega_{R_{1}}} \mathfrak{G}(x, y, t-s) \cdot\left(f_{\epsilon}^{(j)}-f^{(j)}\right)(y, s) d y d s \rightarrow 0(\epsilon \downarrow 0)$ for $1 \leq j \leq n_{0}$. Obviously by $(5.10) \int_{\Omega_{R_{1}}} \mathfrak{G}(x, y, 0) \cdot\left(u_{\epsilon}-u\right)(y, t) d y=0$ for $\epsilon \in(0, t / 2]$.

The preceding observations and (5.10) mean that $\left|\mathfrak{K}_{R_{0}, S_{0}, \varphi_{0}, \Omega, T_{0}}\left(u_{\epsilon}-u\right)(x, t)\right| \rightarrow 0$ for $\epsilon \downarrow 0$. Moreover, by Lemma 4.3, the term $\mathfrak{A}_{\epsilon}:=\left|\sum_{l=1}^{3} \partial x_{l} \mathfrak{V}^{(\tau)}\left(n_{l}^{(\Omega)}\left(u_{\epsilon}-u\right)\right)(x, t)\right|$ is bounded by $\mathfrak{C}\left(\left\|u_{\epsilon}-u\left|\Omega_{R_{1}} \times(0, t)\left\|_{q, 1 ; t}+\right\| \nabla_{y}\left(u_{\epsilon}-u\right)\right| \Omega_{R_{1}} \times(0, t)\right\|_{q, 1 ; t}\right)$ for $\epsilon \in(0, \infty)$. Thus $\mathfrak{A}_{\epsilon} \rightarrow 0$ for $\epsilon \downarrow 0$ by (5.9). Since $x \in B_{R_{0}}^{c}, \Omega \subset B_{S_{0}}$ and by the choice of $t$, Lemma 4.4 yields that $\mathfrak{E}_{\epsilon}:=\left|\int_{\partial \Omega}(\nabla \mathfrak{N})(x-y)\left(n^{(\Omega)}(y) \cdot\left(u_{\epsilon}-u\right)(y, t)\right) d o_{y}\right| \leq \mathfrak{C}\left\|\left(u_{\epsilon}-u\right)(t) \mid \Omega_{R_{1}}\right\|_{q}$, so $\mathfrak{E}_{\epsilon} \rightarrow 0(\epsilon \downarrow 0)$ by (5.10). Next we apply Lemma 3.14 with $u \mid \Omega_{R_{1}} \times\left[0, T_{0}\right)$ in the role of $v$. We obtain that $\int_{0}^{t} \int_{\Omega_{R_{1}}} \mathfrak{G}(x, y, t-s) \cdot \zeta_{\epsilon}^{\prime}(s) u_{\epsilon}(y, s) d y d s \rightarrow \int_{\Omega_{R_{1}}} \mathfrak{G}(x, y, t) \cdot u(y, 0) d y$ for $\epsilon \downarrow 0$. Moreover, using Lemma 3.15 with $q, u$ replaced by $\varrho_{k}$ and $u^{(k)}$, respectively, we get $\mathfrak{R}^{(\tau)}\left(h_{\epsilon}^{(k)}\right)(x, t) \rightarrow \mathfrak{I}^{(\tau)}\left(u^{(k)}(0)\right)(x, t)(\epsilon \downarrow 0)$ for $1 \leq k \leq k_{0}$, where $h_{\epsilon}^{(k)}(s):=$ $\zeta_{\epsilon}^{\prime}(s) u^{(k)}(s)$ for $s \in\left[0, T_{0}\right), \epsilon>0$. Therefore $\mathfrak{R}^{(\tau)}\left(h_{\epsilon}\right)(x, t) \rightarrow \mathfrak{I}^{(\tau)}(u(0))(\epsilon \downarrow 0)$, with $h_{\epsilon}(s):=\zeta_{\epsilon}^{\prime}(s) u(s)$ for $s, \epsilon$ as before.

Up to this point, the vector $x$ was arbitrary but fixed in $B_{R_{0}}^{c}$. We still have to consider $\mathfrak{R}^{(\tau)}\left(f_{\epsilon}\right)$. The second relation in (5.9) and Corollary 3.7 yield that $\left\|\mathfrak{R}^{(\tau)}\left(f_{\epsilon}^{(j)}-f^{(j)}\right)(t)\right\|_{p_{j}} \rightarrow$ $0(\epsilon \downarrow 0)$ for $1 \leq j \leq n_{0}$. This implies there is a sequence $\left(\epsilon_{n}\right)$ in $(0, \infty)$ with $\epsilon_{n} \rightarrow 0$ and $\mathfrak{R}^{(\tau)}\left(f_{\epsilon}-f\right)(x, t) \rightarrow 0(n \rightarrow \infty)$ for a. e. $x \in \mathbb{R}^{3}$. The preceding convergence results taken together yield Lemma 5.3.

Now we are able to establish (5.7) for solutions of the Oseen system that are less regular near $t=0$ than those considered in Corollary 5.1, except that we additionally suppose that $\nabla_{y} u \mid \Omega_{R_{0}} \times\left(0, T_{0}\right) \in L_{l o c}^{1}\left(\left[0, T_{0}\right), L^{q_{1}}\left(\Omega_{R_{0}}\right)^{9}\right)$.

Corollary 5.2 Let $k_{0} \in \mathbb{N}, \varrho_{k} \in(1, \infty)$, and let $u^{(k)}$ belong to $C^{0}\left(\left[0, T_{0}\right), L^{\varrho_{k}}\left(\bar{\Omega}^{c}\right)^{3}\right)$ and to $W_{l o c}^{1,1}\left(0, T_{0}, L^{\varrho_{k}}\left(\bar{\Omega}^{c}\right)^{3}\right)$, for $1 \leq k \leq k_{0}$. Put $u=\sum_{k=1}^{k_{0}} u^{(k)}$. Note that $u \mid \Omega_{R_{1}} \times$ $\left[0, T_{0}\right) \in C^{0}\left(\left[0, T_{0}\right), L^{q}\left(\Omega_{R_{1}}\right)^{3}\right)$ for $q \in\left(1, \min \left\{\varrho_{k}: 1 \leq k \leq k_{0}\right\}\right]$. Let $\pi:\left(0, T_{0}\right) \mapsto$ $W_{\text {loc }}^{1,1}\left(\bar{\Omega}^{c}\right), n_{0} \in \mathbb{N}, p_{j} \in(1, \infty), f^{(j)} \in L_{\text {loc }}^{1}\left(0, T_{0}, L^{p_{j}}\left(\bar{\Omega}^{c}\right)^{3}\right)$ for $1 \leq j \leq n_{0}$. Suppose that $u(s) \in W_{l o c}^{2,1}\left(\bar{\Omega}^{c}\right)^{3}$ and (5.4) holds for a. e. $s \in\left(0, T_{0}\right)$, with $f=\sum_{j=1}^{n_{0}} f^{(j)}, u^{\prime}=$ $\sum_{k=1}^{k_{0}}\left(u^{(k)}\right)^{\prime}$. 
In addition suppose there is $q_{1} \in(1, \infty)$ such that $\nabla_{y} u(s) \mid{\overline{B_{R_{0}}}}^{c} \in L^{q_{1}}\left(\overline{B_{R_{0}}}\right)^{9}$ for a. e. $s \in\left(0, T_{0}\right)$ and $\nabla_{y} u \mid \Omega_{R_{0}} \times\left(0, T_{0}\right) \in L_{l o c}^{1}\left(\left[0, T_{0}\right), L^{q_{1}}\left(\Omega_{R_{0}}\right)^{9}\right)$. Further assume that $u \mid \Omega_{R_{0}} \times$ $\left(0, T_{0}\right) \in L_{l o c}^{1}\left(0, T_{0}, W^{2,1}\left(\Omega_{R_{0}}\right)^{3}\right), \pi \mid \Omega_{R_{0}} \times\left(0, T_{0}\right) \in L_{l o c}^{1}\left(0, T_{0}, W^{1,1}\left(\Omega_{R_{0}}\right)\right)$, and there are numbers $m_{0} \in \mathbb{N}, \gamma_{j} \in(1, \infty)$ as well as functions $\pi^{(j)}:\left(0, T_{0}\right) \mapsto L_{\text {loc }}\left(\overline{B_{R_{0}}}{ }^{c}\right)$ for $1 \leq j \leq$ $m_{0}$ such that (5.5) is valid for a. e. $s \in\left(0, T_{0}\right)$. Choose a set $\mathfrak{T}_{T_{0}}$ as in Theorem 5.2, that is, $\mathfrak{T}_{T_{0}}$ is a zero-measure subset of $\left(0, T_{0}\right)$ such that $u(t) \mid \Omega_{R} \in W^{1,1}\left(\Omega_{R}\right)^{3}\left(R \in\left[S_{0}, \infty\right)\right)$ and $\operatorname{div}_{x} u(t)=0$ for $t \in\left(0, T_{0}\right) \backslash \mathfrak{T}_{T_{0}}$.

Let $t \in\left(0, T_{0}\right) \backslash \mathfrak{T}_{T_{0}}$. Then there is a zero-measure set $N_{t} \subset{\overline{B_{R_{0}}}}^{c}$ such that (5.7) holds for $x \in{\overline{B_{R_{0}}}}^{c} \backslash N_{t}$.

Proof: For $\epsilon \in(0, \infty)$, choose $\zeta_{\epsilon}$ as in the passage preceding Lemma 3.14, and define $u_{\epsilon}^{(k)}, u_{\epsilon}$ and $f_{\epsilon}^{(j)}\left(1 \leq k \leq k_{0}, 1 \leq j \leq n_{0}\right)$ as in Lemma 5.3. Further put $\pi_{\epsilon}(s):=\zeta_{\epsilon}(s) \pi(s), \pi_{\epsilon}^{(j)}(s):=\zeta_{\epsilon}(s) \pi^{(j)}(s)$ for $s \in\left(0, T_{0}\right), 1 \leq j \leq m_{0}$. Since all functions with index $\epsilon$ vanish on $(0, \epsilon)$, all assumptions in Theorem 5.2 except the system in (5.4) (momentum equation) are fulfilled if the functions in that theorem are replaced by the corresponding functions with the index $\epsilon$, for arbitrary but fixed $\epsilon \in(0, \infty)$. In order to state the variant of (5.4) which is valid for the functions with index $\epsilon$, put $p_{n_{0}+k}:=\varrho_{k}, f_{\epsilon}^{n_{0}+k}(s):=\zeta_{\epsilon}^{\prime}(s) u^{(k)}(s)$ for $s \in\left(0, T_{0}\right), \epsilon \in(0, \infty), 1 \leq k \leq k_{0}$. Then $f_{\epsilon}^{(j)} \in L_{l o c}^{1}\left(\left[0, T_{0}\right), L^{p_{j}}\left(\bar{\Omega}^{c}\right)^{3}\right)$ for $\epsilon>0,1 \leq j \leq k_{0}+n_{0}$, and the system in (5.4) is valid with $u, \pi, f$ replaced by $u_{\epsilon}, \pi_{\epsilon}$ and $\sum_{j=1}^{n_{0}+k_{0}} f_{\epsilon}^{(j)}$, respectively. At this point we may apply Corollary 5.1, obtaining that for $\epsilon>0$, there is a zero-measure set $N_{\epsilon, t} \subset{\overline{B_{R_{0}}}}^{c}$ such that equation (5.7) holds for $x \in \overline{B_{R_{0}}} \backslash \backslash N_{\epsilon, t}$ with the same replacements for $u$ and $f$. As in Lemma 5.3, define $g_{\epsilon}(s):=\sum_{j=1}^{n_{0}} f_{\epsilon}^{(j)}(s)+\zeta_{\epsilon}^{\prime}(s) u(s)\left(s \in\left(0, T_{0}\right), \epsilon>0\right)$. Obviously $g_{\epsilon}=\sum_{j=1}^{n_{0}+k_{0}} f_{\epsilon}^{(j)}(\epsilon \in(0, \infty))$, so we now get that equation (5.7) is verified with $u_{\epsilon}$ and $g_{\epsilon}$ in the role of $u$ and $f$, respectively, for $x \in{\overline{B_{R_{0}}}}^{c} \backslash N_{\epsilon, t}$. In view of the assumption $\nabla_{y} u \mid \Omega_{R_{0}} \times\left(0, T_{0}\right) \in L_{l o c}^{1}\left(\left[0, T_{0}\right), L^{q_{1}}\left(\Omega_{R_{0}}\right)^{9}\right)$, we see that all the conditions in Lemma 5.3 are fulfilled. Therefore this lemma yields the claim of Corollary 5.2.

\section{Decay estimates of solutions to (1.1).}

By combining the representation formula (5.7) with the estimates in Section 4, we may now derive our result on the decay behaviour of solutions to (1.1). The term $1 /\left(2 \sigma_{j}\right)(1 \leq$ $j \leq 3)$ in the exponent of the decay factor $|x| \nu(x)$ in (6.11) below constitutes a link between $L^{p}$-integrability in time of $u$ and pointwise spatial decay of $u$. This is the link mentioned in the title of this work and in Section 1.

Theorem 6.1 Consider the situation in Corollary 5.2. Choose some number $q \in(1, \infty)$ with $q \leq \min \left\{\varrho_{k}: 1 \leq k \leq k_{0}\right\}$, and put $Z_{R_{1}, T_{0}}:=\Omega_{R_{1}} \times\left(0, T_{0}\right)$. Suppose in addition that $u \mid Z_{R_{1}, T_{0}} \in L^{\infty}\left(0, T_{0}, L^{q}\left(\Omega_{R_{1}}\right)^{3}\right)$, and that there are numbers $\sigma_{1}, \sigma_{2}, \sigma_{3} \in[1, \infty]$ with $u\left|Z_{R_{1}, T_{0}} \in L^{\sigma_{1}}\left(0, T_{0}, L^{q}\left(\Omega_{R_{1}}\right)^{3}\right), \quad \nabla_{x} u\right| Z_{R_{1}, T_{0}} \in L^{\sigma_{2}}\left(0, T_{0}, L^{q}\left(\Omega_{R_{1}}\right)^{9}\right)$ and $f \mid Z_{R_{1}, T_{0}} \in$ $L^{\sigma_{3}}\left(0, T_{0}, L^{q}\left(\Omega_{R_{1}}\right)^{3}\right)$. Put

$$
\mathfrak{F}(u, f):=\left\|u\left|Z_{R_{1}, T_{0}}\left\|_{q, \sigma_{1} ; T_{0}}+\right\| u\right| Z_{R_{1}, T_{0}}\right\|_{q, \infty ; T_{0}}+\left\|\nabla u\left|Z_{R_{1}, T_{0}}\left\|_{q, \sigma_{2} ; T_{0}}+\right\| f\right| Z_{R_{1}, T_{0}}\right\|_{q, \sigma_{3} ; T_{0}} .
$$


Then there is a zero-measure subset $\mathfrak{S}_{T_{0}}$ of $\left(0, T_{0}\right)$ such that

$$
\begin{aligned}
& \left|\left[\partial_{x}^{\alpha} u-\partial_{x}^{\alpha} \mathfrak{R}^{(\tau)}(f)-\partial_{x}^{\alpha} \mathfrak{I}^{(\tau)}(u(0))\right](x, t)\right| \\
& \leq \mathfrak{C} \mathfrak{F}(u, f)\left[(|x| \nu(x))^{-(3+|\alpha|) / 2+1 /\left(2 \min \left\{\sigma_{1}^{\prime}, \sigma_{2}^{\prime}, \sigma_{3}^{\prime}\right\}\right)}+|x|^{-\gamma-|\alpha|}\right]
\end{aligned}
$$

for $t \in\left(0, T_{0}\right) \backslash \mathfrak{S}_{T_{0}}, x \in{\overline{B_{R_{0}}}}^{c} \backslash N_{t}$ with some zero-measure set $N_{t} \subset \overline{B_{R_{0}}}$, and for $\alpha \in \mathbb{N}_{0}^{3}$ with $|\alpha| \leq 1$, where $\gamma=3$ if $\int_{\partial \Omega} u(t) \cdot n^{(\Omega)} d o_{x}=0$ for $t \in\left(0, T_{0}\right)$, and $\gamma=2$ else.

In particular, if $\gamma=3$, the following inequality holds for $t, x, \alpha$ as before:

$$
\begin{aligned}
& \left|\left[\partial_{x}^{\alpha} u-\partial_{x}^{\alpha} \mathfrak{R}^{(\tau)}(f)-\partial_{x}^{\alpha} \mathfrak{I}^{(\tau)}(u(0))\right](x, t)\right| \\
& \leq \mathfrak{C} \mathfrak{F}(u, f)(|x| \nu(x))^{-(3+|\alpha|) / 2+1 /\left(2 \min \left\{\sigma_{1}^{\prime}, \sigma_{2}^{\prime}, \sigma_{3}^{\prime}\right\}\right)} .
\end{aligned}
$$

Proof: We have $u \mid Z_{R_{1}, T_{0}} \in C^{0}\left(\left[0, T_{0}\right), L^{q}\left(\Omega_{R_{1}}\right)^{9}\right)$, so $\left\|u(t)\left|\Omega_{R_{1}}\left\|_{q} \leq\right\| u\right| Z_{R_{1}, T_{0}}\right\|_{q, \infty ; T_{0}}$ for any $t \in\left[0, T_{0}\right)$, without any exceptional values of $t$. Therefore inequality (6.11) follows from (5.7) on the one hand (see Corollary 5.2), and Theorem 4.2, Corollary 4.2, 4.3, Lemma 4.3 and 4.4 on the other. If $\gamma=3$, the term $|x|^{-\gamma-|\alpha|}$ in (6.11) is bounded by $\mathfrak{C}(|x| \nu(x))^{-(3+|\alpha|) / 2}$ because $|x| \geq C(R) \nu(x)$ for $x \in B_{R}^{c}, \quad R \in(0, \infty)$. Therefore, in the case $\gamma=3$, inequality (6.12) is an immediate consequence of (6.11). Note that $\mathfrak{R}^{(\tau)}(f)(t) \in W^{1,1}\left(\mathbb{R}^{3}\right)^{3}$ only for a. e. $t \in(0, \infty)$ (Lemma 3.8), so the set $\mathfrak{S}_{T_{0}}$ may be larger than the set $\mathfrak{T}_{T_{0}}$ in Corollary 5.2.

A remark is perhaps in order with respect to the terms $\partial_{x}^{\alpha} \mathfrak{R}^{(\tau)}(f)$ and $\mathfrak{I}^{(\tau)}(u(0))$ on the left-hand side of (6.11). In [13, Theorem 3.1], the assumptions on $f$ are chosen in such a way that the term $\left|\partial_{x}^{\alpha} \mathfrak{R}^{(\tau)}(f)(x, t)\right|$ is bounded by $\mathfrak{C}(|x| \nu(x))^{-(2+|\alpha|) / 2}$ for $x \in B_{R}^{c}, t \in$ $\left(0, T_{0}\right), \alpha \in \mathbb{N}_{0}^{3}$ with $|\alpha| \leq 1$, where $R$ is some suitably large positive real. If $f$ is given as in Lemma 4.2 , we even obtain the upper bound $\mathfrak{C}(|x| \nu(x))^{-(3+|\alpha|) / 2}$, which corresponds to the strongest possible decay in the sense that the quantity $\left|\partial_{x}^{\alpha} \Lambda(x, t)\right|$ decreases in just this way (Corollary 3.3). In other words: The decay of $\left|\partial_{x}^{\alpha} \mathfrak{R}^{(\tau)}(f)(x, t)\right|$ in Lemma 4.2 is the same as that of the fundamental solution $\Lambda$ of (1.1). In any case the asymptotics of $\mathfrak{R}^{(\tau)}(f)$ are a direct and exclusive consequence of the assumptions on $f$. The situation is similar with respect to the function $\mathfrak{I}^{(\tau)}(u(0))$, whose behaviour is determined by the initial data $u(0)$. We refer to [12, Theorem 1.1], where the conditions on $u(0)$ lead to the upper bound $\mathfrak{C}(|x| \nu(x))^{-(2+|\alpha|) / 2}$ for $x, t, \alpha$ as before, and to Lemma 4.1, where $u(0)$ is supposed to be integrable and have compact support. In that latter case we get the optimal decay bound $\mathfrak{C}(|x| \nu(x))^{-(3+|\alpha|) / 2}$. These features of the asymptotic behaviour of $\mathfrak{R}^{(\tau)}(f)$ and $\mathfrak{I}^{(\tau)}(u(0))$ explain why inequality (6.11) implies (1.3) under suitable decay properties of $f$ and $u(0)$.

We further indicate that the sum $\partial_{x}^{\alpha} \mathfrak{R}^{(\tau)}(f)+\mathfrak{I}^{(\tau)}(u(0))$ constitutes a solution to (1.1) in the whole space $\mathbb{R}^{3}(\Omega=\emptyset)$, with initial data $u(0)$ if $u(0)$ is solenoidal ([10, Theorem 2.16], Lemma 3.7, Theorem 3.3, (3.3), Corollary 3.5). So left-hand side of inequality (6.11) can be interpreted as the perturbation generated in the fluid by the presence of the rigid body. But it is precisely this perturbation which is of interest. So inequality (6.11) may be seen as a decay estimate of that part of the flow which is relevant here.

We finally present two existence results for solutions to (1.1) which satisfy the assumptions of the preceding theorem. The first - Theorem 6.2 below - deals with $L^{2}$-strong solutions, 
of a type familiar in the context of the Stokes system. Theorem 6.1 may be applied to these solutions with $\sigma_{1}=\sigma_{2}=2$ and $\gamma=3$. The choice $\gamma=3$ is possible because homogeneous Dirichlet boundary conditions are imposed in Theorem 6.2. Inequality (6.12) then yields that its left-hand side is bounded by $\mathfrak{C}(|x| \nu(x))^{-5 / 4-|\alpha| / 2}$. We indicate that the velocity part of solutions to (1.1) constructed in [10] and [13] belongs to the uniqueness class $L_{l o c}^{2}\left([0, \infty), W^{1,2}\left(\bar{\Omega}^{c}\right)^{3}\right)([13$, Corollary 2.28$])$. Thus, if both Theorem 6.2 and the theory in [10] and [13] yield existence of a solution to the initial-boundary value problem considered in Theorem 6.2, these two solutions coincide, so we may compare the decay rate provided by (6.12) with the one exhibited in [10] and [13]. In these latter references, we only obtained the rate $-1-|\alpha| / 2$ ([13, Theorem 2.26, Lemma 3.2]]), vs. $-5 / 4-|\alpha| / 2$ in (6.12), as mentioned above. But this comparison is not fully satisfying because it requires additional assumptions on the data, in the context of both Theorem 6.2 and [10] and [13]. In fact, the conditions on $U_{0}$ in [10] and [13] are weaker than those in Theorem 6.2, and those on $f$ are different. Also, nonhomogeneous Dirichlet boundary data are admitted in [10] and [13]. However, reference [17] generalizes the theory presented here, fully covering the framework in [10] and [13]. In particular, for the situation considered in these latter articles, we again obtain the decay rate $-5 / 4-|\alpha| / 2$ ([17, Theorem $6.1,6.2])$.

Theorem 6.2 Suppose that $\Omega$ is $C^{2}$-bounded. Let $f \in L^{2}\left(0, \infty, L^{2}\left(\bar{\Omega}^{c}\right)^{3}\right)$ and $U_{0} \in$ $W_{0}^{1,2}\left(\bar{\Omega}^{c}\right)^{3}$ with div $U_{0}=0$. Then there is a uniquely determined pair of functions $(u, \pi)$ with the properties to follow:

The function $u$ is in $C^{0}\left([0, \infty), L^{2}\left(\bar{\Omega}^{c}\right)^{3}\right), L^{2}\left(0, \infty, L^{6}\left(\bar{\Omega}^{c}\right)^{3}\right)$ and $L^{\infty}\left(0, \infty, L^{2}\left(\bar{\Omega}^{c}\right)^{3}\right)$, as well as in $W_{\text {loc }}^{1,2}\left([0, \infty), L^{2}\left(\bar{\Omega}^{c}\right)^{3}\right)$ and $L_{\text {loc }}^{2}\left([0, \infty), W^{2,2}\left(\bar{\Omega}^{c}\right)^{3}\right)$. Moreover $\partial x_{k} u$ and $\partial_{t} u$ belong to $L^{2}\left(0, \infty, L^{2}\left(\bar{\Omega}^{c}\right)^{3}\right)$ for $1 \leq k \leq 3$, and $\pi$ to $L^{2}\left(0, \infty, L^{6}\left(\bar{\Omega}^{c}\right)\right)$, with $\pi(t) \in$ $W_{\text {loc }}^{1,2}\left(\bar{\Omega}^{c}\right)$ for $t \in(0, \infty)$ and $\nabla_{x} \pi \in L^{2}\left(0, \infty, L^{2}(\bar{\Omega})^{3}\right)$.

In addition the pair $(u, \pi)$ solves (5.4) as well as the equations $u(0)=U_{0}$ and $u(t) \mid \partial \Omega=0$ for $t \in(0, \infty)$. This pair of functions satisfies the assumptions of Corollary 5.2 and Theorem 6.1 with $n_{0}=k_{0}=m_{0}=1, \gamma_{1}=6, \pi^{(1)}=\pi \mid{\overline{B_{R_{0}}}}^{c}$ and with $\varrho_{1}, p_{1}, q_{1}, q$, and $\sigma_{j}$ for $j \in\{1,2,3\}$ all being equal to 2 .

Proof: By the same arguments as in the case of the Stokes system, it may be shown there is an $L^{2}$-weak solution of (1.1) characterized by the relations $u \in L^{\infty}\left(0, \infty, L^{2}\left(\bar{\Omega}^{c}\right)^{3}\right)$ and $\nabla_{x} u \in L^{2}\left(0, \infty, L^{2}\left(\bar{\Omega}^{c}\right)^{9}\right)$; compare [13, Theorem 2.12] for existence (with a precise statement on a weak form of (1.1)), and [11, Theorem 3.7] for uniqueness. The arguments used in the Stokes case may be found in [44, p. 171-176 and p.180]. The equation $\int_{\bar{\Omega}^{c}} \partial_{1} V \cdot V d x=0$ for $V \in W_{0}^{1,2}\left(\bar{\Omega}^{c}\right)^{3}$ is the reason why the Oseen term does not generate a major problem. In a second step, we consider this function $u$ as the velocity part of a weak solution to the time-dependent Stokes system with right-hand side $f-\tau \partial x_{1} u$. By existence and uniqueness results for this latter system, as presented in [40, Section IV.2], we obtain what is claimed in the theorem. More details and more precise references may be found in [15, Theorem 3.2 and 3.3] and their proof.

The solutions of (1.1) considered in the next theorem are not in general covered by the theory in [10] or [13], whereas Theorem 6.1 yields the upper bound $(|x| \nu(x))^{-(3+|\alpha|) / 2+1 /\left(2 q^{\prime}\right)}$ for the left-hand side of (6.11). 
Theorem 6.3 Suppose that $\Omega$ is $C^{2}$-bounded and $T_{0}<\infty$, and let $q \in(1, \infty)$. Put $Z_{T_{0}}:=\bar{\Omega}^{c} \times\left(0, T_{0}\right)$, and let $W_{q, T_{0}}$ denote the space of all function $u \in L^{q}\left(Z_{T_{0}}\right)^{3}$ such that the weak derivativs $\partial_{t} u, \partial x_{k} u$ and $\partial x_{k} x_{l} u$ exist for $1 \leq k, l \leq 3$ and belong to $L^{q}\left(Z_{T_{0}}\right)^{3}$. Let \|\|$_{q, Z_{T_{0}}}$ denote the corresponding norm of $W_{q, T_{0}}$. Let $J_{q}^{2-2 / q}\left(\bar{\Omega}^{c}\right)$ stand for the closure of $C_{0, \sigma}^{\infty}\left(\bar{\Omega}^{c}\right)$ with respect to the norm \|\| defined by

$$
\|U\|:=\inf \left\{\|u\|_{q, T_{0}}: u \in W_{q, T_{0}}, u(t) \mid \partial \Omega=0 \text { for } t \in\left(0, T_{0}\right), u(0)=U\right\}
$$

for $U \in C_{0, \sigma}^{\infty}\left(\bar{\Omega}^{c}\right)([42, p .487])$.

Take $f \in L^{q}\left(Z_{T_{0}}\right)^{3}$ and $U_{0} \in J_{q}^{2-2 / q}\left(\bar{\Omega}^{c}\right)$. Then there is a uniquely determined pair of functions $(u, \pi)$ with $u \in W_{q, T_{0}}$ and $\pi:\left(0, T_{0}\right) \mapsto W_{\text {loc }}^{1, q}\left(\bar{\Omega}^{c}\right)$ such that $\nabla_{x} \pi$ belongs to $L^{q}\left(Z_{T_{0}}\right)^{3}$, equation (1.1) holds, and the initial condition $u(0)=U_{0}$ as well as the boundary conditions $u(t) \mid \partial \Omega=0$ for $t \in\left(0, T_{0}\right)$ are fulfilled. These functions $u$ and $\pi$ satisfy the assumptions of Corollary 5.2 and Theorem 6.1 with $n_{0}=k_{0}=m_{0}=1, \pi^{(1)}=\pi \mid \overline{B_{R_{0}}} c$ and with $\varrho_{1}, p_{1}, q_{1}, \gamma_{1}, q$, and $\sigma_{j}$ for $j \in\{1,2\}$ all being equal to $q$.

Proof: The results in [42, Theorem 4.2 and p. 513-515] yield all the claims of the theorem except those stated in the last sentence, pertaining to the assumptions of Corollary 5.2 and Theorem 6.1. But these latter claims are an easy conclusion of the part of the theorem taken from [42]. To see this, we make the usual identification of $L^{q}\left(Z_{T_{0}}\right)^{3}$ and $L^{q}\left(0, T_{0}, L^{q}\left(\bar{\Omega}^{c}\right)^{3}\right)$; see Lemma 2.3 and [11, Lemma 2.1] in this respect. Since $u$ and $\partial_{t} u$ belong to $L^{q}\left(Z_{T_{0}}\right)^{3}$, we get by Theorem 2.5 that for $\varphi \in C_{0}^{\infty}\left(\left(0, T_{0}\right)\right)$ and $\psi \in C_{0}^{\infty}\left(\bar{\Omega}^{c}\right)^{3}$, the integrals $\int_{\bar{\Omega}^{c}} \psi(x)\left[B-\int_{0}^{T_{0}} \varphi^{\prime}(t) u(t) d t\right](x) d x$ and $-\int_{\bar{\Omega}^{c}} \psi(x)[B-$ $\left.\int_{0}^{T_{0}} \varphi(t) \partial_{t} u(t) d t\right](x) d x$ coincide, where we used the abbreviation $B:=L^{q}\left(\bar{\Omega}^{c}\right)^{3}$. Thus $u \in$ $W^{1, q}\left(0, T_{0}, L^{q}\left(\bar{\Omega}^{c}\right)^{3}\right)$, and the derivative of $u$ as a function from $\left(0, T_{0}\right)$ into $L^{q}\left(\bar{\Omega}^{c}\right)^{3}$ coincides with the derivative $\partial_{t} u$ of $u$ as a function from $W_{q, T_{0}}$. It follows with [44, Lemma 3.1.1] that $u$ belongs to $C^{0}\left(\left[0, T_{0}\right], L^{q}\left(\bar{\Omega}^{c}\right)^{3}\right)$. Due to the relation $\nabla_{x} \pi \in L^{q}\left(0, T_{0}, L^{q}\left(\bar{\Omega}^{c}\right)^{3}\right)$, and by a transition from $\pi(t)$ to $\pi(t)-\left|\Omega_{R_{0}}\right|^{-1} \int_{\Omega_{R_{0}}} \pi(x, t) d x$, it may be supposed without loss of generality that $\pi \mid \Omega_{R_{0}} \times\left(0, T_{0}\right)$ belongs to $L^{q}\left(0, T_{0}, L^{q}\left(\Omega_{R_{0}}\right)\right)$. Also since $\nabla_{x} \pi \in L^{q}\left(0, T_{0}, L^{q}\left(\bar{\Omega}^{c}\right)^{3}\right)$, we obtain by [27, Theorem II.6.1] that (5.5) holds with $m_{0}=1, \gamma_{1}=q, \pi^{(1)}=\pi \mid{\overline{B_{R_{0}}}}^{c}$. Concerning the preceding reference to [27], we note that in the case $q \geq 3$, if $x_{0} \in \Omega, a \in(0, \infty)$ with $B_{a}\left(x_{0}\right) \subset \Omega$, and if $R_{0}$ is sufficiently large with respect to $\left|x_{0}\right|$ and $1 / a$, it may be achieved that $(1+|x|) \ln (2+|x|) \geq$ $\left(\left|x-x_{0}\right| \ln \left(\left|x-x_{0}\right| / a\right)\right) / 2 \geq\left|x-x_{0}\right| / 2$ for $x \in B_{R_{0}}^{c}$.

\section{References}

[1] Adams, R. A.; Fournier, J. J. F.: Sobolev spaces (2nd ed.). Academic Press, Amsterdam, 2003.

[2] Bae, H.-O., Jin, B. J.: Estimates of the wake for the 3D Oseen equations. Discrete Contin. Dyn. Syst. Ser. B 10 (2008), 1-18.

[3] Bae, H.-O., Roh, J.: Stability for the 3D Navier-Stokes equations with nonzero far field velocity on exterior domains. J. Math. Fluid Mech. 14 (2012), 117-139. 
[4] Borchers, W.; Sohr, H.: On the equations rot $v=g$ and $\operatorname{div} u=f$ with zero boundary conditions. Hokkaido Math. J. 19 (1990), 67-87.

[5] Deuring, P.: Exterior stationary Navier-Stokes flows in 3D with nonzero velocity at infinity: asymptotic behaviour of the velocity and its gradient. IASME Transactions 6 (2005), 900-904.

[6] Deuring, P.: The single-layer potential associated with the time-dependent Oseen system. In: Proceedings of the 2006 IASME/WSEAS International Conference on Continuum Mechanics. Chalkida, Greeece, May 11-13, 2006, 117-125.

[7] Deuring, P.: On volume potentials related to the time-dependent Oseen system. WSEAS Transactions on Math. 5 (2006), 252-259.

[8] Deuring, P.: On boundary driven time-dependent Oseen flows. Banach Center Publications 81 (2008), 119-132.

[9] Deuring, P.: A potential theoretic approach to the time-dependent Oseen system. In: Rannacher, R., Sequeira, A. (eds.): Advances in Mathematical Fluid Mechanics. Dedicated to Giovanni Paolo Galdi on the Occasion of his 60th Birthday. Springer, Berlin e. a., 2010, p. 191-214.

[10] Deuring, P.: Spatial decay of time-dependent Oseen flows. SIAM J. Math. Anal. 41 (2009), 886-922.

[11] Deuring, P.: A representation formula for the velocity part of 3D time-dependent Oseen flows. J. Math. Fluid Mech. 16 (2014), 1-39.

[12] Deuring, P.: The Cauchy problem for the homogeneous time-dependent Oseen system in $\mathbb{R}^{3}$ : spatial decay of the velocity. Math. Bohemica 138 (2013), 299-324.

[13] Deuring, P.: Pointwise spatial decay of time-dependent Oseen flows: the case of data with noncompact support. Discrete Contin. Dyn. Syst. Ser. A 33 (2013), 2757-2776.

[14] Deuring, P.: Spatial decay of time-dependent incompressible Navier-Stokes flows with nonzero velocity at infinity. SIAM J. Math. Anal. 45 (2013), 1388-1421.

[15] Deuring, P.: Pointwise spatial decay of weak solutions to the Navier-Stokes system in 3D exterior domains. J. Math. Fluid Mech. 17, 199-232 (2015).

[16] Deuring, P.: Pointwise decay in space and in time for incompressible flow around a rigid body moving with constant velocity. J. Math. Fluid Mech. 21, article 11 (2019) (35 pages).

[17] Deuring, P.: $L^{q}$-weak solutions to the time-dependent Oseen system: decay estimates. Submitted. Accessible at "https://hal.archives-ouvertes.fr/hal-02465651".

[18] Deuring, P.: Time-dependent incompressible viscous flows around a rigid body: estimates of spatial decay independent of boundary conditions. Submitted. Accessible at "https://hal.archives-ouvertes.fr/hal-02508815". 
[19] Deuring, P., Hishida, T.: Spatial asymptotics of mild solutions to the time-dependent Oseen system. In preparation.

[20] Deuring, P., Kračmar, S.: Exterior stationary Navier-Stokes flows in 3D with nonzero velocity at infinity: approximation by flows in bounded domains. Math. Nachr. 269-270 (2004), 86-115.

[21] Deuring, P., Kračmar, S., Nečasová, Š.: On pointwise decay of linearized stationary incompressible viscous flow around rotating and tranlating bodies. SIAM J. Math. Anal. 43 (2011), 705-738.

[22] Deuring, P., Varnhorn, W.: On Oseen resolvent estimates. Diff. Int. Equat. 23, 11391149 (2010).

[23] Enomoto, Y., Shibata, Y.: Local energy decay of solutions to the Oseen equation in the exterior domain. Indiana Univ. Math. J. 53 (2004), 1291-1330.

[24] Enomoto, Y., Shibata, Y.: On the rate of decay of the Oseen semigroup in exterior domains and its application to Navier-Stokes equation. J. Math. Fluid Mech. 7 (2005), 339-367.

[25] Farwig, R.: The stationary exterior 3D-problem of Oseen and Navier-Stokes equations in anisotropically weighted Sobolev spaces. Math. Z. 211 (1992), 409-447.

[26] Fučik, S., John, O., Kufner, A.: Function spaces. Noordhoff, Leyden 1977.

[27] Galdi, G. P.: An Introduction to the Mathematical Theory of the Navier-Stokes Equations. Steady-State Problems, 2nd edition. Springer, New York e.a., 2011.

[28] Heywood, J. G.: The exterior nonstationary problem for the Navier-Stokes equations. Acta Math. 129 (1972), 11-34.

[29] Heywood, J. G.: The Navier-Stokes equations. On the existence, regularity and decay of solutions. Indiana Univ. Math. J. 29 (1980), 639-681.

[30] Hille, E., Phillips, R. S.: Functional analysis and semi-groups. American Math. Soc. Colloquim Publicatons Vo.31, American Mathematical Society, Providence R. I., 1957.

[31] Knightly, G. H.: Some decay properties of solutions of the Navier-Stokes equations. In: Rautmann, R. (ed.): Approximation methods for Navier-Stokes problems. Lecture Notes in Math. 771, Springer, 1979, 287-298.

[32] Kobayashi, T., Shibata, Y.: On the Oseen equation in three-dimensional exterior domains. Math. Ann. 310 (1998), 1-45.

[33] Kračmar, S., Novotný, A., Pokorný, M.: Estimates of Oseen kernels in weighted $L^{p}$ spaces. J. Math. Soc. Japan 53 (2001), 59-111.

[34] Masuda, K.: On the stability of incompressible viscous fluid motions past bodies. J. Math. Soc. Japan 27 (1975), 294-327. 
[35] Miyakawa, T.: On nonstationary solutions of the Navier-Stokes equations in an exterior domain. Hiroshima Math. J. 12 (1982), 115-140.

[36] Mizumachi, R.: On the asymptotic behaviour of incompressible viscous fluid motions past bodies. J. Math. Soc. Japan 36 (1984), 497-522.

[37] Shen, Zongwei: Boundary value problems for parabolic Lamé systems and a nonstationary linearized system of Navier-Stokes equations in Lipschitz cylinders. American J. Math. 113 (1991), 293-373.

[38] Simader, C. G., Sohr, H.: The Dirichlet problem for the Laplacian in bounded and unbounded domains. Pitman Research Notes in Mathematics Vol. 321, CRC Press, London, 1996.

[39] Shibata, Y.: On an exterior initial boundary value problem for Navier-Stokes equations. Quarterly Appl. Math. 57 (1999), 117-155.

[40] Sohr, H.: The Navier-Stokes equations. An elementary functional analytic approach. Birkhäuser, Basel e.a., 2001.

[41] Solonnikov, V. A.: A priori estimates for second order parabolic equations. Trudy Mat. Inst. Steklov. 70 (1964), 133-212 (Russian); English translation, AMS Translations 65 (1967), 51-137.

[42] Solonnikov, V. A.: Estimates for solutions of nonstationary Navier-Stokes equations. Zap. Nauchn. Sem. Leningrad. Otdel. Mat. Inst. Steklov. (LOMI) 38 (1973), 153-231 (Russian); English translation, J. Soviet Math. 8 (1977), 467-529.

[43] Takahashi, S.: A weighted equation approach to decay rate estimates for the NavierStokes equations. Nonlinear Anal. 37 (1999), 751-789.

[44] Teman, R.: Navier-Stokes equations. Theory and numerical analysis. AMS Chelsea Publishing, Providence R.I., 2001.

[45] Weis, L.: Operator-valued Fourier multiplier theorems and maximal regularity. Math. Ann. 319 (2001), 735-758.

[46] Yoshida, K.: Functional analysis (6th ed.). Springer, Berlin e.a., 1980. 